\title{
Measurement of the Charge Asymmetry in Top-Antitop Quark Production WITH THE CDF II EXPERIMENT
}

JuLia WEINELT

\section{DIPLOMA THESIS}

AT The FACUlTy FOR Physics

OF THE UNIVERSITY OF KARLSRUHE (TH)

Referee: Prof. Dr. Th. Müller

Institut für Experimentelle Kernphysik

Co-Referee: Priv.-Doz. Dr. W. Wagner

Institut für Experimentelle Kernphysik

December 15, 2006 



\section{Introduction}

The Fermi National Laboratory (Fermilab) operates the Tevatron proton - antiproton collider at a center-of-mass energy of $\sqrt{s}=1.96 \mathrm{TeV}$, and is therefore the only collider which is today able to produce the heaviest known particle, the top quark.

The top quark was discovered at the Tevatron by the CDF and D $\varnothing$ collaborations in $1995[1,2]$. At the Tevatron, most top quarks are produced via the strong interaction, whereby quark-antiquark annihilation dominates with 85\%, and gluon fusion contributes with 15\%. Considering next-to-leading order (NLO) contributions in the cross section of top-antitop quark production, leads to a slight positive asymmetry in the differential distribution of the production angle $\alpha$ of the top quarks. This asymmetry is due to the interference of certain NLO contributions. The charge asymmetry $A$ in the cosine of $\alpha$ is predicted [14] to amount to $4-6 \%$. Information about the partonic rest frame, necessary for a measurement of $A$ in the observable $\cos \alpha$, is not accessible in the experiment. Thus, we use the rapidity difference of the top and the antitop quark as sensitive variable. This quantity offers the advantage of Lorentz invariance and is uniquely correlated with the cosine of $\alpha$, justifying the choice of the rapidity difference to describe the behaviour of $\cos \alpha$.

In preparation for a measurement of the charge asymmetry, we conduct several Monte Carlo based studies concerning the effect of different event selection criteria on the asymmetry in the selected event samples. We observe a strong dependence of the measured asymmetry on the number of required jets in the particular event sample. This motivates further studies to understand the influence of additional gluon radiation, which leads to more than four observed jets in an event, on the rapidity distribution of the produced top quarks. We find, that events containing hard gluon radiation are correlated with a strong negative shift of the rapidity distribution of the top quarks. This leads to large negative values of the charge asymmetry in event samples that contain only events with exactly five, six or more jets.

This finding requires a modification of our original analysis strategy, since an asymmetry measured in an inclusive sample will be a composition of the asymmetry in the four-jets and five-jets sub-samples. Therefore, we perform for the first time a measurement of the asymmetry separately in the exclusive four- and five-jets sub-samples to separate the contribution of hard gluon radiation to the asymmetry. We analyze a data sample, collected by the CDF II detector in the years 2002-2006, that corresponds to an integrated luminosity of about $955 \mathrm{pb}^{-1}$.

This thesis is organized as follows. After giving a brief overview of the theoretical backgrounds in the first chapter, the Tevatron and the CDF II experiment are 
described in the second chapter. The third chapter provides details about the event selection in the lepton + jets channel and outlines the reconstruction method of the four-vectors of the top-antitop quark pair. In chapter four, the event generation using Monte Carlo methods is described, and generators used in this thesis are shortly introduced. Some properties of the studied $t \bar{t}$ process at generator level are presented. The Monte Carlo based study of the asymmetry in top-pair production is presented in chapter five. In chapter six, the correction of the analyzed data for reconstruction effects and selection efficiencies in the inclusive and exclusive samples is described before providing the corresponding measurements. The results and an outlook can be found in the last chapter. 


\section{Contents}

1 Theory 7

1.1 The Standard Model of Elementary Particle Physics . . . . . . . . . . 7

1.2 The Top Quark . . . . . . . . . . . . . . . . 10

1.3 Charge Asymmetry in Top-Pair Production . . . . . . . . . . . 12

1.3.1 Sensitive Variable . . . . . . . . . . . . . . 15

2 The CDF Experiment $\quad 19$

2.1 The Accelerator System . . . . . . . . . . . . . . . 20

2.2 The Collider Detector at Fermilab . . . . . . . . . . . . . . . 23

2.2.1 Tracking System ................. 24

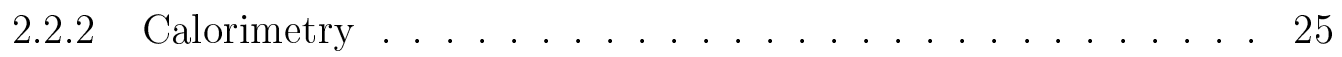

2.2 .3 Muon System . . . . . . . . . . . . . . . 26

2.3 The CDF Trigger System . . . . . . . . . . . . 26

3 Event Selection and Reconstruction $\quad 29$

3.1 Event Selection in the Lepton + Jets Channel . . . . . . . . . . . . . 29

3.2 Full Reconstruction of the Top Pair . . . . . . . . . . . . . . . . . 34

4 MC Study of Top-Pair Production 37

4.1 Monte Carlo Event Generators . . . . . . . . . . . . . . . . . 37

4.2 Top-Pair Production at Monte Carlo Level . . . . . . . . . . . . . . . 41

5 MC Study of Asymmetry in Top-Pair Production 47

5.1 Influence of the Event Selection on the Asymmetry . . . . . . . . . . 47

5.1.1 Influence of Different Cuts at Parton Level (PL) . . . . . . . . 49

5.1 .2 Influence of Different Cuts at Jet Level (JL) . . . . . . . . . 51

5.1.3 Influence of the CDF Standard Lepton + Jets Event Selection on $A\left(\Delta y \cdot Q_{l}\right) \ldots \ldots \ldots \ldots$. . . . . . . . . 56

5.2 Effect of Gluon Radiation on Top-Quark Rapidity Distributions . . . 60

5.2.1 Asymmetry $A\left(\Delta y \cdot Q_{l}\right)$ for Different $t \bar{t}$ Production Processes . 60

5.2.2 Study of the Event Generation with Prthia . . . . . . . . . . 61

5.2.3 Study of the Event Generation with MAdEvent . . . . . . 72

5.3 Summary . . . . . . . . . . . . . . 77 
6 Measurement of the Charge Asymmetry in Top-Pair Production 79

6.1 Background Estimation . . . . . . . . . . . . . . 79

6.2 Correction for Acceptance and Smearing Effects . . . . . . . . . . . . 81

6.3 Inclusive and Exclusive Measurement . . . . . . . . . . . . . . 85

6.4 Systematics . . . . . . . . . . . . . . . . 90

$\begin{array}{lll}7 & \text { Conclusion and Outlook } & 95\end{array}$

A Statistical Uncertainty of Corrected Event Numbers $\quad 97$ 


\section{List of Figures}

1.1 Feynman Diagram of $t \bar{t}$ pair production via quark-antiquark annihilation . . . . . . . . . . . . . . . . . . . 10

1.2 Leading order Feynman diagrams of $t \bar{t}$ pair production . . . . . . . 11

1.3 The CTEQ5M1 parton distribution function at $\mu=(175 \mathrm{GeV})^{2} \ldots 13$

1.4 LO and NLO diagrams for top-pair production via quark-antiquark

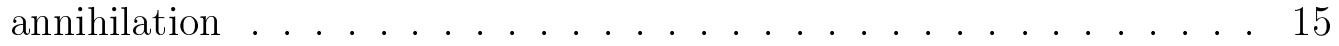

1.5 Charge asymmetry as a function of $\cos \alpha \ldots \ldots \ldots \ldots$

1.6 Difference of the rapidities as a function of $\cos \alpha$ and $\sqrt{\hat{s}} \ldots \ldots 17$

2.1 Aerial view on the Fermilab facility . . . . . . . . . . . . . 19

2.2 Schematic overview on the accelerator chain at Fermilab . . . . . . 21

2.3 The collider run II peak luminosity . . . . . . . . . . . . . . 22

2.4 Evolution of the extracted data amount at CDF . . . . . . . . 23

2.5 Cutaway view of the CDF II detector . . . . . . . . . . . . . . 24

2.6 Longitudinal view of the CDF II tracking system and the plug calorime-

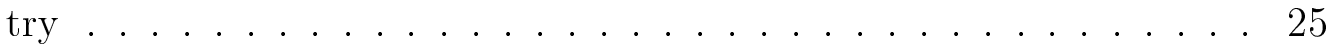

2.7 Functional block diagram of the CDF II data flow . . . . . . . . 27

3.1 Feynman diagram of top-pair production . . . . . . . . . . . 30

3.2 Schematic view of parameters used by the SecVtx algorithm . . . . 33

4.1 The evolution of QCD events . . . . . . . . . . . . 38

4.2 Monte Carlo generated production rates for top-quark pairs at the Tevatron, branching rates for the different possible decay channels and the $\sqrt{\hat{s}}$ distribution. . . . . . . . . . . . . . 43

4.3 Properties of the produced top-quark pair. . . . . . . . . . . . 44

4.4 Monte Carlo generated transverse momentum and pseudo-rapidity distributions of the charged leptons and neutrinos in top-pair events in the lepton+jets channel. . . . . . . . . . . . . . . 46

5.1 Monte Carlo distribution of $\Delta y \cdot Q_{l}$ at generator level . . . . . . 48

5.2 Distributions of $\Delta y \cdot Q_{l}$ for different cut applications on parton level . 50

5.3 Distributions of $\Delta y \cdot Q_{l}$ for different cut applications on hadron level 52

5.4 Asymmetry as a function of $N_{\text {jets }}$ for the MC@NLO sample with linear

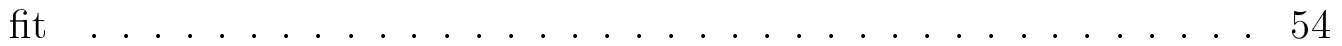

5.5 Asymmetry as a function of $N_{\text {jets }}$ for the MC@NLO sample if different cuts are applied . . . . . . . . . . . . . . . 55 
5.6 Asymmetry as a function of $N_{\text {jets }}$ for different jet clustering cut-offs . 56

5.7 Dependence of the asymmetry on the upper $\eta$-range cut-off . . . . . . 59

5.8 Asymmetry as a function of $N_{\text {jets }}$ for different top-pair production mechanism ..................... 60

5.9 Asymmetry as a function of $N_{\text {jets }}$ if different Monte Carlo processes are switched off . . . . . . . . . . . . . . 62

5.10 Asymmetry as a function of $N_{\text {jets }}$ if the Monte Carlo processes ISR and FSR are switched off .................. 63

5.11 Asymmetry as a function of $N_{\text {jets }}$ if the Monte Carlo process "multiple interactions" is switched off . . . . . . . . . . . . . . . 64

5.12 Illustration of color flow in $t \bar{t}$ events produced via quark-antiquark annihilation with gluon radiation . . . . . . . . . . . . 65

5.13 Transverse momentum of radiated gluons off the initial state . . . . . 67

5.14 Correlation between top (antitop) quark rapidity and transverse momentum $p_{T}^{I S R}$ of the corresponding radiation . . . . . . . . . . . 69

5.15 Correlation between top (antitop) quark rapidity and transverse momentum $\Delta p_{T}^{I S R}$ of the corresponding radiation . . . . . . . . . . 70

5.16 Correlation between top (antitop) quark rapidity and rapidity $y_{I S R}$ of the corresponding radiation . . . . . . . . . . . . . 71

5.17 Rapidity distributions of top and antitop quarks in $t \bar{t} j$ events generated by MAdEVEnT . . . . . . . . . . . . . . . 73

5.18 Transverse momentum of ISR/FSR objects generated by MADEvENT 74

5.19 Correlation between top (antitop) quark rapidity and transverse momentum of the corresponding ISR/FSR objects . . . . . . . . . 75

5.20 Correlation between top (antitop) quark rapidity and rapidity of the corresponding ISR/FSR objects . . . . . . . . . . . 76

6.1 Relative efficiencies of the $\Delta y \cdot Q_{l}$ distribution . . . . . . . . . . 82

6.2 Migration plots of $\Delta y \cdot Q_{l}$ for the inclusive PYTHiA sample as well as for the exclusive cases . . . . . . . . . . . . 83

6.3 Comparison of data with MC expectation plus background estimation 86

6.4 Comparison of asymmetry of the background subtracted event numbers with $\mathrm{MC}$ expectation in the exclusive four- and five-jets bins. . . 88

6.5 Statistical uncertainty $\sigma_{A}$ as a function of $N_{p} \ldots \ldots$. . . . . . 89

6.6 Comparison of measured exclusive results to the MC prediction . . . 90 


\section{List of Tables}

1.1 The elementary fermionic particles . . . . . . . . . . 8

1.2 The gauge bosons of the standard model . . . . . . . . . . 9

1.3 Decay modes of $t \bar{t}$ pairs and corresponding branching fractions . . . . 11

2.1 Design parameters of the CDF II muon detectors . . . . . . . . 26

3.1 Number of $t \bar{t}$ event candidates after performing the event selection for $955 \mathrm{pb}^{-1} \ldots \ldots \ldots \ldots \ldots \ldots \ldots \ldots$

4.1 Overview of used Monte Carlo samples in this analysis . . . . . . . 42

4.2 Branching fractions of the different top-pair decay channels . . . . . . 44

5.1 Monte Carlo values of the asymmetry at generator and reconstruction level . . . . . . . . . . . . . . . . . . 4 48

5.2 Effect of different selection cuts applied at parton level on the asym-

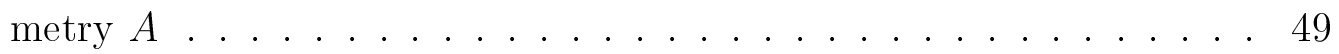

5.3 Effect of different selection cuts applied at hadron level on the asymmetry $A \ldots \ldots \ldots \ldots \ldots \ldots \ldots$

5.4 Asymmetry $A$ for different jet bins . . . . . . . . . . . . 53

5.5 Parameters of the linear fit functions of $A\left(N_{\text {jets }}\right) \ldots \ldots \ldots \ldots$

5.6 Asymmetry values if different groups of lepton + jets selection cuts are applied . . . . . . . . . . . . . . . . 5 58

5.7 Pythia Monte Carlo event record . . . . . . . . . . . . . . 66

5.8 MadEvent Monte Carlo event record . . . . . . . . . . . . . 72

6.1 Background estimation of different contributions in the inclusive and exclusive event samples. . . . . . . . . . . . . . . . . 80

6.2 Efficiencies $\epsilon_{p / n}$, smearing matrix elements $S(i, k)$, and correction matrix elements $C^{-1}(i, k)$ for the inclusive as well as for the exclusive analysis . . . . . . . . . . . . . . 85

6.3 Overview of number of $t \bar{t}$ events . . . . . . . . . . . . 87

6.4 Summary of systematic uncertainties . . . . . . . . . . . . . 92 


\section{Chapter 1}

\section{Theory}

The standard model of elementary particle physics is up to now the most successful theory of a unified description of the properties of the elementary particles and their interactions. In the following we give a short introduction to the particle content of the standard model and its main ideas. Subsequently, the characteristics of the heaviest quark, the top quark, are outlined and the theoretical framework of the charge asymmetry in top pair production, one of the main aspects of this thesis, is presented.

\subsection{The Standard Model of Elementary Particle Physics}

The standard model of particle physics is a theory which describes the strong, weak, and electromagnetic fundamental forces, as well as the fundamental particles that make up all matter. It is a quantum field theory and consistent with both quantum mechanics and special relativity $[3,4]$. To date, almost all experimental tests of the three forces described by the standard model have agreed with its predictions. However, the standard model is not a complete theory of fundamental interactions, primarily because it does not describe the gravitational force, which is formulated independently by general relativity.

The standard model contains both fermionic and bosonic fundamental particles. Fermions, the constituents of matter, are particles which possess half-integer spin and obey the Pauli exclusion principle, which states that no fermions can share the same quantum state at the same time. There are twelve different types of fermions in the standard model, which can be arranged in three generations. In addition, the standard model predicts for each particle an antiparticle, which has the same properties but opposite charges. Table 1.1 presents the twelve fundamental particles with their main properties. The fermions are again partitioned in two classes of particles, the leptons and quarks. Unlike leptons, the quarks are characterized by an additional quantum number, the color charge, which is responsible for the strong coupling. Leptons, however, only participate in electromagnetic interactions (electrically charged leptons) and weak interactions. All ordinary matter is made 


\begin{tabular}{c|c|c|c}
\multicolumn{4}{c}{ Elementary fermionic particles } \\
name & symbol & el. charge $[e]$ & mass $\left[\mathrm{MeV} / c^{2}\right]$ \\
\hline \hline 1. Generation: & & & \\
electron & $e$ & -1 & 0.51 \\
electron neutrino & $\nu_{e}$ & 0 & $\leq 2 \cdot 10^{-6}$ \\
up quark & $u$ & $+\frac{2}{3}$ & $1.5 \ldots 3.0$ \\
down quark & $d$ & $-\frac{1}{3}$ & $3 \ldots 7$ \\
\hline 2. Generation: & & & 106 \\
muon & $\mu$ & -1 & $\leq 0.190$ \\
muon neutrino & $\nu_{\mu}$ & 0 & $70 \ldots 120$ \\
charm quark & $c$ & $+\frac{2}{3}$ & $(1.16 \ldots 1.34) \cdot 10^{3}$ \\
strange quark & $s$ & $-\frac{1}{3}$ & 1777 \\
\hline 3. Generation: & & & $\leq 18.2$ \\
tau & $\tau$ & -1 & $(170.2 \ldots 174.8) \cdot 10^{3}$ \\
tau neutrino & $\nu_{\tau}$ & 0 & $(4.13 \ldots 4.27) \cdot 10^{3}$ \\
top quark & $t$ & $+\frac{2}{3}$ & $-\frac{1}{3}$ \\
bottom quark & $b$ & \multicolumn{2}{c}{} \\
\hline \hline
\end{tabular}

Table 1.1: The elementary fermionic particles ( $\operatorname{spin} s=\frac{1}{2}$ ) with their electric charge in units of the electron charge and their mass [5].

of first-generation particles (up quark, down quark, electron and electron neutrino). The higher-generation particles decay quickly into the first-generation ones and can only be observed in high-energy interactions as they occur in particle experiments, in the cosmos or in the atmosphere. The reason for arranging them in generations is that the four fermions in each generation behave almost exactly like their counterparts in the other generations, the only difference is in their masses.

The standard model includes the strong, the weak and the electromagnetic forces that are mathematically described by gauge theories. This means that the forces between fermions are modeled by coupling them to bosons, integer spin particles which mediate the forces. The Lagrangian, a fundamental mathematical concept describing the system, is required to be invariant under a transformation called gauge transformation. The invariance of the Lagrangian is only possible if additional fields are introduced. The quanta of these fields are referred to as gauge bosons, mediating the different forces. The bosons in the standard model and some of their properties are listed in table 1.2. The photon, a massless electric neutral boson, mediates the electromagnetic force by coupling to all particles that are electrically charged. Quantum Electro Dynamics (QED) provides a theoretical description of the electromagnetic interactions by the exchange of photons. Quantum Chromo Dynamics (QCD) describes strong interactions via gluons. Since the strong coupling is due to the color charge, only quarks and the mediating gluons, which are also color charged particles, participate in strong interactions. The fact that gluons carry color themselves leads to a phenomenon called confinement that interdicts the observation of free quarks since they are immediately bound to colorless bosonic mesons (quark- 
antiquark pairs) or fermionic baryons (quark triplets) by the exchange of gluons. The weak interaction was successfully unified with the electromagnetic theory in the electroweak theory in the middle sixties by Glashow, Salam and Weinberg and has been extensively tested during the last 40 years. The weak force couples to the weak isospin and the hypercharge of particles and is mediated by the massive neutral $Z$ boson and the electrically charged $W$ bosons (see table 1.2). The weak force plays an important role for particle decays, since it provides the only way to change the particle flavor. Considering quark flavor changing currents one has to take care of the fact that the quark mass eigenstates $(u, d, s, c, t, b)$ are not equal to the weak eigenstates of the quarks $\left(u^{\prime}, d^{\prime}, s^{\prime}, c^{\prime}, t^{\prime}, b^{\prime}\right)$. A transformation between the two bases is described by the Cabbibo-Kobayashi-Maskawa matrix (CKM matrix) [6,7]. By convention, the matrix is often expressed in terms of a $3 \times 3$ unitary matrix $V$ operating on the quark mass eigenstates $(d, s$ and $b)$ :

$$
\left(\begin{array}{c}
d^{\prime} \\
s^{\prime} \\
b^{\prime}
\end{array}\right)=\left(\begin{array}{lll}
V_{u d} & V_{u s} & V_{u b} \\
V_{c d} & V_{c s} & V_{c b} \\
V_{t d} & V_{t s} & V_{t b}
\end{array}\right) \cdot\left(\begin{array}{c}
d \\
s \\
b
\end{array}\right)
$$

The coupling strength of two quarks $i$ and $k$ to a $W$ boson is proportional to the according squared element $\left|V_{i k}\right|^{2}$ of the CKM matrix. The values of individual matrix elements can in principle all be determined from weak decays of the relevant quark, or, in some cases, from deep inelastic neutrino scattering. The $90 \%$ confidence limits on the magnitude of the elements of the complex matrix are [5]:

$$
\left(\begin{array}{lll}
0.97360 \ldots 0.97407 & 0.2262 \ldots 0.2282 & 0.00306 \ldots 0.00486 \\
0.2261 \ldots 0.2281 & 0.97272 \ldots 0.97320 & 0.04141 \ldots 0.04231 \\
0.00750 \ldots 0.00846 & 0.04083 \ldots 0.04173 & 0.999096 \ldots 0.999134
\end{array}\right)
$$

Since particle physics happens in the quantum mechanical regime, it is impossible to predict individual events. The theory only provides transition rates in the form of cross sections, a measurement for the probability that a certain process occurs. The cross section of a physical process is calculated by use of Fermi's Golden Rule [8], which states that the cross section is proportional to the square of the sum of contributing matrix elements of a certain process. The contributions to a matrix

\begin{tabular}{c|c|c|c|c} 
name & symbol & mediated force & electric charge $[e]$ & mass $\left[\mathrm{MeV} / c^{2}\right]$ \\
\hline \hline photon & $\gamma$ & electromagnetic & 0 & 0 \\
gluon & $g$ & strong & 0 & 0 \\
$Z$ boson & $Z^{0}$ & weak & 0 & $91.188 \pm 0.002$ \\
$W$ bosons & $W^{ \pm}$ & weak & \pm 1 & $80.403 \pm 0.029$ \\
\hline \hline
\end{tabular}

Table 1.2: The gauge bosons mediating different forces of the standard model. The electric charge and the mass of the bosons are shown [5]. 
element are derived from Feynman diagrams, symbolic notations of physical interaction between particles. Following the Feynman rules, each part of such a diagram can be translated to a mathematical expression that enters the calculation of the matrix element. The lines represent the propagation of particles and antiparticles in space-time, the nodes connecting the particles contain the coupling nature and strength. Figure 1.1 shows the most elementary Feynman diagram for $t \bar{t}$ pair production, the Born diagram. By convention the $x$-axis represents the time coordinate of the particles and the $y$-axis the space coordinates. In figure 1.1 a quark and an antiquark annihilate to a virtual gluon, which again splits into a top-antitop pair.

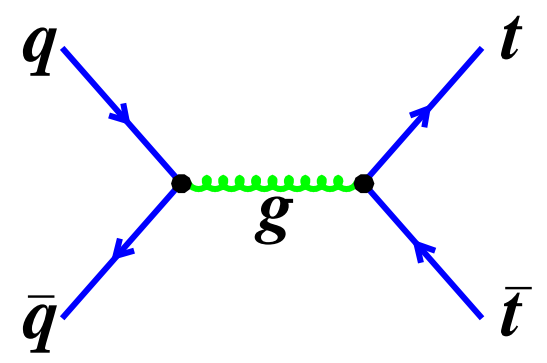

Figure 1.1: Feynman Diagram of $t \bar{t}$ pair production via quark-antiquark annihilation.

One of the main open questions concerning the standard model is the origin of the mass of elementary fermions and the $W^{ \pm}$and $Z$ bosons. Since the Lagrangian of the different quantum field theories is gauge invariant, the standard model allows no mass for the gauge bosons and therefore has to be extended by an additional mechanism. The most promising approach is the Higgs mechanism [9]. This theory introduces a scalar quantum field, the Higgs field, and ascribes the mass of the gauge bosons to an interaction between the field and the particles. The quantum of the scalar Higgs field is the massive Higgs boson, which has not yet been observed, but is expected to be found by next-generation hadron collider experiments at the Large Hadron Collider (LHC) at CERN.

\subsection{The Top Quark}

The top quark is a third-generation quark with a charge of $+\frac{2}{3} e$ and was discovered in 1995 by the CDF and $\mathrm{D} \varnothing$ experiments $[1,2]$. With a mass currently measured at $(172.5 \pm 2.3) \mathrm{GeV} / c^{2}[10]$ it is the most massive known particle.

At the Tevatron, where protons $(p)$ and antiprotons $(\bar{p})$ are brought to collision with a center-of-mass energy of $\sqrt{s}=1.96 \mathrm{TeV}$, most top quarks are pair-produced via the strong interaction. The dominant production process for $t \bar{t}$ pairs at the Tevatron is the quark-antiquark annihilation with an expected fraction of $85 \%$, while gluon fusion contributes with 15\%. The corresponding Feynman diagrams for the two dominant leading-order processes are shown in figure 1.2. The latest cross section calculation of $t \bar{t}$ pair production at a center-of-mass energy of $\sqrt{s}=1.96$ $\mathrm{TeV}$ and an assumed top quark mass of $m_{t}=175 \mathrm{GeV}$ is $\sigma_{t \bar{t}}=6.7 \pm 0.9 \mathrm{pb}$ [11]. The standard model predicts a top quark lifetime of about $10^{-25} \mathrm{~s}$ [5] which is less 


\begin{tabular}{c|ccc}
$\mathrm{W}$ decays & $e / \mu \nu$ & $\tau \nu$ & $q_{1} \bar{q}_{2}$ \\
\hline$e / \mu \nu$ & $4 / 81$ & $4 / 81$ & $\mathbf{2 4 / 8 1}$ \\
$\tau \nu$ & - & $1 / 81$ & $12 / 81$ \\
$q_{1} \bar{q}_{2}$ & - & - & $36 / 81$ \\
\hline \hline
\end{tabular}

Table 1.3: Decay modes of $t \bar{t}$ pairs. Presented are the branching fractions of leptonically and hadronically decaying $W$ bosons [12]. The $\tau$ channel is listed separately since the $\tau$ lepton decays before it can be observed in the experiment. The bold branching fraction indicates the channel used in this analysis.

than the time scale of the strong interaction and therefore the top quark decays before it is bound by the strong force to a colorless object. This gives us the unique opportunity of studying a bare quark by observing its decay particles on which the top quark transmitted its properties. Due to the dominating matrix element $\left|V_{t b}\right| \approx 1$ of the CKM matrix the top quark decays to almost $100 \%$ via the weak force into a $b$ quark and a $W$ boson. The $b$ quark hadronizes to a colorless object, which further decays and produces a range of secondary particles. The $W$ boson decays via the weak interaction, either hadronically into two light quarks or leptonically into a lepton $(e, \mu, \tau)$ and the corresponding neutrino $\left(\nu_{e}, \nu_{\mu}, \nu_{\tau}\right)$. Considering all lepton and quark flavors, 81 possibilities to decay arise for a produced top-antitop quark pair, each with a certain possibility to occur. Table 1.3 gives an overview of the different decay channels and the according branching ratio.

Each decay channel offers advantages and disadvantages which have to be weighted against each other with regard to the physical measurement. In our analysis we look into the channel, where one $W$ boson decays hadronically and the other one leptonically, the corresponding branching fraction of about $30 \%$ is bold in figure 1.3. The characteristics of this channel are outlined in chapter 3.

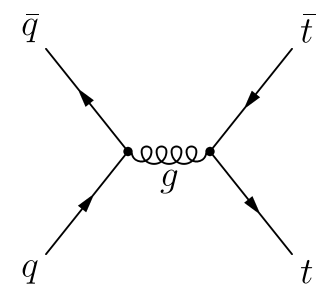

(a)

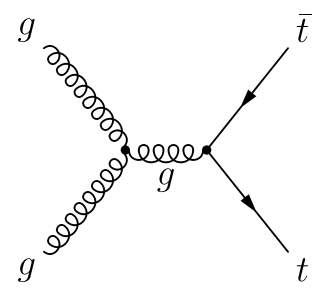

(b)

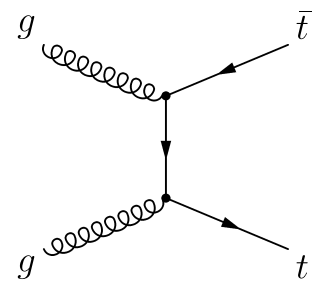

(c)

Figure 1.2: Leading order Feynman diagrams of $t \bar{t}$ pair production: (a) light quark-antiquark annihilation $(q \bar{q} \rightarrow t \bar{t})$, and gluon fusion $(g g \rightarrow t \bar{t})(\mathrm{b}),(\mathrm{c})$. 


\subsection{Charge Asymmetry in Top-Pair Production}

Since in nature complex processes occur as long as they are not explicitly interdicted, theoretical predictions must include also higher order calculations to be comparable to experimental results. The cross section of a certain physical scattering process $A B \rightarrow C D$ as observed by the experiment is determined by the contributions of different sub-processes. Sorting these subprocesses with respect to the order of the corresponding coupling constant $\alpha$ allows for a mathematical description of the total process by a perturbation series evolved in $\alpha$. A valid perturbative description is possible, if the coupling constant is clearly smaller than one, causing the expansion to converge. In this case, higher orders of the perturbation expansion are suppressed. Thus, the cross section can in general be approximated sufficiently by considering only the leading terms. In the case of QCD processes, the strong coupling constant $\alpha_{s}$ depends on the momentum transfer scale $Q^{2}$ of the process. In the limit of high energies $\alpha_{s}$ approaches zero. For the production of top quarks at the threshold of $m_{t}=175 \mathrm{GeV} / \mathrm{c}^{2}$ the requirement for convergence is well satisfied. The larger the coupling constant becomes, the more contributing terms of the expansion must be considered to receive a stable result. In return that means, that the more Feynman diagrams are taken into account, the more complicated becomes the calculation of the cross section. Since the mathematical method fails for the description of processes in the low energy limit, phenomenological models have to be used to model long distance QCD processes.

Theoretical calculations of cross sections at the Tevatron have to take into account that the incoming protons and antiprotons are not elementary particles since they are composed of a certain quark flavor combination. In the standard model of elementary particle physics the proton is described to be composed of two $u$ quarks and a $d$ quark (uud), the antiproton of the charge conjugated content $(\bar{u} \bar{u} \bar{d})$. These valence quarks are bound together by gluons that can split up into quarkantiquark pairs, the seaquarks. The parton model considers the high-energy proton (or antiproton) as a composition of these quasi-free partons: the valence quarks, the seaquarks and the gluons. Thus, the longitudinal momentum of the incoming proton (antiproton) is shared by those partons $i$, each carrying the momentum fraction $x_{i}=p_{i} / p_{p}$. The cross section calculation is based on the factorization theorem [12] stating that the cross section is given by the convolution of parton distribution functions (PDFs) $f_{i}\left(x, \mu^{2}\right)$ for the colliding proton $(p)$ and antiproton $(\bar{p})$ and the hard parton-parton cross section $\hat{\sigma}_{i j}$ :

$$
\sigma(p \bar{p} \rightarrow t \bar{t})=\sum_{i, j} \int d x_{i} d x_{j} f_{i, p}\left(x_{i}, \mu^{2}\right) f_{j, \bar{p}}\left(x_{j}, \mu^{2}\right) \cdot \hat{\sigma}_{i j}\left(i j \rightarrow t \bar{t} ; \hat{s}, \mu^{2}\right) .
$$

The PDF $f_{i, p}\left(x_{i}, \mu^{2}\right)$ describes the probability density to find a parton $i$ inside the proton $p$ carrying a longitudinal momentum fraction $x_{i}$. The PDFs and the partonparton cross section $\hat{\sigma}_{i j}$ depend on the factorization scale $\mu$. Since the production of top quarks is considered, a choice of the scale could be the top quark mass. Figure 1.3 shows the parton distribution function CTEQ5M1 [13] for protons at a 
factorization scale $\mu^{2}=(175 \mathrm{GeV})^{2}$. The parton cross section also depends on the square of the center-of-mass energy of the colliding partons: $\hat{s}=\left(x_{i} \cdot p_{p}+x_{j} \cdot p_{\bar{p}}\right)^{2}$. Since the proton and the antiproton beam have the same energy $E_{b e a m}, \hat{s}$ is given by: $\hat{s}=4 x_{i} x_{j} E_{\text {beam }}^{2}$.

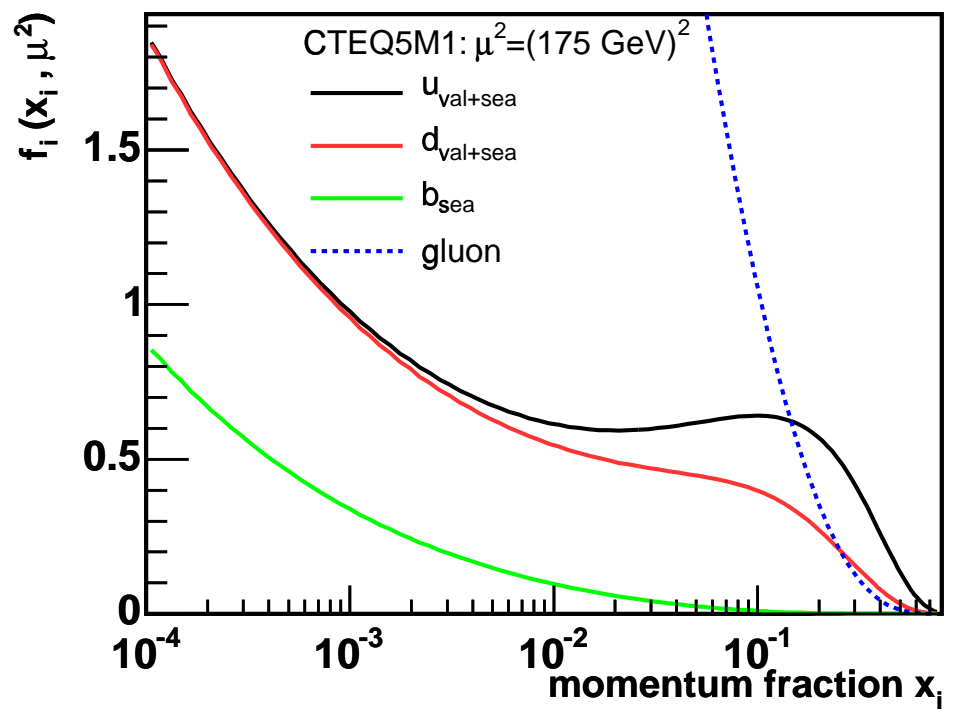

Figure 1.3: The CTEQ5M1 parton distribution function at a factorization scale $\mu^{2}=(175 \mathrm{GeV})^{2}$.

Although higher-order contributions to a cross section are suppressed due to the precondition of a small perturbation term, new phenomena arise only at nextto-leading (NLO) order level. In the following we describe the manifestation of the charge asymmetry in top pair production, a next-to-leading order interference effect predicted by J.H. Kühn [14]. The total asymmetry $A$ is described by the difference between the NLO cross section of top pair production via quark-antiquark annihilation compared to the NLO cross section of the charged conjugated final state:

$$
A=\frac{\sigma_{q \bar{q} \rightarrow t \bar{t}}^{N L O}(\cos \alpha>0)-\sigma_{q \bar{q} \rightarrow t t}^{N L O}(\cos \alpha>0)}{\sigma_{q \bar{q} \rightarrow t \bar{t}}^{L O}(\cos \alpha>0)+\sigma_{q \bar{q} \rightarrow \bar{t} t}^{L O}(\cos \alpha>0)} .
$$

The production angle $\alpha$ of the top quark is defined as the angle between the directions of the incoming quark and the produced top quark. The differential charge asymmetry as a function of the cosine of $\alpha$ is defined by [14] as:

$$
A(\cos \alpha)=\frac{N_{t}(\cos \alpha)-N_{\bar{t}}(\cos \alpha)}{N_{t}(\cos \alpha)+N_{\bar{t}}(\cos \alpha)}=\frac{N_{t}(\cos \alpha)-N_{t}(-\cos \alpha)}{N_{t}(\cos \alpha)+N_{t}(-\cos \alpha)} .
$$

The angular distribution of expected events $N(\cos \alpha)$ is derived from the differential cross section $\mathrm{d} \sigma / \mathrm{d} \cos \alpha$. Because the strong interaction is invariant under charge conjugation of the total process:

$$
\sigma_{q \bar{q} \rightarrow t \bar{t}}(\alpha)=\sigma_{\bar{q} q \rightarrow \overline{t t}}\left(180^{\circ}-\alpha\right)=\sigma_{\bar{q} q \rightarrow \bar{t} t}(-\alpha),
$$


$N_{\bar{t}}(\cos \alpha)=N_{t}(-\cos \alpha)$ holds and the charge asymmetry can be interpreted as a forward-backward asymmetry.

At leading-order perturbation theory only Feynman diagrams shown in figure 1.2 contribute to the matrix element, whereby the quark annihilation $q \bar{q} \rightarrow t \bar{t}$ dominates (see section 1.2). The corresponding differential cross sections for both quarkantiquark annihilation $q \bar{q} \rightarrow t \bar{t}$ and gluon-gluon fusion $g g \rightarrow t \bar{t}$ are symmetric in $\cos \alpha$, thus no asymmetry occurs.

Since gluon fusion remains symmetric under inclusion of higher-order contributions to $t \bar{t}$ pair production, figure 1.4 only shows the considered Feynman diagrams for quark-antiquark annihilation: the LO Born diagram, the box diagram, initial state gluon radiation (ISR), and final state gluon radiation (FSR). In the case of ISR/FSR, the process is only taken into account up to a cut-off of the transverse energy of the radiation. The cut-off allows only unresolvable collinear gluon radiation to count as a " $t \bar{t} "$ final state. If gluon radiation is hard and leads to an additional detectable jet, the final state is $t \bar{t} j$. The NLO cross section for $q \bar{q} \rightarrow t \bar{t}$ contains all amplitudes that are of third order in the strong coupling constant $\alpha_{s}$ and the leading order contributions $\left(\mathcal{O}\left(\alpha_{s}^{2}\right)\right)$, and is therewith proportional to:

$\sigma_{q \bar{q} \rightarrow t \bar{t}}^{N L O} \propto\left|\mathcal{M}_{L O}\right|^{2}+\left|\mathcal{M}_{I S R}\right|^{2}+\left|\mathcal{M}_{F S R}\right|^{2}+2 \cdot \Re\left(\mathcal{M}_{L O} \cdot \overline{\mathcal{M}}_{b o x}\right)+2 \cdot \Re\left(\mathcal{M}_{I S R} \cdot \overline{\mathcal{M}}_{F S R}\right)$

Thus the finally calculated term will not only contain the square of single matrix elements but also products of different complex amplitudes which are considered as interference terms.

The origin of the asymmetry can be explained qualitatively by considering the charge parity of the final states of the different sub-processes. Charge parity is a multiplicative quantum number describing the behavior of a physical system under the charge conjugation, which means that particles are substituted by their antiparticles. Since the gluon holds a negative charge parity $C=-1$ and $C$ is a conserved multiplicative quantum number in strong interactions, the charge parity of the produced $t \bar{t}$ pair in the Born diagram has to be $C(t \bar{t})=-1=C(g)$, see figure $1.4 \mathrm{a})$. The same argument holds for initial state gluon radiation (fig. $1.4 \mathrm{c})$ ). The box diagram (fig. $1.4 \mathrm{~b})$ ) containing two gluons requires $C(t \bar{t})=+1=C(g g)$. In case of final state gluon radiation (fig. $1.4 \mathrm{~d}$ )) the charge parity of the intermediated gluon has to be equal to $C$ of all final state particles, which leads to $C=+1$ for the top pair. Thus the terms, which enter quadratically in the cross section, are always positive under charge conjugation $(C=+1)$, while the interference terms, $\mathcal{M}_{L O} \cdot \overline{\mathcal{M}}_{\text {box }}$ and $\mathcal{M}_{I S R} \cdot \overline{\mathcal{M}}_{F S R}$, are both negative under charge conjugation $(C=-1)$. This leads to the fact that only odd amplitudes, i.e. the interference terms, remain in the difference in the numerator of equation 1.4, whereby terms with positive charge parity cancel each other. Consideration of the color charge of gluons complicates the basis of our illustration. Nevertheless, the qualitative argumentation remains.

The interference of the box diagram with the LO diagram yields a positive total asymmetry, while the interference between the ISR and FSR diagram leads to a negative total asymmetry. Because the former contribution is always larger than the latter one, the inclusive total asymmetry is slightly positive. Calculating the 
(a)

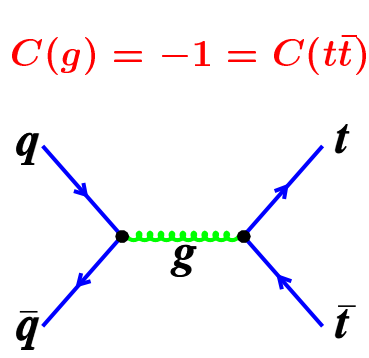

(b)

$C(g g)=1=C(t \bar{t})$

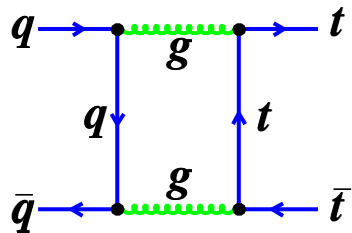

(c)

$C(g)=-1=C(t \bar{t})$



(d)

$C(g)=-1=C(t \bar{t} g)$

$\rightarrow C(t \bar{t})=1$

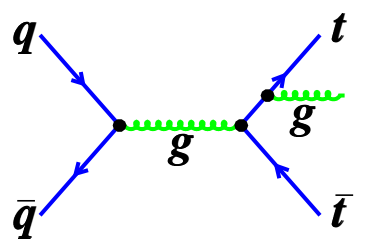

Figure 1.4: LO and NLO diagrams for top-pair production via quark-antiquark annihilation. (a) LO diagram, (b) box diagram, (c) ISR diagram, (d) FSR diagram.

total asymmetry with respect to the LO cross section as done in equation 1.4 yields a prediction of the differential asymmetry of $6-8 \%$ in $\cos \alpha$. If the asymmetry is calculated with respect to the NLO cross section, which is closer to an asymmetry measured by an experiment, since data always represent all order processes, the differential asymmetry is reduced by a factor of 1.3 to $4-6 \%$.

As presented in figure 1.5 the asymmetry depends linearly on the cosine of $\alpha$ for $\sqrt{\hat{s}}=400 \mathrm{GeV}$. Modifications from this linear behaviour increase with $\sqrt{\hat{s}}$ and in the limit of very large $\sqrt{\hat{s}}$ the same curve as for $b$ quarks is expected. Because $\sigma_{q \bar{q} \rightarrow t \bar{t}}(\sqrt{\hat{s}})$ peaks at values arround $\sqrt{\hat{s}} \sim 450 \mathrm{GeV}$ and because smaller values of $\sqrt{\hat{s}}$ are preferred due to the PDFs, the asymmetry is expected to depend almost linearly on $\cos \alpha$ at the Tevatron.

\subsubsection{Sensitive Variable}

The asymmetry is given in reference [15] as a function of $\cos \alpha$, but a measurement of the variable $\cos \alpha$ requires the reconstruction of the initial parton rest frame which is not accessible in the experiment. Therefore, we use a different quantity as sensitive variable in this analysis. Instead of $\cos \alpha$ the rapidity difference of the top quark $y_{t}$ and the antitop quark $y_{\bar{t}}$ is considered, whereby the rapidity of a particle is defined as:

$$
y=\frac{1}{2} \cdot \ln \left(\frac{E+p_{z}}{E-p_{z}}\right)
$$

This quantity transforms under a boost to a frame with velocity $\beta$ like $y \rightarrow y-$ $\tanh ^{-1} \beta$. Hence the shape of the rapidity distribution $\mathrm{dN} / \mathrm{dy}$ remains Lorentz invariant and is therefore independent of the particular physical frame. In our analysis $\Delta y$ is calculated by the rapidity difference of the semileptonically ( $\left.y_{\text {tlep }}\right)$ and the hadronically $\left(y_{\text {thad }}\right)$ decaying top quark. Multiplication with the charge of the lepton $(e$ or $\mu$ ) from the semileptonic top quark decay ensures that we always consider the rapidity difference between the top and the antitop quark:

$$
\Delta y \cdot Q_{l}=\left(y_{\text {tlep }}-y_{\text {thad }}\right) \cdot Q_{l}=y_{t}-y_{\bar{t}}
$$




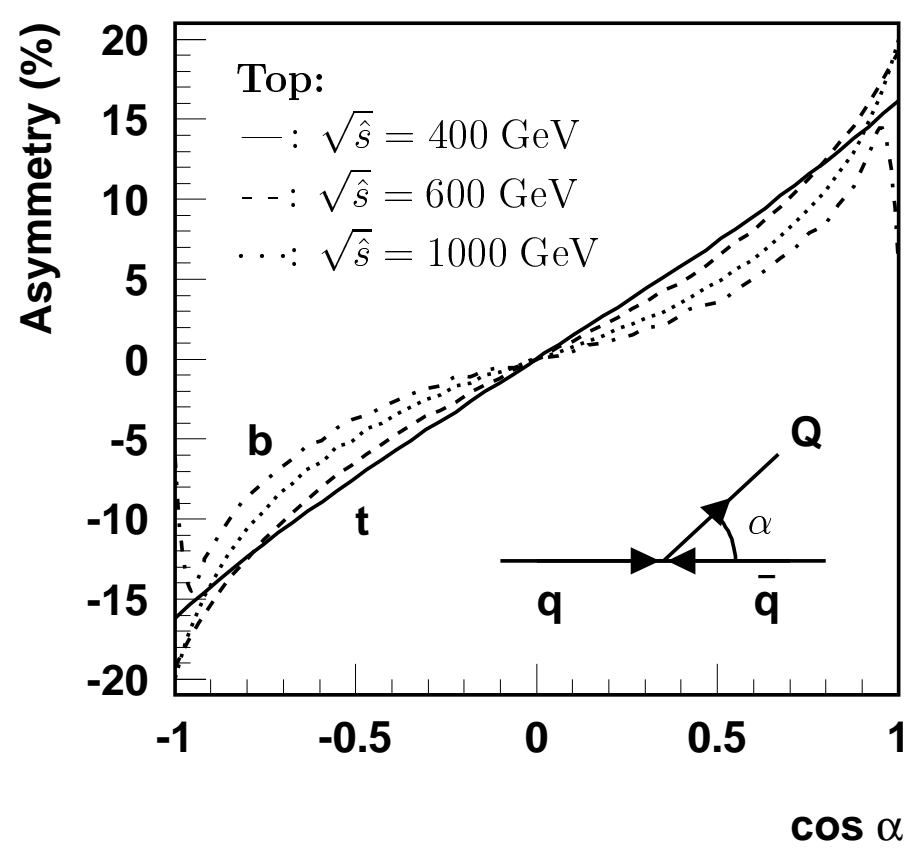

Figure 1.5: Charge asymmetry (eq. 1.5) as a function of $\cos \alpha$ for some fixed values of $\hat{s}$, reproduced from reference [15].

In LO $y_{t}-y_{\bar{t}}$ is directly related to the variable $\cos \alpha$ in the center-of-mass system [16]:

$$
y_{t}-y_{\bar{t}}=2 \cdot \tanh ^{-1}\left(\frac{\cos \alpha}{\sqrt{1+\frac{4 m_{t}^{2}}{\hat{s}-4 m_{t}^{2}}}}\right) .
$$

As can be seen from figure 1.6 there is no sign inversion between $\cos \alpha$ and $y_{t}-$ $y_{\bar{t}}$ for all values of $\sqrt{\hat{s}}$, which justifies the assumption that a measurement of the asymmetry in the observable $\Delta y \cdot Q_{l}$ represents the asymmetry in $\cos \alpha$ very well. The asymmetry in $\Delta y \cdot Q_{l}$ is then calculated analogously to equation 1.5 as the difference of events with a rapidity difference of the produced top quarks greater and smaller than zero divided by the total number of events:

$$
A\left(\Delta y \cdot Q_{l}\right)=\frac{N_{\mathrm{evts}}\left(\Delta y \cdot Q_{l}>0\right)-N_{\mathrm{evts}}\left(\Delta y \cdot Q_{l}<0\right)}{N_{\mathrm{evts}}\left(\Delta y \cdot Q_{l}>0\right)+N_{\mathrm{evts}}\left(\Delta y \cdot Q_{l}<0\right)}=\frac{N_{p}-N_{n}}{N_{p}+N_{n}},
$$

whereby the number of events with a positive (negative) rapidity difference is defined as $N_{p}\left(N_{n}\right)$. 


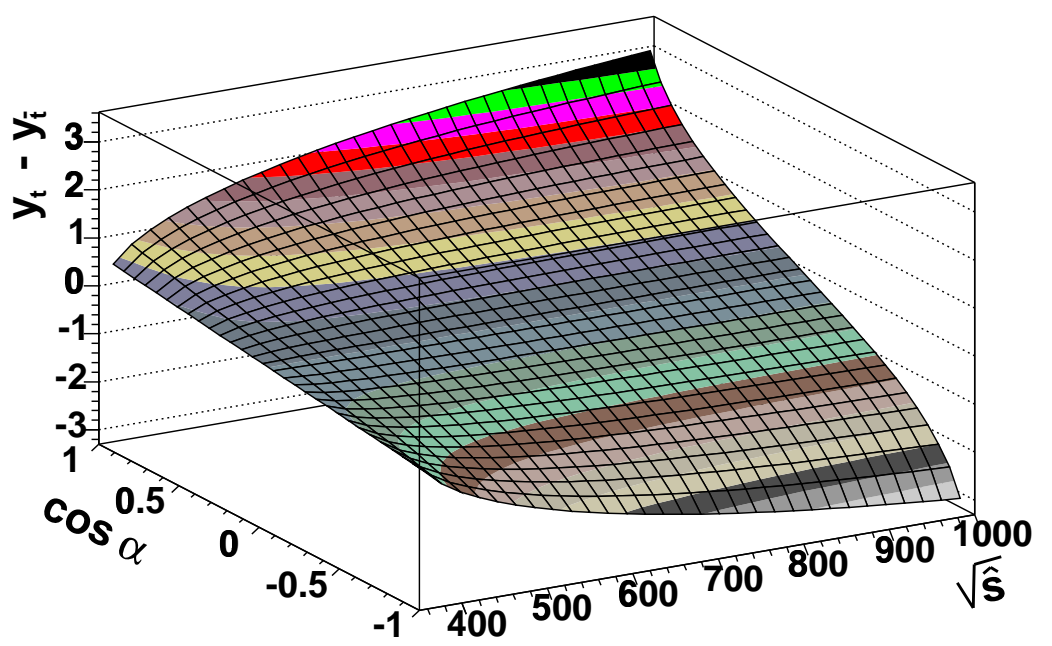

Figure 1.6: Difference of the rapidities of the top and the antitop quark as a function of $\cos \alpha$ and $\sqrt{\hat{s}}$. The correlation between $y_{t}-y_{\bar{t}}$ and $\cos \alpha$ remains unique in the displayed energy range of $400 \leq \sqrt{\hat{s}} \leq 1000 \mathrm{GeV}$, since there is no sign inversion. 


\section{Chapter 2}

\section{The CDF Experiment}

The Fermi National Accelerator Center (Fermilab) is located approximately $70 \mathrm{~km}$ west of downtown Chicago in Batavia, Illinois, in the United States of America. It operates the most powerful particle accelerator in the world at this time, providing high-energy proton and antiproton beams of $980 \mathrm{GeV}$. More than 2300 scientists from 34 states and 28 countries profit from Fermilab's equipment and particle beams to conduct a range of experiments. The accelerator concept offers the possibility for collider physics with a center-of-mass energy of $1.96 \mathrm{TeV}$ as well as to conduct fix target experiments.

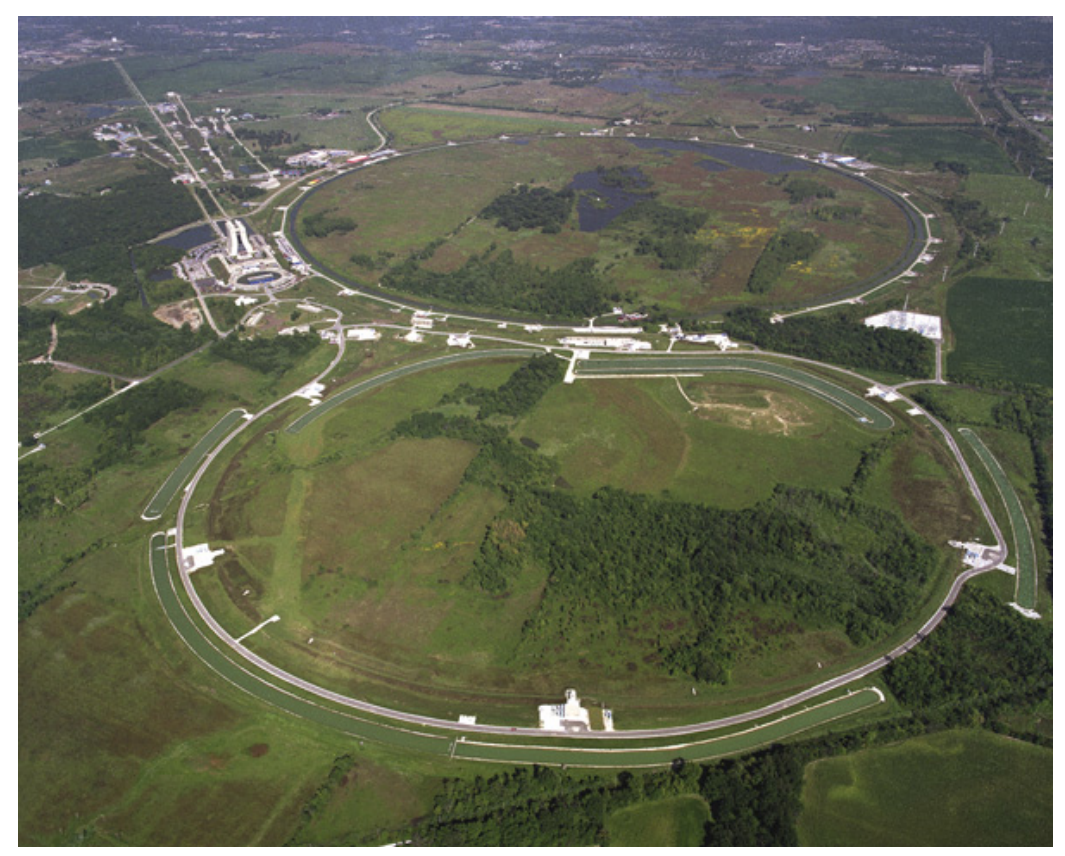

Figure 2.1: Aerial view on the Fermilab facility. The position of the Tevatron is indicated by the circular course of the rear maintenance road. The CDF experiment is located at the ten o'clock position at the Tevatron.

As can be seen from figure 2.1 presenting an aerial view of the large Fermilab facility various accelerator levels are necessary to reach such high particle energies. 
The circular maintenance roads indicate the position of the two largest Fermilab synchrotrons, the Main Injector in the foreground and the Tevatron, the final storage ring, in the rear. One of the two collider experiments at Fermilab is conducted by the Collider Detector at Fermilab (CDF) collaboration in which the Institut für Experimentelle Kernphysik Karlsruhe (IEKP) participates as the single German physics institute. The CDF experiment is located in figure 2.1 at the ten o'clock position at the Tevatron.

In the following section an overview of the complex accelerator chain is presented. Afterwards we give an introduction to the main components of the CDF II detector including an outline of the ambitious trigger system which is essential to extract the most interesting signals from the full range of detector responses.

\subsection{The Accelerator System}

To accomplish an acceleration of protons and antiprotons to an energy of $980 \mathrm{GeV}$, different aspects have to be considered. In this section we describe how the protons are extracted from hydrogen gas, and outline the more sophisticated production of antiprotons. The produced particles then have to be accelerated to an energy of $980 \mathrm{GeV}$, which requires a combination of different accelerator techniques each for a special energy range (see figure 2.2).

The protons are produced by the use of a $\mathrm{H}^{-}$ion source. The process starts at a magnetron, which creates the negative hydrogen ions by surface ioniziation effects. A magnetron consists of a cathode whose surface is coated with Cesium and an anode. The potential difference causes electrons at the surface of the metal cathode to overcome the work function of the metal, which is lowered by the Cesium film. A magnetic field perpendicular to the electrical field forces free electrons to travel in long spirals. Hydrogen gas is then injected into the volume between anode and cathode. The free electrons ionize the hydrogen gas so that the conditions inside the cavity create a dense plasma. Protons of the plasma are accelerated towards the cathode, collide with its surface, capture by different mechanisms two electrons, and are then extracted as $\mathrm{H}^{-}$ions from the magnetron. This ion beam leaving the magnetron continues its path by entering the Cockcraft-Walton accelerator column in which a series of electrostatic lenses accelerate the ion beam to an energy of $E=750 \mathrm{keV}$.

The $\mathrm{H}^{-}$ions are then passed to a Linear Accelerator (Linac) which is approximately $150 \mathrm{~m}$ long and consists of cascaded radio frequency cavity resonators that accelerate the ions. At the end of the Linac the ion beam is stable enough so that the electrons can be stripped off by passing through a carbon foil, leaving only a beam of protons. The next device in the sequence is the booster. The booster, a $75 \mathrm{~m}$ radius circle synchrotron, accelerates the protons in several circulations to an energy of $8 \mathrm{GeV}$ and sends them to the Main Injector. The Main Injector is a synchrotron seven times the circumference of the Booster and serves four functions. It accelerates the protons from $8 \mathrm{GeV}$ to $150 \mathrm{GeV}$, it provides $120 \mathrm{GeV}$ protons for antiproton production, it transfers and cools the antiprotons from the Antiproton Source, and it injects protons and antiprotons into the Tevatron. 


\section{FERMILAB'S ACCELERATOR CHAIN}

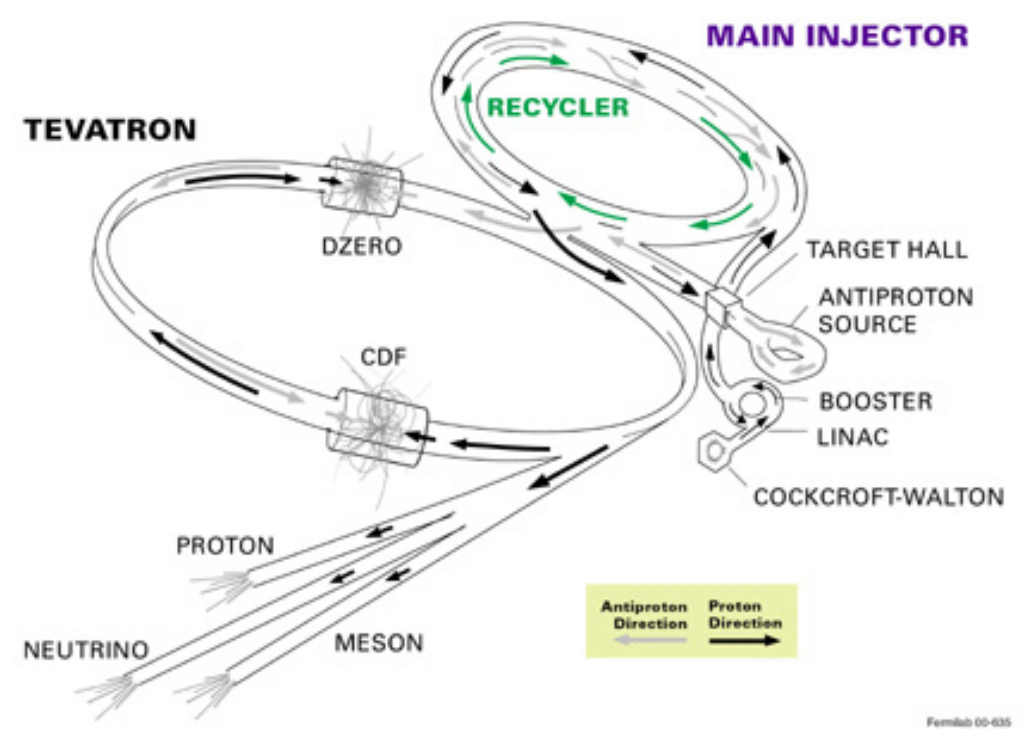

Figure 2.2: Schematic overview on the accelerator chain at the Fermilab.

To produce antiprotons, $120 \mathrm{GeV}$ proton beams from the Main Injector are transferred to the Antiproton Source and diverted into a nickel target. In these high-energetic collisions a wide range of secondary particles is produced including many antiprotons which are captured, focused and stored. After accumulating a sufficient number of antiprotons, typically $50 \cdot 10^{10}$, with a kinetic energy of about $8 \mathrm{GeV}$ they are sent to the recycler. To produce a tightly confined high energy beam only little spread in energy between the particles is allowed. Therefore, the process of electron cooling is used to shrink the size, divergence, and energy spread of the stored antiproton beam without removing particles from the beam. Since the number of particles remains unchanged and the space coordinates and their derivatives are reduced, this means that the phase space occupied by the stored particles is compressed. A stored antiproton beam is overlapped with a nearly monochromatic and parallel electron beam in one of the straight sections of the recycler. The velocity of the electrons is made equal to the average velocity of the antiprotons. The antiprotons undergo Coulomb scattering in the electron gas and lose energy, which is transferred from the antiprotons to the co-streaming electrons until some thermal equilibrium is attained.

When the antiprotons and the protons are accelerated by the Main Injector to the desired energy of $150 \mathrm{GeV}$ they are injected into the final acceleration chain, the Tevatron, which is a superconducting synchrotron with $1 \mathrm{~km}$ in radius. It accelerates the two oppositional circulating particle beams to a final energy of $980 \mathrm{GeV}$ by a series of radio frequency (RF) cavities. The orbits of the circulating protons and antiprotons are arranged such that the beams only cross at two points, where the large collider detectors D $\varnothing$ and CDF are placed.

Since the acceleration by RF cavity resonators requires single particle packages to 


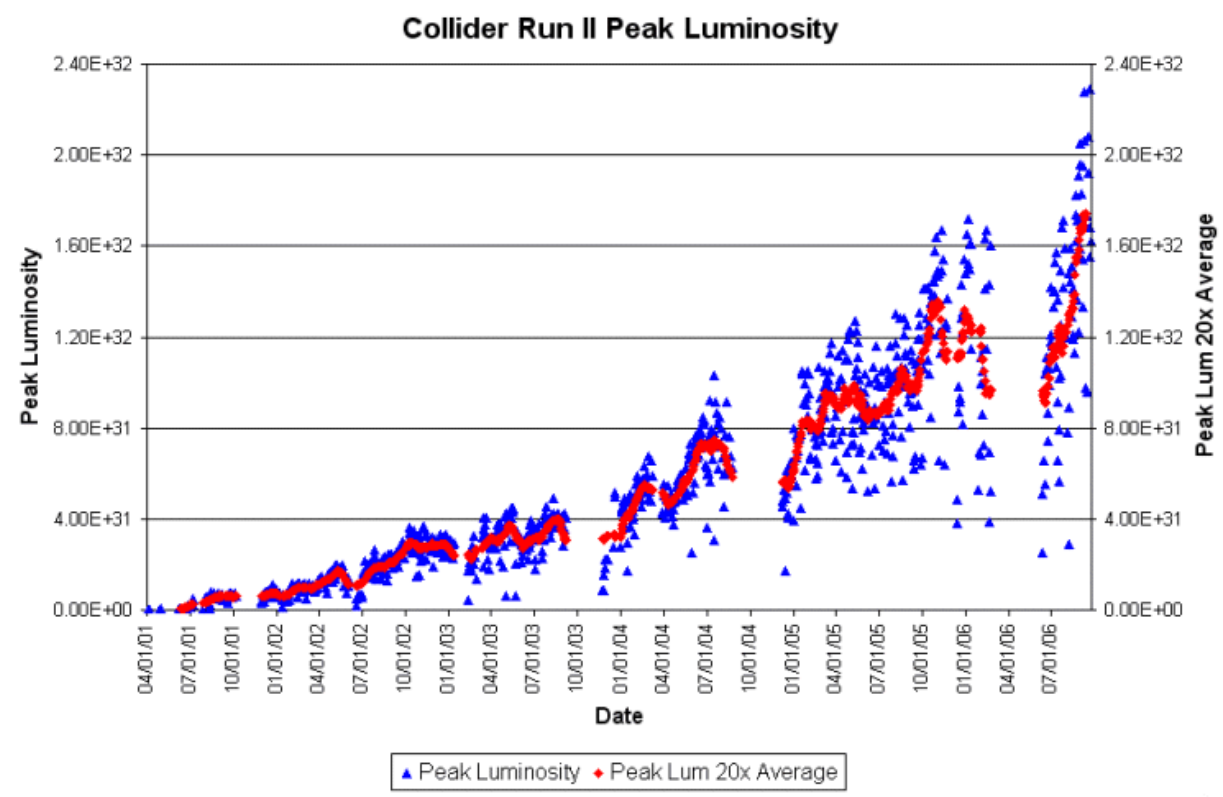

Figure 2.3: The collider run II peak luminosity as a function of the time. The blue squares show the peak luminosity at the beginning of each store. The red triangles display the average over 20 peak values.

come through with a certain distance in between to adjust the accelerating voltage phase, the protons and antiprotons are injected into the Tevatron in the form of bunches. Each of the 36 proton and 36 antiproton bunches in a temporal distance of 396 ns contains billions of particles and needs a certain time to circulate the Tevatron. At a final energy of $980 \mathrm{GeV}$ the revolution time of such a bunch is about $20 \mu \mathrm{s}$.

The ability of a collider to produce collisions is quantified by the instantaneous luminosity $\mathcal{L}$ :

$$
\mathcal{L}=n \cdot f \cdot \frac{N_{p} N_{\bar{p}}}{4 \pi \sigma_{x} \sigma_{y}}
$$

where $n$ is the number of bunches, $f$ is the revolution frequency for a single bunch traveling at the speed of light, $N_{p}$ and $N_{\bar{p}}$ are the number of protons and antiprotons per bunch, and $\sigma_{x}$ and $\sigma_{y}$ represent the average transverse width of the bunch. The instantaneous luminosity is usually measured in units of $\mathrm{cm}^{-2} \mathrm{~s}^{-1}$. The event rate $n$ for a certain physical process with a cross section $\sigma$ is given by the product $n=\mathcal{L} \cdot \sigma$. The total number of events $N$ is then obtained by using the time integrated luminosity $N=\sigma \int \mathcal{L} d t$. Tevatron Run II is planned to continue until September 2009 with projected ranges of an integrated luminosity from 4.4 to $8.5 \mathrm{fb}^{-1}[17]$.

Since the instantaneous luminosity exponentially decreases with time as the beam degrades due to collisions between protons and antiprotons and interactions with residual gas in the beam pipe, the luminosity does not remain constant. Figure 2.3 


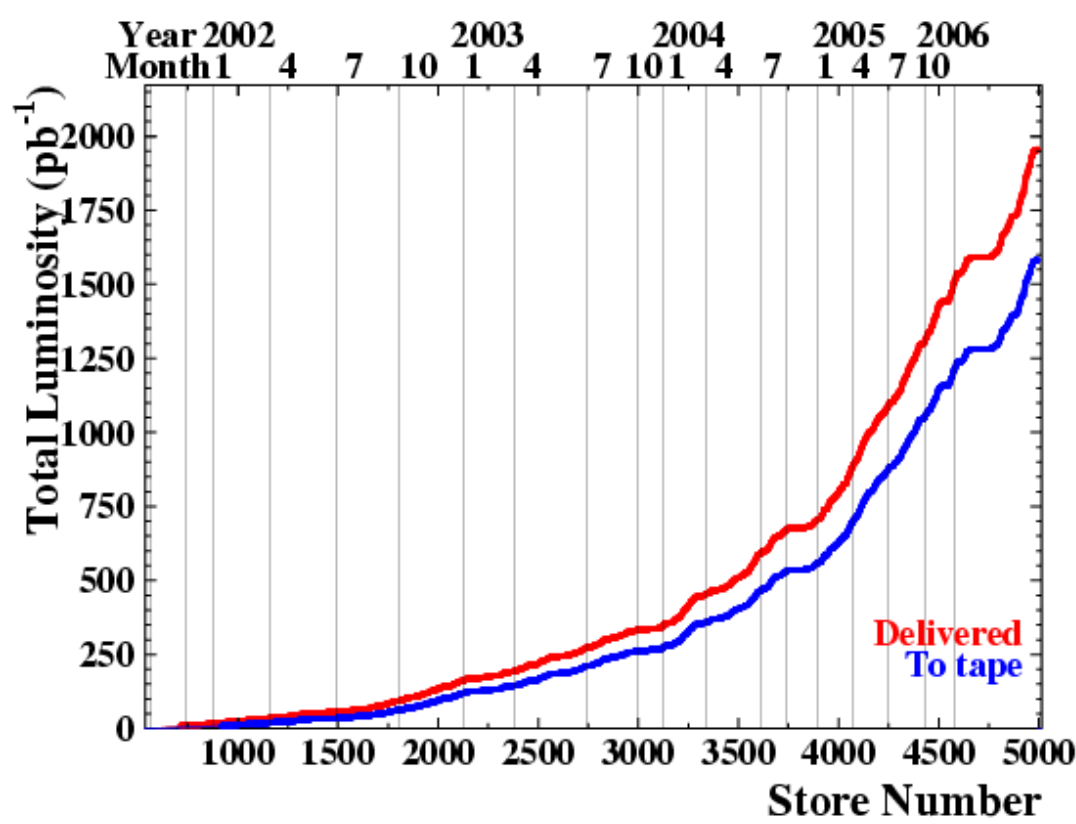

Figure 2.4: Evolution of the extracted data amount at CDF. The upper curve represents the delivered, the lower one the recorded integrated luminosity.

shows the peak luminosity at the beginning of each store, the time between two fillings, as a function of time. The average peak luminosity steadily increases due to an improved Tevatron performance. The target range of $\mathcal{L}=(1.6 \ldots 2.7) \cdot 10^{32} \mathrm{~cm}^{-2} \mathrm{~s}^{-1}$ [17] has been reached. The development of the delivered and recorded integrated luminosity, representing the amount of collected physical data, is shown in figure 2.4. The data used in this analysis are taken from March 2002 to February 2006, corresponding to an integrated luminosity of $955 \mathrm{pb}^{-1}$. This figure is lower than the recorded luminosity in that time period since good run criteria (3.1) are applied.

\subsection{The Collider Detector at Fermilab}

The collider detector at Fermilab (CDF) which is located at one of the two collision points of the proton and antiproton beams, is an azimuthally and forward-backward symmetric general purpose solenoid detector [20]. It combines precision charged particle tracking with fast projective calorimetry and fine grained muon detection to record as much information as possible about the results of proton-antiproton collisions.

Figure 2.5 shows a cutaway view of the CDF II detector with its different components. The tracking system is contained in a superconducting solenoid with a radius of $1.5 \mathrm{~m}$ and $4.8 \mathrm{~m}$ in length, generating a $1.4 \mathrm{~T}$ magnetic field parallel to the beam axis. The calorimetry and muon systems are all outside of the solenoid. The detector is described by right-handed spherical coordinates where the $z$-coordinate points along the proton direction. The polar angle $\theta$ is measured from the $\mathrm{z}$ direc- 


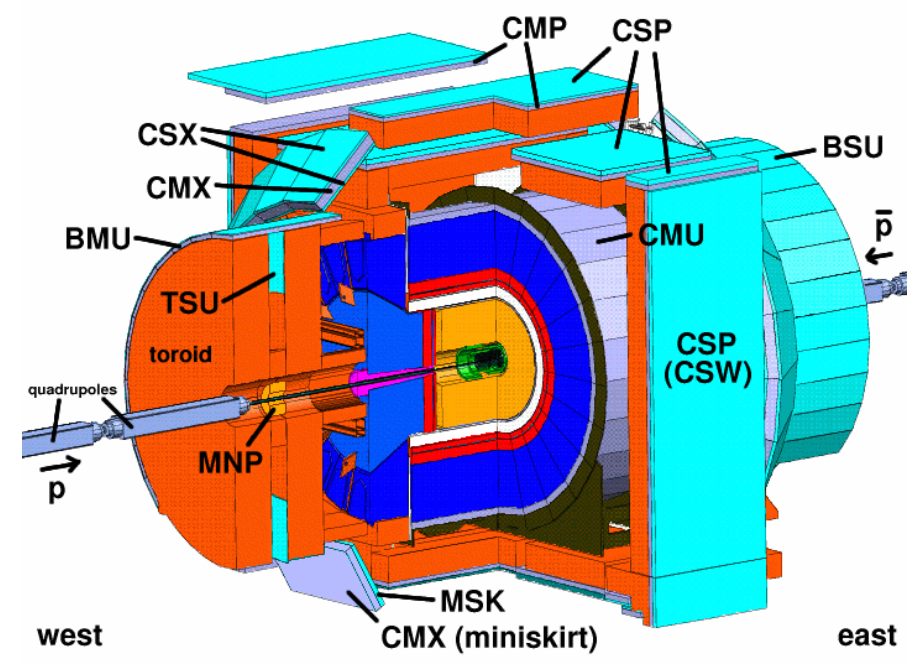

(a)

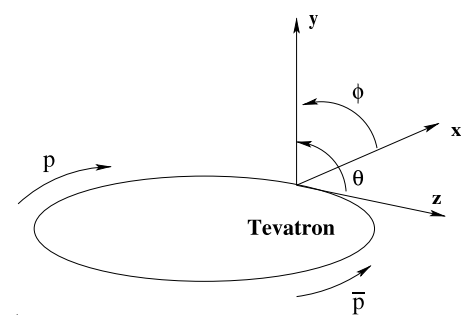

(b)

Figure 2.5: (a) Schematic view of the CDF II detector with its different components. The inner green and orange parts represent the tracking system and the blue ones the calorimeters. The labels refer to the different components of the muon system. (b) The CDF II coordinate system.

tion, the azimuthal angle $\phi$ is measured from the Tevatron plane, see figure 2.5. The pseudo-rapidity is then defined as:

$$
\eta=-\ln \left(\tan \frac{\theta}{2}\right) .
$$

The following sections describe the main components of the CDF II detector, the tracking system, the calorimetry and the muon system.

\subsubsection{Tracking System}

The tracking system of the CDF detector consists of four parts schematically presented in figure 2.6. The inner tracker consists of a silicon microstrip system arranged like concentric barrels in a row, and provides micro-vertex detection. It is surrounded by the Central Outer Tracker (COT), an open cell drift chamber.

The silicon layer closest to the beam pipe at a radius of $r=1.5 \mathrm{~cm}$ from the beam pipe center, Layer 00 [21], is a radiation hard, single sided silicon microstrip detector. The next five layers between radii of 2.4 and $10.7 \mathrm{~cm}$ constitute the Silicon Vertex Detector (SVX II) system [22], consisting of double sided silicon wafers. Three of the layers combine an $r$ - $\phi$ measurement on one side with a $90^{\circ}$ stereo measurement on the other, the remaining two layers combine $r$ - $\phi$ with small angle stereo at $1.2^{\circ}$. The twisted stereo measurements offer additional information in the $r-z$ plane allowing for a 3 dimensional tracking in the SVX II.

In the central region, $\eta \leq 1.0$, a single Intermediate Silicon Layer (ISL) [23] is placed at a radius of $22 \mathrm{~cm}$. In the plug region, $1.0 \leq|\eta| \leq 2.0$, two further ISLs 
CDF Tracking Volume

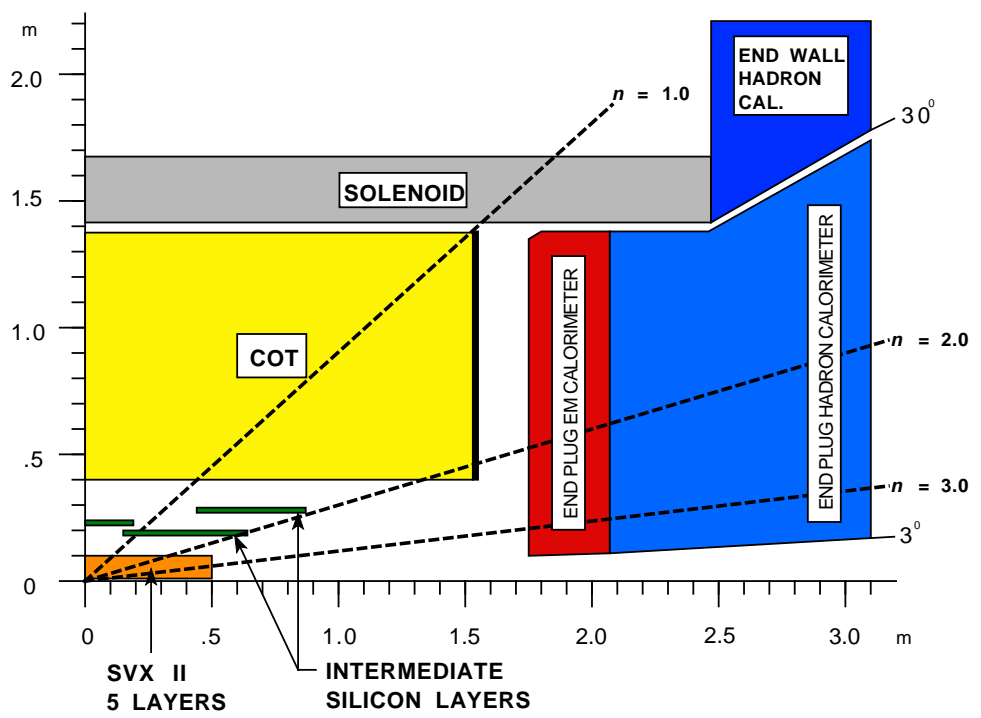

Figure 2.6: Longitudinal view of the CDF II tracking system and the plug calorimetry.

are placed at radii of $20 \mathrm{~cm}$ and $28 \mathrm{~cm}$. SVX II and ISLs, also consisting of double sided silicon detectors, provide stand-alone tracking over the full region $|\eta| \leq 2.0$.

The silicon microstrip system is surrounded by the COT [24], a $3.1 \mathrm{~m}$ long cylindrical drift chamber that covers the radial range from $44 \mathrm{~cm}$ to $132 \mathrm{~cm}$ and the $\eta$-range for $|\eta| \leq 1$. The COT is made up of four axial layers, where the wires in each cell are parallel to the $z$-axis providing $r$ - $\phi$ information, and four stereo layers, where the wires are $3^{\circ}$ offset from the $z$-axis offering $r-z$ information. The cells are filled with a 50-50 mixture of argon and ethan gas allowing to limit the drift time to less than $100 \mathrm{~ns}$, which is important to make a trigger decision as fast as possible.

\subsubsection{Calorimetry}

Outside the solenoid, scintillator-based calorimetry allows the measurement of the particle and jet energies by fully absorbing all particles except muons and neutrinos. The system consists of five calorimeter units: the Central ElectroMagnetic (CEM) [25] and Central HAdron (CHA) [26] calorimeters, covering the $\eta$-range up to 1.1, the endwall hadron calorimeter and the end-plug electromagnetic and hadron calorimeters [27], which complete a coverage of $2 \pi$ in azimuth corresponding to $-3.64 \leq \eta \leq 3.64$.

The CEM, which sits directly outside the solenoid, is a lead and scintillator sampling device with a unit layer composed of $4.5 \mathrm{~mm}$ lead and $4 \mathrm{~mm}$ scintillator. There are 23 layers in depth for a total thickness of about 21 radiation length at normal incidence. The detecting elements are arranged in a tower geometry pointing back towards the interaction region. The $\mathrm{CHA}$ is a 23 layer iron and scintillator sampling device with a unit layer composed of $5 \mathrm{~cm}$ iron and $6 \mathrm{~mm}$ scintillator. The construction of the two central calorimeters provides an energy resolution of 
$13.5 \% / \sqrt{E} \oplus 2 \%$ in the CEM $[25]$ and $75 \% / \sqrt{E} \oplus 3 \%[26]$ in the CHA calorimeter for single parton response.

\subsubsection{Muon System}

The muon system [28] is located outside the calorimetry to ensure that all particles except for muons and neutrinos are absorbed in the different calorimeters. Four systems of scintillators and proportional chambers are used for detection of muons over the region $|\eta| \leq 2.0$. The Central MUon Detection (CMU) system consists of four layers of planar drift chambers which cover $|\eta| \leq 0.6$ and is able to detect muons with a transverse momentum $p_{T} \geq 1.4 \mathrm{GeV} / c$. The Central Muon uPgrade (CMP), forming a box around the central detector, consists of a second set of muon chambers located outside the magnet return yoke, which constitutes another $0.6 \mathrm{~m}$ of absorbing steel. It detects tracks of muons with $p_{T} \geq 2.0 \mathrm{GeV} / c$ in the same $\eta$-range than the CMU. The Central Muon eXtension (CMX) consists of conical sections of drift tubes and scintillation counters located at each end of the central detector and extending in polar angle from $42^{\circ}$ to $55^{\circ}$. At $55^{\circ}$ the CMX system slightly overlaps the coverage provided by the central muon system and extends its pseudo-rapidity coverage from 0.65 to 1.0. The fourth muon system, the Intermediate MUon detection system (IMU), completes a muon identification up to $|\eta|=2.0$ by triggering on muons with $|\eta| \leq 1.5$ and identifying offline muons with $1.5 \leq|\eta| \leq 2.0$. Table 2.1 summarizes the main properties of the different muon chambers.

\begin{tabular}{|c|c|c|c|c|}
\hline & CMU & CMP & CMX & IMU \\
\hline$\eta$-range & $|\eta| \leq 0.6$ & $|\eta| \leq 0.6$ & $0.6 \leq|\eta| \leq 1.0$ & $1.0 \leq|\eta| \leq 1.5(2.0)$ \\
drift tube length & $226 \mathrm{~cm}$ & $640 \mathrm{~cm}$ & $180 \mathrm{~cm}$ & $363 \mathrm{~cm}$ \\
max. drift time & $800 \mathrm{~ns}$ & $1.4 \mu \mathrm{s}$ & $1.4 \mu \mathrm{s}$ & $800 \mathrm{~ns}$ \\
min. muon $p_{T}$ & $1.4 \mathrm{GeV} / c$ & $2.2 \mathrm{GeV} / c$ & $1.4 \mathrm{GeV} / c$ & $1.4-2.0 \mathrm{GeV} / c$ \\
\hline
\end{tabular}

Table 2.1: Design parameters of the CDF II muon detectors.

\subsection{The CDF Trigger System}

Although the design of the Tevatron offers the possibility to run with $108 \times 108$ bunches, corresponding to a clock cycle of $132 \mathrm{~ns}$ and a crossing rate of $7.6 \mathrm{MHz}$, the system currently operates with $36 \times 36$ bunches. The resulting crossing rate of $2.5 \mathrm{MHz}$ inside the CDF detector leads to a tremendous amount of information produced by the detector in each collision. Since the tape writing speed is limited to about $100 \mathrm{~Hz}$, a desicion system or trigger is required to extract events of most interest from the large number of minimum bias events. The CDF trigger is a three level system consisting of two hardware levels both of which are buffered (L1 and L2) and a software trigger running on a Linux PC farm. The functional block diagram in figure 2.7 gives an overview of the CDF II data flow. 
Dataflow of CDF "Deadtimeless"

Trigger and DAQ

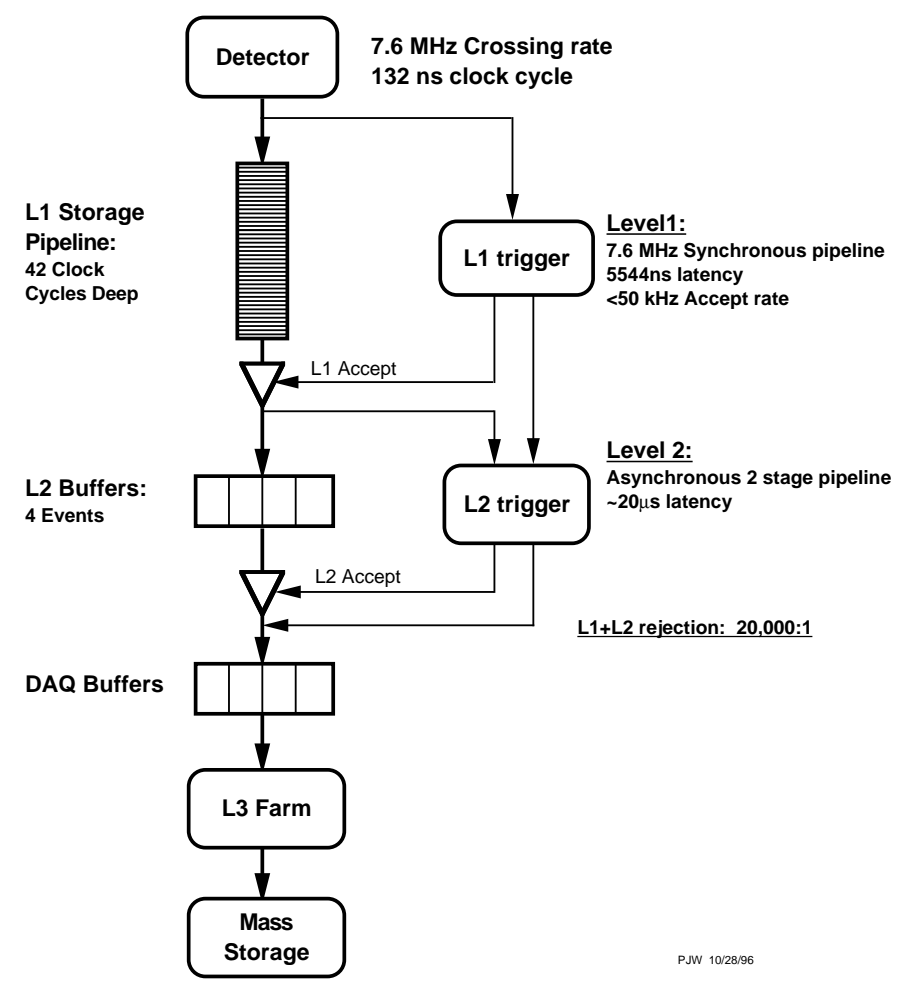

Figure 2.7: Functional block diagram of the CDF II data flow.

A crossing rate of $2.5 \mathrm{MHz}$ implies a bunch distance of $396 \mathrm{~ns}$ over the whole Tevatron ring, which would lead to a larger bunch number than 36. Actually, the bunches are arranged in three groups (trains), which circle the ring in a certain distance, abort gaps of $2.6 \mu \mathrm{s}$, from each other. During a train pass-by the crossing rate is $2.5 \mathrm{MHz}$ followed by the abort gap of $2.6 \mu$ s to the next train. Thus, the level 1 trigger (L1) has to deal with a decreased effective crossing rate of $1.7 \mathrm{MHz}$, but nevertheless is designed to handle rates up to $7.6 \mathrm{MHz}$ as can be seen from figure 2.7. The first trigger level is based on preliminary detector information. Three synchronous systems run parallel to examine an event with respect to fast reconstructed tracks in the COT, in the muon chambers, and a first estimate of the amount of energy deposited in the calorimeters. A desicion is made by simply counting these objects for every beam crossing and permits to limit the rate for accepted events to $18 \mathrm{kHz}$. The second trigger level (L2) provides a limited event reconstruction based on more specific event information and additional tracking information from the silicon detector SVX II as well as from the shower maximum strip chamber in the central calorimeter. The trigger rate can therewith be further reduced to about $300-500 \mathrm{~Hz}$. The last step to the level three (L3) software trigger provides a full read out of the event. The event data is passed to a PC farm where a complete version of the offline reconstruction is performed. The L3 trigger decision 
is then made based on the particle content and event topology. The output rate of the level three trigger of about $100 \mathrm{~Hz}$ now allows to write the remaining events to permanent storage. 


\section{Chapter 3}

\section{Event Selection and Reconstruction}

In this chapter we describe the complex process of extracting the physics we are interested in from the wide range of electronical detector responses. The finally obtained data samples should contain as much signal events as possible and the measured final state objects should correspond best to the primarily produced physical particles. Therefore, several event selection cuts are applied to the samples that passed the different trigger levels (see section 2.3), and the observed objects are corrected for detector and physics effects. Based on these detected final state objects, we need to reconstruct the kinematics of the produced top pairs since we intend to analyze their rapidities. The sections below discuss the most important jet corrections and selection cuts, followed by a description of the full reconstruction of top pairs.

\subsection{Event Selection in the Lepton + Jets Channel}

In this thesis only top pairs decaying in the "lepton+jets" channel are considered, where one top quark decays hadronically $\left(t \rightarrow b W \rightarrow b q_{1} \bar{q}_{2}\right)$ into a $b$ quark and two quarks $(q=u, d, s, c)$, and the second one decays semileptonically $(t \rightarrow b W \rightarrow b \ell \nu)$ into a $b$ quark, a charged lepton $(e, \mu)$, and the corresponding neutrino. The choice of this channel constitutes a compromise with respect to backgrounds, branching ratio, jet multiplicity, and missing transverse energy. Compared to the dilepton channel (both top quarks decay semileptonically), the backgrounds in the lepton+jets channel are considerably higher, but still manageable. On the other hand, two neutrinos contribute to the missing transverse energy measured in the dilepton channel, complicating the reconstruction. The lepton + jets channel offers a much larger branching ratio of about $30 \%$. As we will discuss in section 3.2 a jet multiplicity of at least four in the lepton+jets channel complicates the reconstruction of the top quarks compared to the dilepton channel, but in the all-hadronic case it would be even worse.

The Feynman diagram of a top pair produced via quark-antiquark annihilation for a decay in the lepton+jets channel is shown in figure 3.1. The partons in the final state, charged lepton, neutrino, and four quarks, can be associated with certain 
detector responses. The event signature in the detector is characterized by one isolated charged lepton $(e, \mu)$, at least four jets $\left(b, \bar{b}, q_{1}, \bar{q}_{2}\right)$, and missing transverse energy due to the undetectable neutrino $(\nu)$.

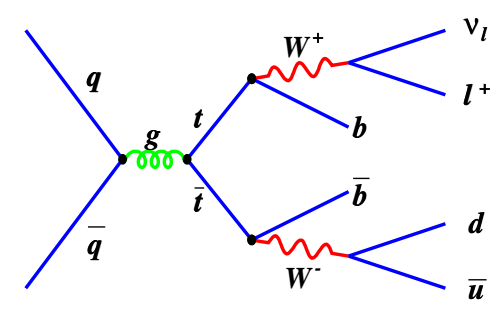

Figure 3.1: Feynman diagram of top-pair production via quark-antiquark annihilation, where the top pair decays in the lepton+jets channel.

Therefore, we use the data stream including all inclusive high- $p_{T}$ lepton samples (stream B). The analyzed data for this thesis were taken from March 2002 to February 2006. In the first period until August 2004 the data were reprocessed with CDFSOFT2 [33] version 5.3.3 and stripped into two data sets containing CEM electrons (bhel0d) or CMUP and CMX muons (bhmu0d). During the second period, till February 2006, data were reprocessed with CDFSOFT2 version 6.1.2 and stored in bhel0h/bhel0i or bhmu0h/bhmu0i, respectively. Electron data (bhelXX) have to pass the high- $p_{T}$ central electron Level3 trigger ELECTRON_CENTRAL_18_V, while the muon data (bhmuXX) have to pass one of the high- $p_{T}$ central muon Level3 trigger MUON_CMUP18 or MUON_CMX18. The main requirements of these triggers are tracks in the COT and a matching signal in the corresponding detector component: in the CEM for electrons, in the CMU and CMP for CMUP muons, and in the CMX for CMX muons.

During data taking each detector component has to work properly to verify high quality of data. Since the detector system is quite complex, the process of data taking is not continuous but often interrupted by an insufficiently operating subsystem. On the time scale this means, that one Tevatron store (see chapter 2.1) is partitioned into several runs, periods of efficient data taking. The runs are monitored both online and offline for all subsystems separately, thus only runs in which certain detector components were active and determined to be good enter the goodrun list [32]. Each physics analysis demands different active detector components so there are several goodrun lists to maximize the usable data amount for everyone. For our $t \bar{t}$ analysis in the lepton + jets channel we require the following detector components to be active: the silicon detector, the COT, the muon system, and the calorimeter system. Therefore, we use the goodrun list version 13 [34] of the CDF top quark group. The total data set used in this analysis corresponds to an integrated luminosity of $\mathcal{L}=955 \pm 57 \mathrm{pb}^{-1}$ for $\mathrm{CEM} / \mathrm{CMUP}$ and $\mathcal{L}=941 \pm 56 \mathrm{pb}^{-1}$ for CMX.

Although the main characteristics, like they are observed by the detector, of events that pass the triggers are similar, data samples still contain a wide range of different event topologies including signal as well as background events. Thus, data 
have to pass a set of several selection cuts in order to enrich the signal fraction and to reduce the background fraction and some objects are corrected for detector and physics effects. In the following the main elements of the event selection as well as jet corrections are outlined, following the suggestions of the lepton + jets group for the CDF software version 5.3.3 [35].

\section{Lepton Identification:}

The signature of $t \bar{t}$ events in the lepton + jets channel is characterized by one highenergy charged lepton. The charged leptons are classified according to the detector component they are observed in. The required lepton may be a tight electron detected in the CEM calorimeter or a tight muon in the CMU as well as in the CMP, or in the CMX. Electrons are accepted with a transverse energy $E_{T} \geq 20 \mathrm{GeV}$. In the case of muons, where energy is not measurable due to a minimal energy loss in the calorimeters, we only require tracks in the COT and muon chambers, whose curvature yields a transverse momentum $p_{T} \geq 20 \mathrm{GeV} / c$. The isolation is a further crucial parameter to ensure that not a part of a jet was misidentified as a charged lepton. It is defined as the fraction of non-leptonic transverse energy in a cone with radius $\Delta R=0.4$ in the $\eta-\phi$-plane around the charged lepton compared to the electron $E_{T}$ or muon $p_{T}$, respectively. The isolation of tight leptons has to be less or equal to 0.1. Tight leptons are characterized by further identification requirements that have to be fulfilled by several observables. They are described in more detail in reference [36,37].

To verify the charged leptons resulting from the hard process, events are rejected if the electron was generated by the conversion of a photon into an $e^{+} e^{-}$pair or if the muon was observed to be a cosmic one.

\section{Dilepton Veto:}

Since our event signature requires only one tight lepton, events which have one primary (tight) and one secondary (tight or loose) charged lepton are vetoed. The class of loose leptons is defined by the dilepton group, see [38].

\section{$z$ Vertex Cut:}

To ensure that the high-energy lepton comes from the event $z$ vertex, we reject events for which the charged lepton $z_{0}$ is greater than $5.0 \mathrm{~cm}$ away from the $z$ vertex. The event $z$ vertex is the vertex on the $z$ axis closest to maximum- $E_{T}$ tight lepton with at least two good COT tracks. In the case that there is no such vertex found, $z$ is set to the high-energy lepton $z_{0}$. By definition, this requirement is always satisfied.

\section{$Z$ Boson Veto:}

$Z$ boson events are suppressed by removing all events, where a tight lepton and a second object form an invariant mass $M$ within a window of the $Z$ mass. The default window is $(76 \leq M \leq 106) \mathrm{GeV} / c^{2}$. If the tight lepton is an electron, the second object may be an isolated electromagnetic object, a reclustered jet with electromagnetic fraction greater than 0.95, or an opposite-signed isolated track. If the tight lepton is a muon, the second object may be an isolated muon or an opposite-signed isolated track. 


\section{Jets:}

The quarks produced at parton level or radiated hard gluons hadronize to colorless objects due to the confinement. These hadrons again decay into several secondary particles, which are absorbed in the hadron calorimeter. Thus, the detector merely observes amounts of energy depositions in the different calorimeter towers. The JETCLU algorithm [39] identifies these energy depositions as separate objects, jets, being considered to contain information about the primary parton that initialized the observed particle shower. This is done by clustering energy depositions in the calorimeters within a certain cone of radius $\Delta R=0.4$ in the $\eta-\phi$ plane with respect to the event $z$ vertex. To receive a better description of the primary parton jet from the clustered detector object, several steps of jet corrections have to be applied, which correct for detector effects as well as physics effects. The corrections are classified into different levels, whereby a higher level includes the lower ones. Level 4 contains an $\eta$ dependent correction to flatten the calorimeter response which is not uniform in pseudo-rapidity due to the spatial separation of calorimeter components. It also considers corrections for multiple $p \bar{p}$ interactions: with a luminosity of $10^{32} \mathrm{~cm}^{-2} \mathrm{~s}^{-1}$ we expect on the average three to four interactions per beam crossing. Thus, a fraction of events can have at least one overlapping event. Its energy may fall into the jet clustering cone of the hard interaction and must be subtracted. Since we intend to reconstruct the produced $t \bar{t}$-pair for our analysis, the jets need to be further corrected, up to level 7. This includes corrections for nonlinearity and energy-loss in the uninstrumented regions of the central calorimeter (absolute jet energy scale, level 5), and underlying events (level 6) are taken into account by subtracting energy from spectator interactions that fall inside the jet clustering cone. The out-of-cone correction at level 7 eventually considers initiating partons generated during the fragmentation, that may fall outside the cone chosen for the clustering algorithm. This energy must be added to the particle jet to get the parton jet energy. A more detailed description of all jet corrections can be found in [40].

For our analysis we require an event signature containing at least 4 tight jets, which are defined after the level 4 corrections by a minimal transverse energy $E_{T} \geq$ $15 \mathrm{GeV}$ and an absolute value of pseudo-rapidity $|\eta|<2.0$.

\section{Missing Transverse Energy:}

Neutrinos produced in an event are not detectable due to a very small cross section for an interaction with detector material, so the appearance of a neutrino manifests as missing transverse energy in the event. The missing $E_{T}\left(E_{T}\right)$ is defined by

$$
\vec{\not}_{T}=-\sum_{i} E_{T}^{i} \hat{n}_{i}, i=\text { calorimeter tower number with }|\eta|<3.6
$$

where $\hat{n}_{i}$ is a unit vector perpendicular to the beam axis and pointing at the $\mathrm{i}^{\text {th }}$ calorimeter tower. The absolute value of this vector is defined as $E_{T}=\left|\vec{H}_{T}\right|$. Since muons deposit only a small fraction of their energy in the calorimeter, thus missing $E_{T}$ has to be corrected in case of muon events. The transverse muon momentum 
has to be considered in the sum on the right hand side of equation 3.1, and, at the same time, the energy deposits by the muon in the calorimeter towers have to be removed. $\mathbb{E}_{T}$ is corrected for the jet energy corrections for all jets with $|\eta|<2.4$ and level 4 corrected jet energy $E_{T} \geq 8 \mathrm{GeV}$. To be accepted, an event has to yield a corrected missing transverse energy of at least $20 \mathrm{GeV}$. For the full reconstruction of the $t \bar{t}$ pair the missing transverse energy is only corrected up to level 6 , because level 7 corrections lead to double-counting of the out-of-cone energy.

\section{$b$ Tagging:}

Since the decay signature of the $t \bar{t}$ pair contains two $b$ quarks, we expect to observe at least one jet that stems from a $b$ quark. These $b$ jets are tagged by the SecVtx [41] algorithm using the fact, that $b$ quarks hadronize into $b$ hadrons having relatively long lifetimes, on average $1.5 \mathrm{ps}$, and therefore travel an observable distance before decaying. The average distance of these displaced secondary decay vertices from the primary interaction point is about $7.5 \mathrm{~mm}$ for $b$ hadrons produced in top decays at the Tevatron. The SecVtx algorithm looks for tracks in the cone of each jet with corrected $E_{T} \geq 15 \mathrm{GeV}$ and detector $|\eta|<2.0$, that do not match to the primary interaction vertex, but intersect in such a secondary, displaced vertex. Figure 3.2 gives a schematic overview of event tracks containing a secondary vertex and illustrates the definition of the main parameters, the impact parameter $d_{0}$ and the distance between the two vertices in the $\eta-\phi$ plane, $L_{x y}$.

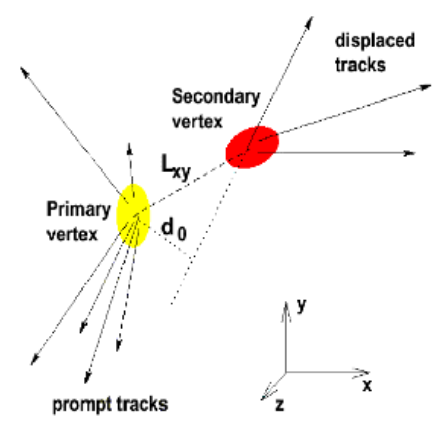

Figure 3.2: Schematic view of an event containing a secondary vertex. The two main parameters used by the SecVtx algorithm are illustrated, the 2-D decay length $L_{x y}$ and the impact parameter $d_{0}$.

Only tracks with a minimum number of hits in the silicon detectors and an impact parameter $d_{0}$ smaller than $0.3 \mathrm{~cm}$ are considered "good" and therefore taken into account. Since at least two tracks are needed to reconstruct an intersection, only events containing jets with at least two "good" tracks are considered "taggable". Once a secondary vertex is found, the distance $L_{x y}$ is calculated. To get tagged as a $b$ jet, the significance of the displacement $L_{x y} / \sigma_{x y}$ has to be equal or larger than 7.5. The event is accepted if at least one jet in the event is tagged as a $b$ jet. 
Chapter 3. Event Selection and Reconstruction

\begin{tabular}{|c|c|c|c|c|}
\hline & CEM & CMUP & CMX & $\Sigma$ \\
\hline bhel0d & 48 & - & - & 48 \\
bhel0h & 51 & - & - & 51 \\
bhel0i & 42 & - & - & 42 \\
\hline bhmu0d & - & 26 & 10 & 36 \\
bhmu0h & - & 21 & 11 & 32 \\
bhmu0i & - & 15 & 8 & 23 \\
\hline$\Sigma_{\text {tot }}$ & 141 & 62 & 29 & $\mathbf{2 3 2}$ \\
\hline
\end{tabular}

Table 3.1: Number of $t \bar{t}$ event candidates after performing the event selection. The values are shown for the six data samples corresponding to an integrated luminosity of $955 \mathrm{pb}^{-1}$, and with respect to the charged lepton type.

\section{Summary:}

Summarizing the main selection criteria, we require exactly one tight lepton $(e, \mu)$ with a transverse energy $E_{T} \geq 20 \mathrm{GeV}$ for electron candidates and with a transverse momentum $p_{T} \geq 20 \mathrm{GeV} / c$ for muon candidates. The events have to feature at least four tight jets which are characterized after the corrections (L4) by a minimal transverse energy $E_{T} \geq 15 \mathrm{GeV}$ and an absolute value of pseudo-rapidity $|\eta|<2.0$. At least one of them must be tagged as $b$ jet and the level 4 corrected missing transverse energy $Z_{T}$ of the event has to be at least $20 \mathrm{GeV}$. After performing the full event selection on the data set corresponding to an integrated luminosity of $955 \mathrm{pb}^{-1}, 232 t \bar{t}$ event candidates remain. Table 3.1 gives an overview of the number of these events in the different data samples with respect to the charged lepton type.

\subsection{Full Reconstruction of the Top Pair}

Analyzing the rapidity difference of the top pair requires the reconstruction of the complete four-vectors of both top quarks. Since several ambiguities occur during the reconstruction process, we have to find quality criteria describing which of the different possible event interpretations corresponds best to the true event shape. The main aspects of the used reconstruction method [42] are outlined in the following.

The reconstruction starts with the leptonically decaying $W$ boson $\left(W_{l e p} \rightarrow \ell \nu_{\ell}\right)$. The four-vector of the $W_{l e p}$ boson is the sum of the four-vectors of its decay products, the charged lepton and the neutrino. The four-vector of the charged lepton can be reconstructed almost perfectly, whereas for the neutrino, which is interpreted as the missing transverse energy in the event, only the transverse components are measured as $p_{T, \nu}=\vec{H}_{T}$. The missing $z$ component information in case of the neutrino can be determined from the $W$ mass constraint. Conservation of the invariant mass in the decay of the $W$ boson into a charged lepton and a neutrino yields a quadratic equation for the $z$ component of the neutrino momentum:

$$
P_{z, \nu}^{2}-2 \cdot \frac{\mu \cdot P_{z, \ell}}{E_{\ell}^{2}-P_{z, \ell}^{2}} \cdot P_{z, \nu}+\frac{E_{\ell}^{2} \cdot P_{T, \nu}^{2}-\mu^{2}}{E_{\ell}^{2}-P_{z, \ell}^{2}}=0 .
$$


Here $P_{z, \ell}$ and $E_{\ell}$ denote the $z$ component of the charged lepton $(e, \mu)$ momentum and its energy respectively. $\mu$ is defined via $m_{W}^{2} / 2+\cos (\Delta \phi) \cdot P_{T, \ell} \cdot P_{T, \nu}$, in which $\Delta \phi$ is the azimuthal angle difference between the momentum of the charged lepton and $\vec{H}_{T}$. The quadratic equation leads in general to two solutions $\left(N_{\text {sol }}=2\right)$ both of which have to be considered. If the reconstructed transverse $W$ boson mass $m_{T, W}$ is larger than the mass demanded by the $W$ mass constraint, the solution becomes complex and only the real part is taken as physical value of $\left|P_{z, \nu}\right|$, thus the number of solutions is one. To reconstruct now the semihadronically decaying top quark we need to add the four-vector of a jet representative of the $b$ quark to the already obtained four-vector of the $W_{l e p}$ boson. Since we observe at least four jets, there are at least 4 different possibilities to combine the $W$ boson with one of the jets. Taking into account the number of $P_{z, \nu}$ solutions, the total number of different hypotheses so far is given by $N_{\text {hyp }}=N_{\text {sol }} \cdot N_{\text {jets }}$.

For the hadronically decaying top quark the combinatorics of the remaining $\left(N_{\text {jets }}-1\right)$ jets determine the total number of hypotheses. Considering the hadronically decaying $W$ boson, we have to sum up two further jets to obtain the $W_{\text {had }}$ boson four vector. The choice of the first jet offers $\left(N_{\text {jets }}-1\right)$ possibilities, for the second one $\left(N_{\text {jets }}-2\right)$ alternatives remain. The fact, that permutations of the two chosen jets lead to identical $W$ boson four-vectors, yields a factor $1 / 2$ in the total number of hypotheses. To obtain the four-vector of the second top quark one last jet has to be added to each of the previously reconstructed four-vectors of the $W_{\text {had }}$ boson. For this still $\left(N_{\text {jets }}-3\right)$ possibilities remain. Finally, for a $t \bar{t}$ event with $N_{\text {jets }}$ observed jets, we obtain

$$
N_{\text {hyp }}=N_{\text {sol }} \cdot N_{\text {jets }} \cdot\left(N_{\text {jets }}-1\right) \cdot\left(N_{\text {jets }}-2\right) \cdot \frac{1}{2} \cdot\left(N_{\text {jets }}-3\right)
$$

different hypotheses, i.e. $N_{\text {hyp }}$ different alternatives to reconstruct the four-vectors of the two produced top quarks.

The challenge is now to find that hypothesis, that corresponds best to the kinematics of the produced $t \bar{t}$ pair. A sophisticated event simulation based on Monte Carlo methods (see chapter [?]) is used to derive a method that estimates, how well each hypothesis corresponds to the produced $t \bar{t}$ pair. The quality of a hypothesis is described by the quantity $\Psi$ which is defined as:

$$
\Psi=P_{\nu} \cdot \chi^{2} \cdot P_{b-l i g h t}
$$

This quantity combines different aspects of the reconstruction. The weighting factor $P_{\nu}$ takes into account that in $71 \%$ of all events with two real $P_{z, \nu}$ solutions, the one with the smaller value leads to the correct $W$ boson four vector. Thus, it is set to 0.29 for the hypothesis using the solution with the smaller value, and to 0.71 for the one with the larger solution for $\left|P_{z, \nu}\right|$. If we obtain only one $\left|P_{z, \nu}\right|$ solution, this value is arbitrarily set to one, since the value is equal for all the twelve hypotheses. The $\chi^{2}$ function considers the mass of the hadronically decaying $W$ boson, the two top quark masses $m_{t \rightarrow b \ell \nu}$ and $m_{t \rightarrow b j j}$, and the sum of the transverse 
energies of the reconstructed top quarks divided by the observed total transverse energy of the event. The quantity $P_{b-l i g h t}$ describes the light quark likeness of the jets assigned as $b$ jets and is given by:

$$
P_{b-l i g h t}=\left(\mathcal{P}_{t \rightarrow b \ell \nu}+\left(1-R_{t \rightarrow b \ell \nu}^{\prime}\right)\right) \cdot\left(\mathcal{P}_{t \rightarrow b j j}+\left(1-R_{t \rightarrow b j j}^{\prime}\right)\right)
$$

This light quark likeness considers two different aspects. $\mathcal{P}_{t \rightarrow b \ell \nu}$ and $\mathcal{P}_{t \rightarrow b j j}$ are the probabilities of the jets chosen to be the $b$ jets from the semileptonically and hadronically decaying top quarks to belong to the primary vertex, and thus to be rather a light quark jet. This probability is calculated with the JetProb package [43] and is based on the positive impact parameter of the tracks assigned to the jet in the $r-\phi$-plane. To decide, how much a hypothesis should be preferred in which the jets assigned to a $b$ jet have a SecVtx tag, the quality of the $b$ tag is described by the quantity $R^{\prime}$ [44] using a neural network (NN) b-tagger [45].

The parameter $\Psi$ is constructed in a way that the hypothesis which corresponds best to the produced top pair is the one with the smallest value for $\Psi$. In our analysis this hypothesis is chosen to be the selected event interpretation. A detailed discussion of the contributing parameters and studies estimating the quality of the reconstruction method can be found in reference [44]. 


\section{Chapter 4}

\section{Study of Top-Pair Production}

As regards the experiment, we are able to interpret several detector signals as produced particles by reconstructing tracks of charged particles and analyzing energy deposits in the calorimeters. We perform an event selection to reduce the fraction of non-t $\bar{t}$ events in the data samples, and a full reconstruction method extracts information about the kinematics of the produced top quark pair. To study the influence of these processes on the measured observable we need to simulate the data. This is done by using Monte Carlo methods to generate events of a certain physical process. These generated Monte Carlo event samples can then be subjected to a detector simulation and the full data processing. Thus, we obtain event samples that are in the same format as measured data. They are used to develop unfolding methods correcting for detector efficiency and reconstruction effects, since unfolded measurement results are directly comparable to theoretical predictions. In this chapter, we first give an introduction to the used Monte Carlo event generators, followed by the presentation of some properties of top-pair production at generator level.

\subsection{Monte Carlo Event Generators}

Monte Carlo event generators accumulate our understanding of the standard model of elementary particle physics into one package. Numerical simulations based on Monte Carlo methods are used to randomly generate hard parton interactions according to the probability density of phase space and the matrix element of certain physical processes. As can be seen in figure 4.1, events occurring in protonantiproton collisions at the Tevatron are not described only by the hard scattering process. Several stages have to be implemented in the event simulation to obtain results that are comparable to data. This is not done in one step, but rather by factorizing the full problem into a number of components, each of which can be handled reasonably accurately.

For a full simulation of top-pair events as they are analyzed in this thesis the following subprocesses are required to be included in the used event generator. The incoming beam partons, proton and antiproton, are characterized by a set of parton 


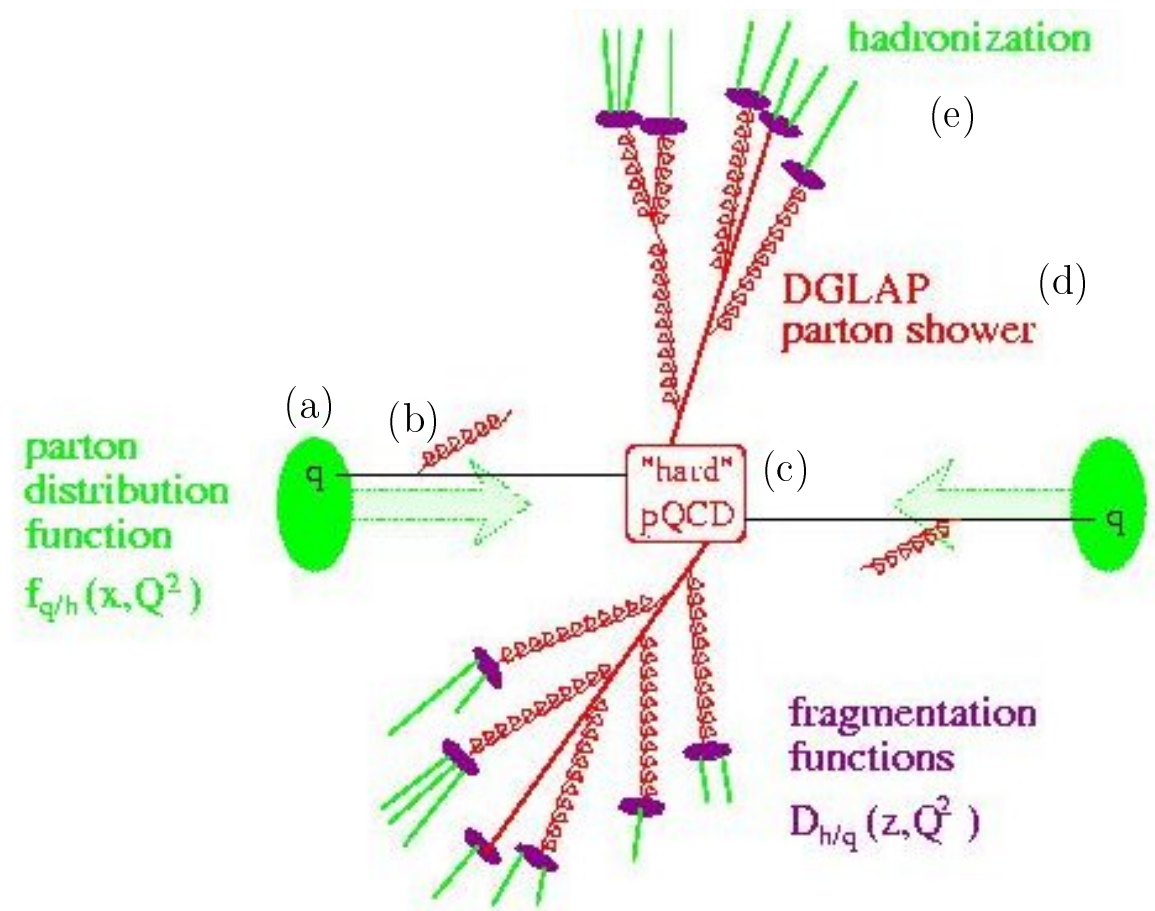

Figure 4.1: Schematic view of a QCD event showing the evolution of the produced particles. The incoming quark and antiquark are characterized by the parton distribution functions (PDF) of the beam particles $(p, \bar{p})$. The hard scattering process, taking place at high energies, i.e. at short distances, is exactly calculable by using perturbative methods. The parton showering is described by the DGLAP evolution equations. Since for increasing distances the strong coupling constant $\alpha_{s}$ becomes larger than 1, perturbation theory is no longer applicable. Thus hadronization has to be described by phenomenological models.

distribution functions $f\left(x, Q^{2}\right)$ (fig. 4.1 (a)) which define the partonic substructure in terms of flavor composition and momentum sharing. One shower initiator parton from each beam may start off a sequence of branchings, such as $q \rightarrow q g$ (fig. 4.1 (b)) building up an initial shower. One parton from each of the two initial showers enters the hard QCD process (fig. 4.1 (c)). Since this scattering process takes place at large momentum transfer scales $Q^{2}$ for which the strong coupling constant $\alpha_{s}$ is small, the cross section of the hard process can be computed exactly at least to lowest order perturbation theory. NLO calculations of the cross sections are in principle feasible, but lead to further problems concerning the parton showering in the process (see below). At the hard process a number of primary outgoing fundamental objects, e.g. a $t \bar{t}$ pair, is produced. The nature of this process, i.e. the color flow and the momentum transfer scale, determines the main characteristics of the whole event. The hard QCD process box in figure 4.1 also contains the calculable decay of the produced top quarks. Final state gluons (fig. 4.1 (d)) may be radiated off the top and $b$ quarks, they again branch into further colored partons $(q, g)$ to build up final state showers. These gluon radiations, as well as the initial showers, are mathematically described by the DGLAP QCD evolution equations [46], where the probability of radiating a gluon $(g \rightarrow g g / q \bar{q}, q \rightarrow q g / g q)$ is considered according to the Altarelli-Parisi splitting functions. The DGLAP equations yield, that parton cascades follow a strong ordering in transverse momentum, whereby initial state gluon radiation is evolved 
backward from the hard process to the proton. This behavior is implemented in the generators by using a certain angular ordering parameter. Since the branching of quarks and gluons are due to soft and collinear gluon emissions, the mathematical description of parton showers contains singularities. Thus, parton showering generators have to model these effects by using a generator dependent cut-off scale for the according ordering parameter. The parton showering regime is determined by the choice of this cut-off, which is chosen to stay away from non-perturbative physics, but still allows a sufficient showering to describe the process as realistic as possible, which even includes a simulation of interference effects. As a result of perturbative shower evolution, the partons are grouped in color singlet clusters that end up close in phase space. These preconfined clusters serve as starting point for the hadronization of the shower partons into color neutral hadrons. The hadronization process (fig. 4.1 (e)) takes place at a low momentum transfer scale $Q^{2}$ for which the strong coupling is larger than 1 and perturbation theory is not applicable. Thus, the transformation of partons into hadrons is difficult to describe due to the absence of a firm theoretical understanding. The generators try to implement the hadronization process by using different phenomenological models.

Depending on the included processes, we distinguish between parton shower (PS) generators, providing a full event simulations as described above, and matrix-element generators calculating only the cross section of the hard scattering process. In the following the different Monte Carlo generators used for this analysis are shortly described.

\section{Pythia:}

Pyтhia [47] is a program for the generation of high-energy physics events including PS, with emphasis on those where strong interactions play a role and therefore multihadronic final states are produced. The angular ordering demanded by the color coherence of the radiated virtual gluons during the parton showering is organized by using $Q^{2}=m^{2}$ as ordering variable. The hadronization process is described by the Lund string model. A string represents the color flux tube, stretched between a $q$ and a $\bar{q}$ due to the confinement of color charged particles. The string energy can be converted to further $q \bar{q}$ pairs if the invariant mass of the string is large enough. The chance for producing different flavors is dependent on the flavor mass, charmed and heavier quarks are hence not expected to be produced in the soft fragmentation. As quarks are quantum mechanically created in one point (local flavor conservation), the tunneling probability of the $q \bar{q}$ pair to get out to classically allowed regions as well as the chance that a given $q \bar{q}$ pair combination forms a specific meson has to be considered. The hadron production is treated as an iterative process which continues until the string is consumed. Additional gluon radiation during the fragmentation into hadrons is described as a kink on the string. If such a branching occurs the string is stretched between $q g$ and $g \bar{q}$ respectively. The hadrons produced in this way move basically in the direction of the kink, which is known as the string effect. 


\section{Herwig:}

HERWIG [48] is also a general purpose event generator for high-energy processes with particular emphasis on the detailed simulation of hadron emission reactions with interfering gluons. The used parton shower approach for initial and final state gluon radiation includes color coherence effects in all hard subprocesses and azimuthal correlations within and between jets due to gluon interference and polarization. As ordering parameter the branching angle between the branching shower parton and the radiated one is used. The hadronization is described by the cluster model following up the preconfined clusters resulting from the PS period. The clusters are differentiated by their mass, which has to be within a defined range. The mass spectrum of the color-singlet pairs is asymptotically independent of the energy and production mechanism of the event and light clusters dominate. Due to a finite width of the mass distribution three possible cases have to be considered. If the cluster is too light to decay into two hadrons, it is taken to represent the lightest single hadron of its flavor and its mass is shifted to the appropriate value by an exchange of four momentum with a neighboring cluster in the jet. Massive clusters will be fragmented using an iterative fission model until the masses of the fission products fall below a defined threshold. Clusters with an adequate mass decay isotropically into pairs of hadrons. The probability for a certain hadron to be formed by a cluster is determined by different parameters like the cluster mass, or spin and masses of the hadrons.

\section{MC@NLO:}

MC@NLO [49] matches next-to-leading order calculations of a given QCD process with a Monte Carlo parton showering simulation based on HERWIG by using the subtraction method for NLO calculations. Hard emissions are treated as in NLO computations so that the NLO results for distributions are recovered upon an expansion in $\alpha_{s}$ while soft/collinear emissions are handled by the HERWIG MC simulation. The matching between the hard and the soft/collinear region is smooth. When MC generators generate events with real parton emission, kinematical configurations occur that are also taken into account by the NLO computation: the possibility of having the same kinematical configuration from the MC and from the NLO may lead to double counting. The problem is to merge the PS and the NLO description under avoidance of double-counting. MC@NLO uses the subtraction method [50] for NLO calculations to match the two elements, leading to a small amount of generated events with negative weights $(\sim 10 \%)$. In this way MC@NLO provides a fully exclusive event generation with hadronization according to the MC model, whereby the total exclusive rates are accurate to NLO computations.

\section{Matrix-element generators:}

Compared to the generators presented above, matrix-element generators do not contain parton showering methods. Given a process, they identify the relevant 
subprocesses and generate the corresponding amplitudes. The information about the contributing Feynman diagrams is then used to calculate cross sections and to obtain unweighted events at parton level (or tree level). Thus, the process is completed before hadronization. Since the event generation produces an output in the standard format, the parton-level sample can be passed on to a parton showering generator like PYthia or HERWIG to continue the evolution of partons into fully hadronized final states. For this analysis, certain background contributions are modeled by the matrix-element generator ALPGEN [51], whereby the parton showering is done with a HERWIG event generator. ALPGEN is dedicated to the study of multiparton hard processes in hard interactions, containing a description of $W$ boson production in association with heavy quark and light quark production, which is the main background contribution to the analyzed $t \bar{t}$ sample (see chapter 6.1).

MadEvent [52] is another multi-purpose tree-level event generator used to study the asymmetry in events, where the top pair and one additional jet are produced at parton level (see chapter 5.2.3). MadEvent is powered by the matrix-element generator MadGraph [53], which automatically generates the amplitudes for all the relevant subprocesses. This process-dependent information is packaged into MadEvent and a stand-alone code is produced to calculate the cross sections.

To obtain generated Monte Carlo event samples that are comparable to the measured data of the experiment, the interaction of the generated particles with the material of the CDF II detector is implemented by using the detector simulation package GEANT3 [?] and an analog signal processing and detector reconstruction is performed. The resulting Monte Carlo samples are then in the same format as data samples obtained from the experiment. The fact that the generated samples also contain full event information at parton level so that each particle at hadron level can be backtraced, offers several important opportunities. MC samples are used to understand better the influence of event selection and reconstruction effects on the data samples. They allow for an estimate of how many background events the data contain and how they are distributed. Finally we use several MC samples to study contributions of different systematic uncertainties. Table 4.1 gives an overview of the diverse Monte Carlo samples used in this analysis. The MC signal samples are all generated with a top mass of $175 \mathrm{GeV} / \mathrm{c}^{2}$. The background samples were generated with ALPGEN [51] and HERWIG, since ALPGEN contains a description of $W$ boson production in association with heavy quark and light quark production, appropriate to model parts of the background that contribute to the $t \bar{t}$ sample.

\subsection{Top-Pair Production at Monte Carlo Level}

Using the three signal Monte Carlo samples generated with PyTHIA, HERWIG, and MC@NLO, we study some properties of the top-pair production and decay. At the Tevatron the center-of-mass energy of the incoming partons in the partonic subsystem $\sqrt{\hat{s}}$ is described by the parton distribution functions (see chapter 1.3) of the colliding proton and antiproton. At a beam energy of $980 \mathrm{GeV}$ it is more likely to find a valence quark $(u, d)$ carrying a momentum fraction which is sufficient to pro- 


\begin{tabular}{|c|c|c|c|}
\hline Sample & Process & Generator & Comment \\
\hline \multicolumn{4}{|c|}{ Signal samples } \\
\hline ttopkl & $\overline{t \bar{t}}, m_{t}=175 \mathrm{GeV} / \mathrm{c}^{2}$ & PYThiA & \\
\hline ttopvh & $t \bar{t}, m_{t}=175 \mathrm{GeV} / \mathrm{c}^{2}$ & HERWIG & \\
\hline ptop10 & $t \bar{t}, m_{t}=175 \mathrm{GeV} / \mathrm{c}^{2}$ & $\mathrm{MC} @ \mathrm{NLO}$ & \\
\hline \multicolumn{4}{|c|}{ Background samples } \\
\hline ltop4n & $W(\rightarrow e \nu)+4 p$ & ALPGEN + HERWIG & \\
\hline ltop $4 \mathrm{~m}$ & $W(\rightarrow \mu \nu)+4 p$ & ALPGEN + HERWIG & \\
\hline ltop $2 b$ & $W(\rightarrow e \nu)+2 p+2 b$ & ALPGEN + HERWIG & \\
\hline ltop $5 b$ & $W(\rightarrow \mu \nu)+2 p+2 b$ & ALPGEN + HERWIG & \\
\hline \multicolumn{4}{|c|}{ Systematic samples } \\
\hline ttopel & $t \bar{t}, m_{t}=178 \mathrm{GeV} / \mathrm{c}^{2}$ & PYTHIA & diff. $m_{t}$ \\
\hline ttopvh & $t \bar{t}, m_{t}=175 \mathrm{GeV} / \mathrm{c}^{2}$ & HERWIG & diff. MC generator \\
\hline ttopbr & $t \bar{t}, m_{t}=178 \mathrm{GeV} / \mathrm{c}^{2}$ & PутніA & less ISR \\
\hline ttopdr & $t \bar{t}, m_{t}=178 \mathrm{GeV} / \mathrm{c}^{2}$ & PYthiA & more ISR \\
\hline ttopfr & $t \bar{t}, m_{t}=178 \mathrm{GeV} / \mathrm{c}^{2}$ & Pythia & less FSR \\
\hline ttopkr & $t \bar{t}, m_{t}=178 \mathrm{GeV} / \mathrm{c}^{2}$ & PythiA & more FSR \\
\hline ttopir & $t \bar{t}, m_{t}=178 \mathrm{GeV} / \mathrm{c}^{2}$ & PутніА & diff. PDF: MRST72 \\
\hline ttopjr & $t \bar{t}, m_{t}=178 \mathrm{GeV} / \mathrm{c}^{2}$ & PYтHIA & diff. PDF: MRST75 \\
\hline
\end{tabular}

Table 4.1: Used Monte Carlo samples in this analysis. In this chapter we study three different signal samples, generated by PYTHIA, HERWIG and MC@NLO each with a top-quark mass $m_{t}=$ $175 \mathrm{GeV} / \mathrm{c}^{2}$. The samples to model the different $t \bar{t}$ background contributions are obtained from ALPGEN and HERWIG. In the lower section are listed the used systematic samples. 
duce top-quark pairs than to find appropriate gluons. Thus, top-pair production is dominated by quark-antiquark annihilation with $85 \%$, while gluon fusion contributes only with $15 \%$. In figure 4.2 (a) the generated ratio between the two different production mechanism of the three Monte Carlo signal samples are compared. The left bin contains events produced via $q \bar{q}$ annihilation, in the right one events are filled with gluon fusion as production mechanism. The expected fraction is modeled very well by the NLO Monte Carlo generator MC@NLO. Compared to the NLO cross section of the different production processes, the LO cross sections contain less radiative contributions. Since the resulting reduction of the rate is smaller for quark-antiquark annihilation than for gluon fusion, the ratio of the cross sections is changed if only leading order contributions are considered. In both LO Monte Carlo signal samples the fraction of $q \bar{q}$ annihilation is about $94 \%$ and gluon fusion contributes with $6 \%$.

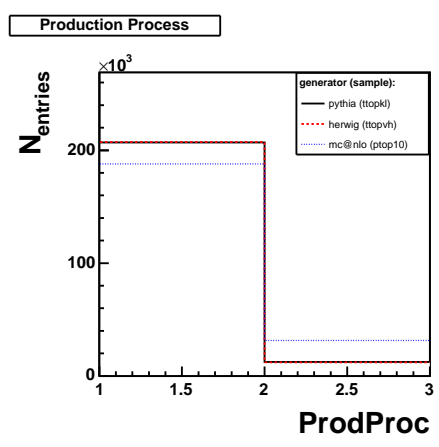

(a)

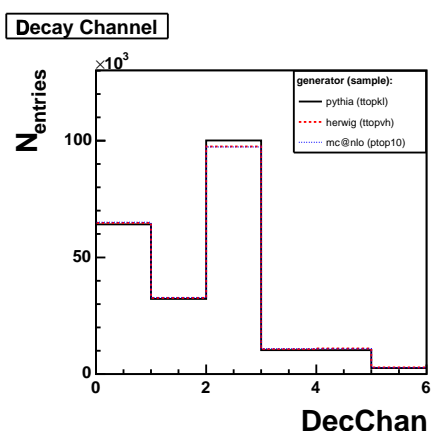

(b)

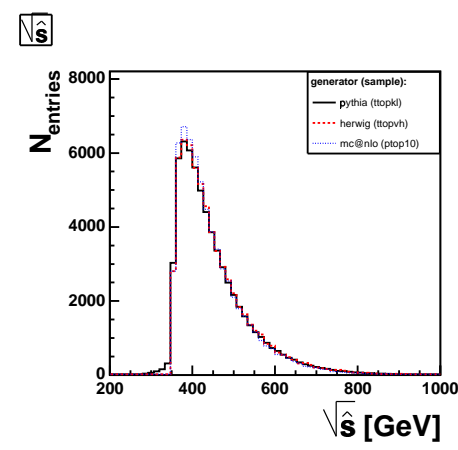

(c)

Figure 4.2: The rates of the two different production mechanisms of top-quark pairs (a) and the branching fractions for the possible decay channels (b) are compared for the used Monte Carlo signal samples generated with PYTHIA, HeRWIG, and MC@NLO. The left bin of the production process plot contains events produced via $q \bar{q}$ annihilation, the entries in the right bin represent gluon fusion. The coding for the label "DecChan" in the center plot is given in table 4.2. Plot (c) shows the distribution of $\sqrt{\hat{s}}$, the center-of-mass energy in the partonic rest frame. The distributions are all normalized to the HERWIG sample.

The expected theoretical branching ratios of top-quark pairs decaying into different final states were discussed in chapter 1.2. We check, whether the rates of the different channels are modeled correctly by the event generators. The rate of each decay channel is presented in figure 4.2 (b) for the three different Monte Carlo signal samples. The coding of the label "DecChan" can be found in table 4.2, where the different generated rates are compared to the expected theoretical prediction. The modeling of the rates in each of the six different decay channels is in good agreement with the expectation for all the used MC generators.

Since our measurement is done in the lepton+jets channel, corresponding to the decay channel " 0 " in the first line of table 4.2 , we consider in the following only events, where the top quarks decay in this channel. The center-of-mass energy in 


\begin{tabular}{c|c|cccc}
$W \rightarrow \ldots:$ & "DecChan" & $\mathrm{BR}^{\text {theo }}[\%]$ & $\mathrm{BR}^{\text {Pythia }}[\%]$ & $\mathrm{BR}^{\text {HERwig }}[\%]$ & $\mathrm{BR}^{\text {MC@ }}{ }^{\text {NLO }}[\%]$ \\
\hline \hline$q_{1}, \bar{q}_{2}, \ell, \nu$ & 0 & 29.63 & $29.23 \pm 0.02$ & $29.52 \pm 0.10$ & $29.62 \pm 0.06$ \\
$q_{1}, \bar{q}_{2}, \tau, \nu$ & 1 & 14.81 & $14.65 \pm 0.03$ & $14.87 \pm 0.08$ & $14.93 \pm 0.05$ \\
$q_{1}, \bar{q}_{2}, q_{3}, \bar{q}_{4}$ & 2 & 44.44 & $45.61 \pm 0.04$ & $44.44 \pm 0.11$ & $44.34 \pm 0.06$ \\
$\ell_{1}, \nu_{1}, \ell_{2}, \nu_{2}$ & 3 & 4.94 & $4.67 \pm 0.02$ & $4.88 \pm 0.05$ & $4.93 \pm 0.03$ \\
$\ell, \nu_{1}, \tau, \nu_{2}$ & 4 & 4.94 & $4.66 \pm 0.02$ & $4.99 \pm 0.05$ & $4.93 \pm 0.03$ \\
$\tau_{1}, \nu_{1}, \tau_{2}, \nu_{2}$ & 5 & 1.23 & $1.17 \pm 0.01$ & $1.28 \pm 0.02$ & $1.24 \pm 0.01$ \\
\hline \hline
\end{tabular}

Table 4.2: Expected theoretical branching ratios (BR) for the different decay channels of top-quark pairs. The rates modeled by the three Monte Carlo event generators are in good agreement with the expectation. The BRs of the lepton+jets channel ( $\ell$ represents electron or muon) are given in the first line corresponding to the label " 0 " of the parameter "DecChan" in figure 4.2 (b).

the partonic rest frame is given by the four-vectors $p_{i}$ of the incoming partons $i$ : $\sqrt{\hat{s}}=\sqrt{p_{1}^{2}+p_{2}^{2}}$. To produce top-quark pairs a center-of-mass energy corresponding to at least two times the top-quark mass $m_{t}=175 \mathrm{GeV} / \mathrm{c}^{2}$ is required. In figure 4.2 (c) the distribution of $\sqrt{\hat{s}}$ for the three studied $t \bar{t}$ signal samples are compared. The shapes of the different distributions are similar and show that most top-quark pairs are produced close to the threshold of $\sqrt{\hat{s}} \sim 350 \mathrm{GeV}$.

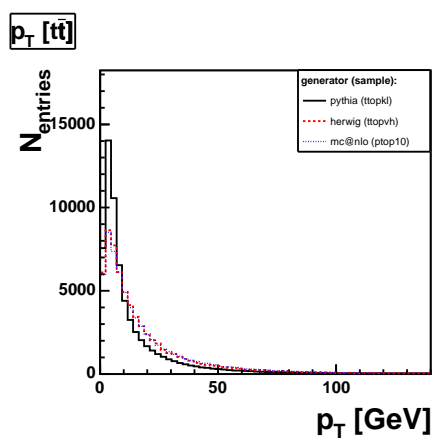

(a)

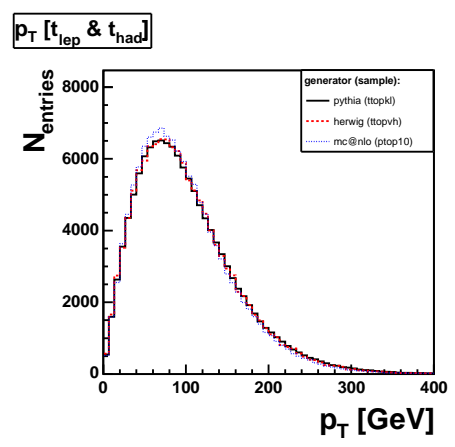

(b)

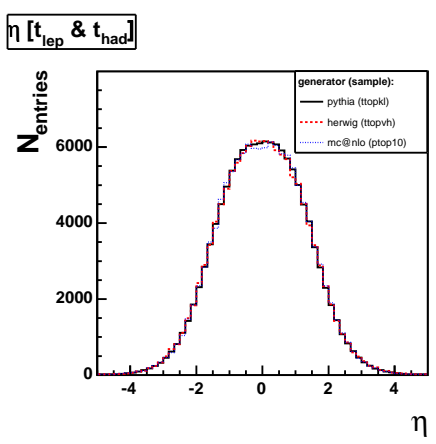

(c)

Figure 4.3: Properties of the produced top-quark pair generated by the different Monte Carlo signal samples. The transverse momentum $p_{T}(t \bar{t})$ of the sum of the top-quark four-vectors is plotted in (a). The distribution of the transverse momentum $p_{T}$ of all top quarks can be seen in (b). On the right hand side (c) the pseudo-rapidity distribution of all top quarks is shown. The distributions are always normalized to the sample generated by HERWIG.

In figure 4.3 some characteristics of the produced top-quark pairs are summarized. Plot (a) shows the transverse momentum of the sum of the top-quark fourvectors: $p_{T}(t \bar{t})=\sqrt{\left(p_{x, t}+p_{x, \bar{t}}\right)^{2}+\left(p_{y, t}+p_{y, \bar{t}}\right)^{2}}$. Since the top-quark pair is expected to be produced back-to-back, the transverse momentum of the sum should be zero. This is verified by the distributions of $p_{T}(t \bar{t})$ for each Monte Carlo signal sample. Due to gluon radiations of the top quarks, the values are distributed up to $50 \mathrm{GeV} / c$. 
A difference between the shapes for Pythia and HERWig/MC@NLO can be seen. In the case of PyтнiA, the distribution is sharper at zero, which indicates that the radiation is modeled slightly softer than by the other MC generators. The similar shapes for HERWIG and MC@NLO can be explained by the fact, that the parton showering used by MC@NLO is based on HERWIG. Thus, the slight differences in the shapes are due to the different implemented parton showering models.

In the center of figure 4.3 the transverse momentum distributions of both top quarks are plotted. The shapes produced by the different Monte Carlo generators are similar and most top quarks are produced with a large transverse momentum of about $80 \mathrm{GeV} / c$. The corresponding pseudo-rapidity distributions can be found in plot (c). The shapes of the different samples show the same symmetric distribution around zero, whereby most top quarks are produced in a region of $|\eta| \leq 2.0$. As already discussed, the top quarks decay before they hadronize. In the lepton+jets channel the charged lepton $(e, \mu)$ is the single parton, that is produced at parton level and can be observed more or less directly in the detector. The neutrino from the leptonic $W$ boson decay is assumed to be observable as missing transverse energy in the event. Thus, information about these two partons at generator level give a qualitative impression of the hardness of the event selection cuts applied to these two objects. Figure 4.4 (a, c) show the transverse momentum distributions of the charged lepton and the neutrino, figure (b, d) display the corresponding $\eta$ distributions. The shapes of $p_{T}$ and $\eta$ are similar for the charged lepton and the neutrino, the maximum of the transverse momentum is about $30 \mathrm{GeV} / c$ for the charged leptons, and about $40 \mathrm{GeV} / c$ for the neutrinos. Thus, the choice of the cuts at $p_{T, \ell, \nu} \geq 20$ $\mathrm{GeV} / c$ is an acceptable compromise. The pseudo-rapidity distributions on the right hand side are also both symmetric around zero but narrower than for the produced top quarks (figure 4.3). As can be seen from the $\eta$ plot of the charged lepton in figure 4.4 (b), the reduction of the event rate due to the selection cut of $|\eta| \leq 1.0$ for the charged lepton, which is determined by the calorimeter design, is quite substantial. Therefore, several efforts are made to expand the $\eta$-range of accepted charged leptons by including information from electromagnetic plug calorimeters [55]. Since additional backgrounds have to be considered in the forward region, these efforts are mainly driven by other analyses which elementarily depend on additional statistics [56]. 


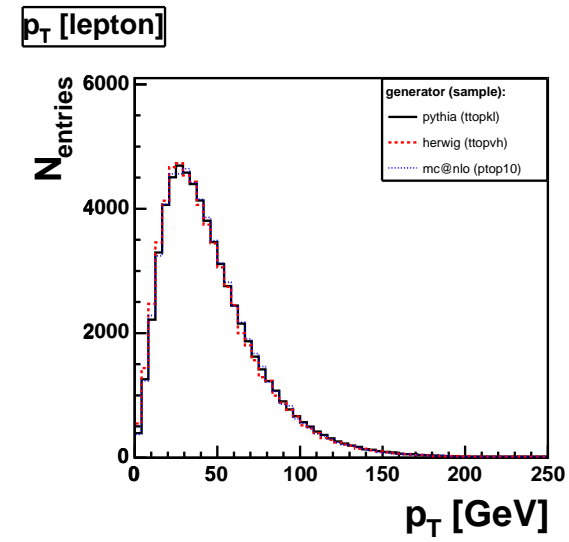

(a)

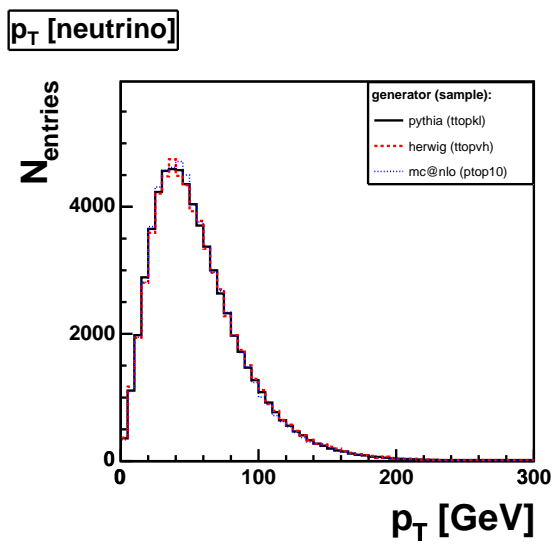

(c)

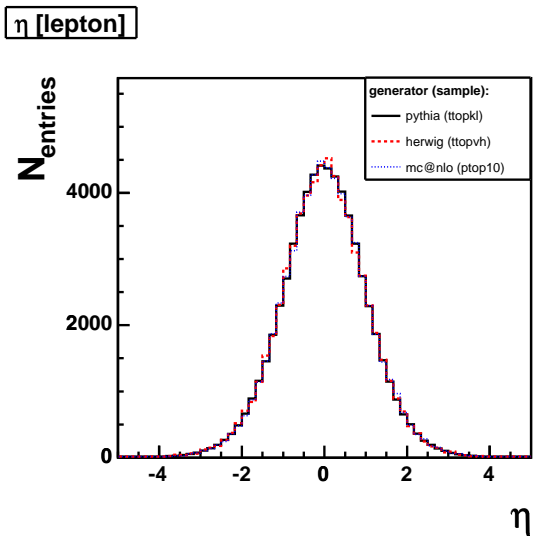

(b)

$\eta$ [neutrino]

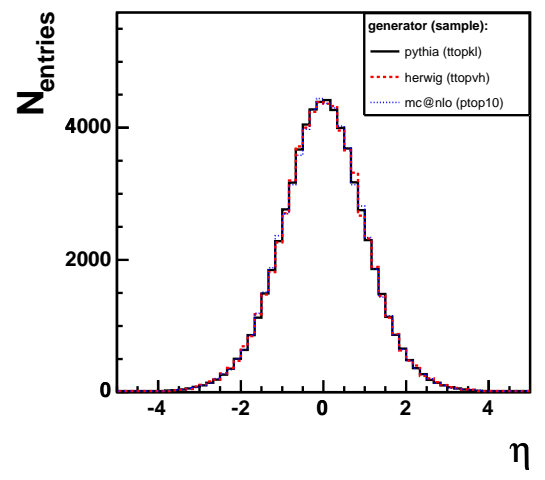

(d)

Figure 4.4: Transverse momentum and pseudo-rapidity of the charged lepton and neutrino from top-pair decays in the lepton+jets channel. The plots show the distributions at generator level for the three used Monte Carlo event generators. The rates are normalized to the HERWIG sample. 


\section{Chapter 5}

\section{Study of Asymmetry in Top-Pair Production}

In this chapter several Monte Carlo based studies of the asymmetry in top pair production are presented in detail. First, we investigate the influence of the event selection on the asymmetry $A$. This study yields a strong dependence of $A$ on the number of observed jets in an event. Since a jet multiplicity greater than four in $t \bar{t}$ events is mainly due to additional gluon radiation, the result of the selection study prompts further investigation of the influence of gluon radiation on the top-quark rapidity. The findings of these observations point out, that different contributions to a measured asymmetry in top-pair production have to be distinguished.

\subsection{Influence of the Event Selection on the Asym- metry}

Preparing for the measurement of the asymmetry $A\left(\Delta y \cdot Q_{l}\right)$ in top-pair production, we conduct some studies at parton level on the base of three signal Monte Carlo samples generated with PythiA, HeRWig, and MC@NLO. To calculate the rapidity difference $\Delta y \cdot Q_{l}=\left(y_{\text {tlep }}-y_{\text {thad }}\right) \cdot Q_{l}$ the generated (true) four-vectors of the produced top quarks, which are available in Monte Carlo samples, are used. The distributions of the rapidity difference for the three different generators, normalized to the HERWIG sample, are compared in figure 5.1. The resulting asymmetry values in these generated distributions are listed in table 5.1. At leading-order, calculations of the cross sections for top-pair production via quark-antiquark annihilation, $\sigma_{q \bar{q} \rightarrow t \bar{t}}$ and $\sigma_{q \bar{q} \rightarrow \bar{t}}$, are equal. Thus, no asymmetry is expected in the rapidity distributions of Pythia and HeRWIG. The MC@NLO sample should yield the expected value of about 5\%. As can be seen in table 5.1, the theoretical expectations are well confirmed by all the considered MC signal samples.

In the next step, the lepton+jets event selection discussed in section 3.1 is applied to each of the three MC signal samples. The asymmetry is again calculated by using the generated top-quark four-vectors, with the difference, that the considered sample only contains those events, that satisfy all event selection criteria. The resulting 


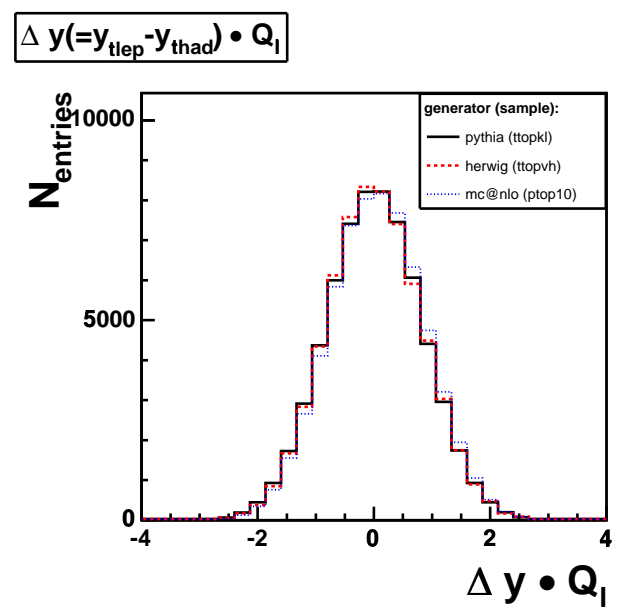

Figure 5.1: The truth distribution of $\Delta y \cdot Q_{l}$ for leading order (PyнthiA, HERWIG) and next-toleading order event generators (MC@NLO). The shapes are normalized to the HERWIG distribution.

values of $A^{\text {sel }}\left(\Delta y \cdot Q_{l}\right)$ for the different selected MC signal samples are listed in table 5.1 in the right column. Compared to the values of $A^{g e n}\left(\Delta y \cdot Q_{l}\right)$ in the generated inclusive samples, the asymmetry is shifted by about $3 \%$ points into the negative direction when the event selection is applied.

Since the amplitude of this shift is of the same order as the effect we intend to measure, further studies are necessary to understand the origin of this behavior. Our investigation is organized as follows. We start at parton level, where some basic selection cuts are applied on the final state partons accessible in the MC samples. At this, we distinguish between the effect of selection cuts and of isolation cuts on the asymmetry. The selection cuts set limits on the transverse momenta and the pseudorapidities of the charged lepton, neutrino, and the four quarks. The isolation cuts are in the form of a restriction of the distance $\Delta R=\sqrt{(\Delta \eta)^{2}+(\Delta \Phi)^{2}}$ between two particles in the $\eta-\phi$ plane. Secondly, a jet clustering at hadron level is implemented, thus the base of the study is closer to a final state configuration like we observe in measured data. Finally, with the results of these intermediate steps, the influence of the full lepton+jets event selection on the top-quark rapidity distributions will be discussed.

\begin{tabular}{cc|ccc}
\multicolumn{2}{c|}{ Generator } & $A^{\exp }\left(\Delta y \cdot Q_{l}\right)[\%]$ & $A^{\text {gen }}\left(\Delta y \cdot Q_{l}\right)[\%]$ & $A^{\text {sel }}\left(\Delta y \cdot Q_{l}\right)[\%]$ \\
\hline \hline LO: & HERWIG & 0.0 & $0.1 \pm 0.4$ & $-2.7 \pm 1.3$ \\
& PYTHIA & 0.0 & $0.3 \pm 0.1$ & $-2.9 \pm 0.5$ \\
NLO: & MC@NLO & $(4 \ldots 6)$ & $4.8 \pm 0.3$ & $2.6 \pm 0.9$ \\
\hline \hline
\end{tabular}

Table 5.1: The Monte Carlo truth values of the asymmetry $A^{\text {gen }}$ (center column) are consistent with the theoretical expectation (left column). The selected Monte Carlo values of the asymmetry $A^{\text {sel }}$ (right column) are shifted into the negative direction. The values are presented for the three different event generators, each given with the statistical errors $\sigma_{\text {stat }}$. 


\subsubsection{Influence of Different Cuts at Parton Level (PL)}

Several selection and isolation cuts are applied to the partons emerging directly from the decaying $t$ quark and $W$ bosons. The cuts are chosen to be comparable to the lepton+jets event selection described in chapter 3.1. The different cuts and their effects on $A$ for the three different Monte Carlo event generators are listed in detail in table 5.2. The upper section contains the selection cuts on $p_{T}$ and $\eta$ followed by

Influence of various selection cuts applied at parton level:

\begin{tabular}{|c|c|c|c|c|c|c|}
\hline \multirow[b]{2}{*}{ ut } & \multicolumn{2}{|c|}{$\mathrm{MC} @ \mathrm{NLO}$} & \multicolumn{2}{|c|}{ РүтнIA } & \multicolumn{2}{|c|}{ HERWIG } \\
\hline & $A[\%]$ & $\Delta A[\%]$ & $A[\%]$ & $\Delta A[\%]$ & $A[\%]$ & $\Delta A[\%]$ \\
\hline truth & $4.83 \pm 0.28$ & & $-0.10 \pm 0.30$ & & $0.15 \pm 0.39$ & \\
\hline$p_{T}^{\nu}>15 \mathrm{GeV}$ & $4.92 \pm 0.28$ & 0.09 & $-0.14 \pm 0.30$ & -0.04 & $0.18 \pm 0.40$ & 0.03 \\
\hline$p_{T}^{l e p}>20 \mathrm{GeV}$ & $4.85 \pm 0.30$ & 0.02 & $-0.11 \pm 0.33$ & -0.01 & $0.10 \pm 0.43$ & -0.05 \\
\hline$\left|\eta^{l e p}\right|<1.0$ & $4.26 \pm 0.33$ & -0.57 & $-0.39 \pm 0.36$ & -0.29 & $-0.13 \pm 0.47$ & -0.28 \\
\hline$p_{T}^{b_{\text {bep }}}>15 \mathrm{GeV}$ & $4.97 \pm 0.28$ & 0.14 & $-0.03 \pm 0.30$ & 0.07 & $0.26 \pm 0.40$ & 0.11 \\
\hline$\eta^{b_{\text {lep } p}} \mid<2.0$ & $4.90 \pm 0.28$ & 0.07 & $-0.01 \pm 0.31$ & 0.09 & $0.25 \pm 0.41$ & 0.10 \\
\hline$p_{T}^{b_{\text {had }}}>15 \mathrm{GeV}$ & $4.85 \pm 0.28$ & 0.02 & $-0.06 \pm 0.30$ & 0.04 & $0.14 \pm 0.40$ & -0.01 \\
\hline$\left|\eta^{b_{\text {had }}}\right|<2.0$ & $4.68 \pm 0.28$ & -0.15 & $-0.01 \pm 0.31$ & 0.09 & $0.15 \pm 0.40$ & - \\
\hline$p_{T}^{Q 1}>15 \mathrm{GeV}$ & $4.87 \pm 0.28$ & 0.04 & $-0.23 \pm 0.31$ & 0.13 & $-0.03 \pm 0.40$ & -0.18 \\
\hline$\left|\eta^{Q 1}\right|<2.0$ & $4.72 \pm$ & -0.11 & $-0.10 \pm 0.31$ & - & $0.24 \pm 0.40$ & 0.09 \\
\hline$p_{T}^{Q 2}>15 \mathrm{GeV}$ & $4.72 \pm 0.29$ & -0.11 & $-0.17 \pm 0.31$ & -0.07 & $0.02 \pm 0.41$ & -0.13 \\
\hline$\left|\eta^{Q 2}\right|<$ & $4.73 \pm 0.28$ & -0.10 & $-0.11 \pm 0.31$ & -0.01 & $0.00 \pm$ & -0.15 \\
\hline$\Delta R_{\text {lepb }}$ lep $>0.4$ & $4.92 \pm 0.27$ & $\overline{0.09}$ & \pm 0.30 & -0.02 & $0.11 \pm 0.40$ & $\overline{-0.04}$ \\
\hline$\Delta R_{l e p b_{\text {had }}}>0.4$ & $4.92 \pm 0.28$ & 0.09 & $-0.07 \pm 0.30$ & 0.03 & $0.15 \pm 0.40$ & - \\
\hline$\Delta R_{l e p Q 1}>0.4$ & $4.82 \pm 0.28$ & -0.01 & $-0.17 \pm 0.30$ & -0.07 & 40 & 0.01 \\
\hline$\Delta R_{\text {lepQ2 }}>0.4$ & 4.8 & 0.05 & \pm 0 & 0.10 & 40 & 0.02 \\
\hline$\Delta R_{Q 1 Q 2}>0.4$ & 4. & - & $-0.10 \pm 0.30$ & - & 40 & - \\
\hline$\Delta R_{Q 1 b_{l e p}}>0.4$ & $4.96 \pm$ & 0.13 & -0 . & 0.05 & \pm & 0.08 \\
\hline$\Delta R_{Q 1 b_{\text {had }}}>0.4$ & $4.8:$ & - & \pm 0.30 & 0.01 & 40 & -0.01 \\
\hline$\Delta R_{Q 2 b_{l e p}}>0.4$ & \pm 0.28 & 0.07 & -0 & 0.04 & 40 & -0.02 \\
\hline$\Delta R_{Q 2 b_{\text {had }}}>0.4$ & $4.86 \pm 0.27$ & 0.03 & $-0.11 \pm 0.30$ & -0.01 & \pm & 0.08 \\
\hline$\Delta R_{b_{l e p} b_{\text {had }}}>0.4$ & $4.88 \pm 0.28$ & 0.05 & $-0.10 \pm 0.30$ & - & $0.30 \pm 0.40$ & 0.15 \\
\hline all $p_{T}$, & $\overline{4}$ & 0.5 & $-0.62 \pm$ & -0.52 & $-0.36 \pm$ & -0.51 \\
\hline & & & 2 & 0.15 & 42 & 0.26 \\
\hline all cuts $(P L)$ & $4.82 \pm 0.47$ & 0.01 & $-0.36 \pm 0.51$ & -0.26 & $-0.17 \pm 0.68$ & -0.32 \\
\hline
\end{tabular}

Table 5.2: The effect of different selection and isolation cuts on the asymmetry $A$ along with the statistical errors. The resulting values are compared to the generated ones and the deviation $\Delta A$ is given. In the first two sections the cuts are applied separately at parton level, the last section shows the results for combined applications. We compare three different event generators.

the isolation cuts on $\Delta R$, which are all applied separately. There is no single cut which has a sizeable effect on $A$, except for the $\left|\eta^{l e p}\right|$ cut in line 4 . Restricting the $\eta$ range of the charged lepton to the central region slightly reduces the asymmetry value by about a half percentage point in the NLO Monte Carlo sample. In the last section of table 5.2 we show the effects of combining several cuts.

The simultaneous application of all selection cuts on $p_{T}$ and $\eta$ indicates a small systematic effect on $A$. In the MC@NLO sample the asymmetry is reduced by about 


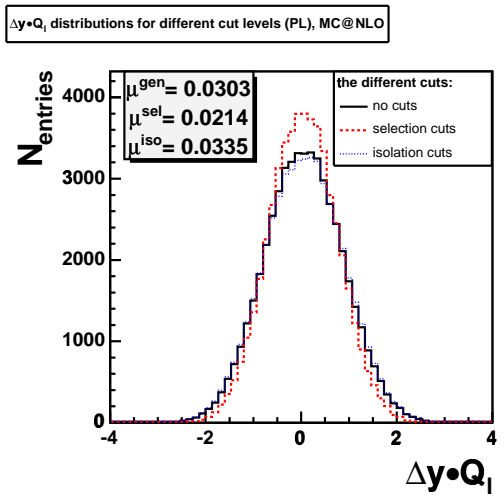

(a)

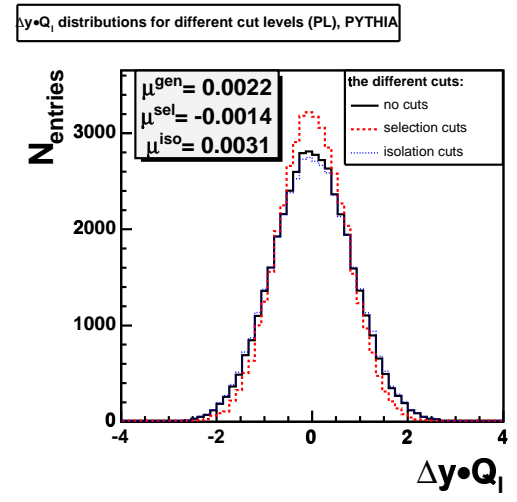

(b)

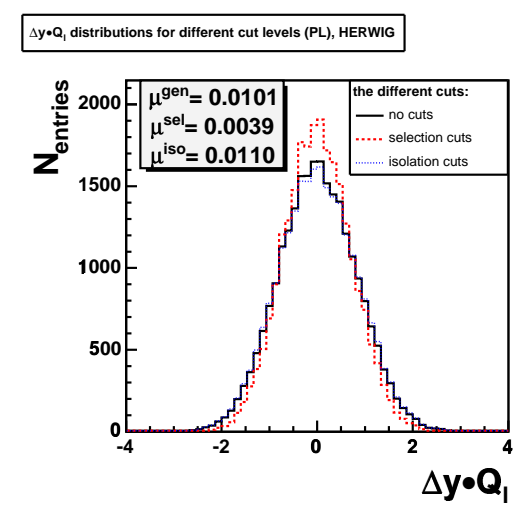

(c)

Figure 5.2: The distributions of $\Delta y \cdot Q_{l}$ for different cut applications at parton level and for the three Monte Carlo event generators: (a) MC@NLO, (b) PYTHiA, and (c) HERWiG. The black solid line shows that distribution without applying any cuts, while for the dashed red line only the selections cuts on $p_{T}$ and $\eta$ are applied and for the dotted blue line only the isolation cuts on $\Delta R$. The corresponding means are displayed in the boxes.

0.6 percentage points. However, this is still on the level of the statistical uncertainty. A similar negative tendency is observed for the LO Monte Carlo samples PythiA and HERWIG. On the other hand, the restrictions of $\Delta R$ slightly enhance the asymmetry. Thus, the asymmetry values of all Monte Carlo samples are compatible with the corresponding generated ("truth") values if applying all cuts simultaneously (line "all cuts" of table 5.2).

In figure 5.2 the distributions of the rapidity difference $\Delta y \cdot Q_{l}$ before and after the different cuts are shown for each of the three generators. Application of selection cuts means that all $p_{T}$ and $\eta$ cuts are simultaneously made, the isolation cuts include all $\Delta R$ cuts, corresponding to the last section of 5.2. The means of 
the distributions, representing the tendency of $A$, confirm the different influence of selection and isolation cuts on the asymmetry.

\subsubsection{Influence of Different Cuts at Jet Level (JL)}

Since the previous study at parton level gives no explanation for the asymmetry offset observed after applying the lepton+jets event selection, we modify the base of the study. To reach a more realistic scenario than the partonic one a jet clustering is implemented at hadron level. In this study the $K_{T}$ algorithm [57] is used to build the jets. The final state is now characterized by the charged lepton and the neutrino from the leptonically decaying $W$ boson and at least four jets due to the hadronically decaying $W$ boson, the $b$ quarks from the top-quark decays, or potential hard radiation. Similar selection and isolation cuts as before are applied to those final-state objects. The number of jets, $N_{\text {jets }}$, is required to be greater than or equal to 4 . The $K_{T}$ settings are chosen in a way that the jets are clustered within a cone radius of $R \leq 0.4$ in the $\eta-\phi$ plane. The lower cut-off $d_{\text {min }}^{\text {cut }}$ is set to 0.0065 which corresponds approximately to a minimal transverse jet energy $E_{T}^{\text {jet }}$ of about $15 \mathrm{GeV}$. The particular effects on the asymmetry $A$ in the three Monte Carlo samples are listed in table 5.3 which is divided into the same sections as above.

Influence of various selection cuts applied at jet level:

\begin{tabular}{|c|c|c|c|c|c|c|}
\hline \multirow{2}{*}{$\begin{array}{l}N_{\text {jets }} \geq 4 \\
\quad \text { cut }\end{array}$} & \multicolumn{2}{|c|}{$\mathrm{MC} @ \mathrm{NLO}$} & \multicolumn{2}{|c|}{ РYTHIA } & \multicolumn{2}{|c|}{ HERWIG } \\
\hline & $A[\%]$ & $\Delta A[\%]$ & $A[\%]$ & $\Delta A[\%]$ & $A[\%]$ & $\Delta A[\%]$ \\
\hline truth & $3.87 \pm 0.30$ & & $-1.89 \pm 0.33$ & & $-1.15 \pm 0.43$ & \\
\hline$p_{T}^{\nu}>15 \mathrm{GeV}$ & $3.95 \pm 0.30$ & 0.08 & $-1.95 \pm 0.34$ & -0.06 & $-1.20 \pm 0.44$ & -0.05 \\
\hline$p_{T}^{l e p}>20 \mathrm{GeV}$ & $3.85 \pm 0.35$ & -0.02 & $-1.89 \pm 0.36$ & - & $-1.23 \pm 0.47$ & -0.08 \\
\hline$\left|\eta^{l e p}\right|<1.0$ & $3.48 \pm 0.32$ & -0.39 & $-2.07 \pm 0.40$ & -0.18 & $-1.28 \pm 0.51$ & -0.13 \\
\hline$p_{T}^{J e t 1}>15 \mathrm{GeV}$ & $3.87 \pm 0.29$ & - & $-1.89 \pm 0.33$ & - & $-1.15 \pm 0.43$ & - \\
\hline$\left|\eta^{\text {Jet } 1}\right|<2.0$ & $3.83 \pm 0.29$ & 0.04 & $-1.89 \pm 0.33$ & - & $-1.14 \pm 0.43$ & 0.01 \\
\hline$p_{T}^{J e t 2}>15 \mathrm{GeV}$ & $3.87 \pm 0.29$ & - & $-1.89 \pm 0.33$ & - & $-1.15 \pm 0.43$ & - \\
\hline$\left|\eta^{\text {Jet } 2}\right|<2.0$ & $3.81 \pm 0.30$ & 0.06 & $-1.92 \pm 0.33$ & -0.03 & $-1.22 \pm 0.43$ & -0.07 \\
\hline$p_{T}^{J e t 3}>15 \mathrm{GeV}$ & $3.87 \pm 0.29$ & - & $-1.89 \pm 0.33$ & - & $-1.15 \pm 0.43$ & - \\
\hline$\left|\eta^{\text {Jet } 3}\right|<2.0$ & $3.67 \pm 0.30$ & -0.20 & $-1.96 \pm 0.33$ & -0.07 & $-1.34 \pm 0.43$ & -0.19 \\
\hline$p_{T}^{J e t 4}>15 \mathrm{GeV}$ & $3.88 \pm 0.29$ & 0.01 & $-1.91 \pm 0.33$ & 0.02 & $-1.18 \pm 0.43$ & -0.03 \\
\hline$\left|\eta^{\text {Jet } 4}\right|<2.0$ & $3.48 \pm 0.30$ & -0.39 & $-2.05 \pm 0.34$ & -0.16 & $-1.61 \pm 0.44$ & -0.46 \\
\hline$\Delta R_{\text {lepJet } 1}>0.4$ & $3.90 \pm 0.30$ & 0.03 & $-1.83 \pm 0.33$ & $\overline{0.06}$ & $-1.18 \pm 0.43$ & -0.03 \\
\hline$\Delta R_{\text {lepJet } 2}>0.4$ & $95 \pm 0.30$ & 0.08 & $-1.95 \pm 0.33$ & -0.06 & $-1.10 \pm 0.43$ & 0.05 \\
\hline$\Delta R_{\text {lepJet } 3}>0.4$ & $3.91 \pm 0.30$ & 0.04 & $-1.86 \pm 0.33$ & 0.03 & $-1.10 \pm 0.43$ & 0.05 \\
\hline$\Delta R_{\text {lepJet } 4}>0.4$ & $3.96 \pm 0.30$ & 0.09 & $-1.91 \pm 0.33$ & -0.02 & $-1.18 \pm 0.43$ & -0.03 \\
\hline all $p_{T}, \eta(\mathrm{JL})$ & $2.84 \pm 0.42$ & -1.03 & $-2.79 \pm($ & -0.90 & $-2.17 \pm 0.61$ & -1.02 \\
\hline all $\Delta R(\mathrm{JL})$ & $4.11 \pm 0.30$ & 0.24 & $-1.85 \pm 0.34$ & 0.04 & $-1.12 \pm 0.44$ & 0.03 \\
\hline all cuts $(\mathrm{JL})$ & $3.04 \pm 0.43$ & -0.83 & $-2.72 \pm 0.48$ & -0.83 & $-2.12 \pm 0.63$ & -0.97 \\
\hline
\end{tabular}

Table 5.3: The effect of different selection and isolation cuts on the asymmetry $A$. The resulting values are compared to the generated one in the inclusive sample and the deviation $\Delta A$ is given. In the first two sections the cuts are applied separately at jet level, the last section shows the results for combined applications. The number of jets in an event is required to be greater or equal to four. 


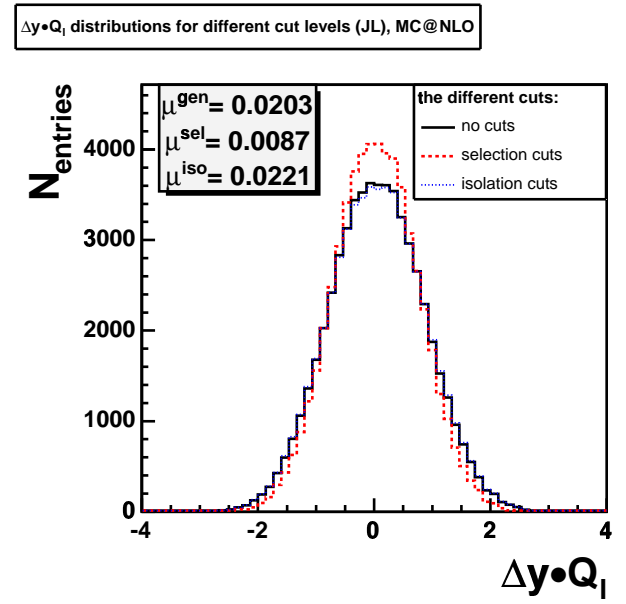

(a)

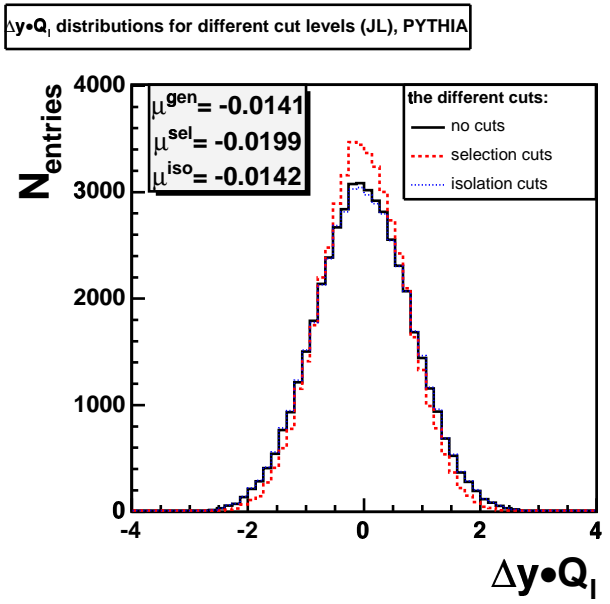

(b)

$\Delta y \cdot Q_{1}$ distributions for different cut levels (JL), HERWIG

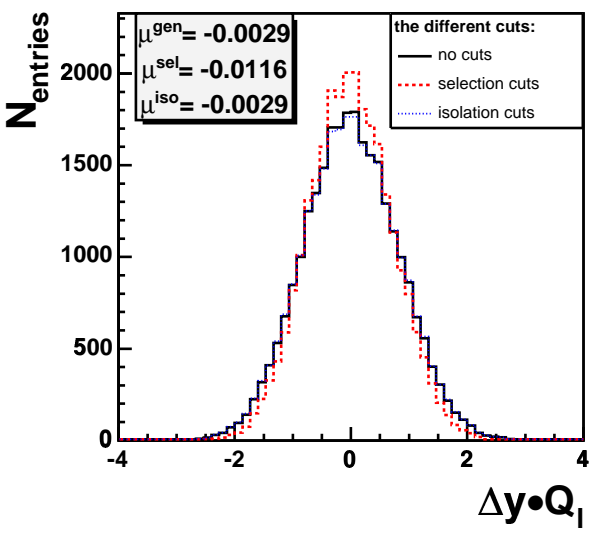

(c)

Figure 5.3: The distributions of $\Delta y \cdot Q_{l}$ for different cut applications at jet level and for the three Monte Carlo event generators: (a) MC@NLO, (b) PyThiA, and (c) HERWig. The black solid line shows that distribution without applying any cuts, while for the red line only the selections cuts on $p_{T}$ and $\eta$ are applied and for the blue line only the isolation cuts on $\Delta R$. The corresponding means are displayed in the boxes. 
If we consider only events with at least four jets but apply no further cuts, the asymmetry is already shifted from $A^{\mathrm{MC} @ \mathrm{NLO}}=4.83 \%$ for all events to $A^{\mathrm{MC} @ \mathrm{NLO}}=$ $3.87 \%$ (see line 1 indicated with "truth" in table 5.3). Furthermore, a significant shift is observable in the lower section of the table: the value of the asymmetry is reduced from original $A^{\mathrm{MC} @ \mathrm{NLO}}=4.83 \%$ to $A^{\mathrm{MC} @ \mathrm{NLO}}=3.04 \%$ if we require at least four jets and apply simultaneously all cuts at jet level. This study identifies the jet selection as the main issue responsible for the negative shift of the asymmetry. However, this reduction describes not entirely the observed shift of about three percentage points into the negative direction if the full lepton + jets event selection is applied. However, this study yields the jet selection as a main issue responsible for the negative shift of the asymmetry. Figure 5.3 shows a comparison of the $\Delta y \cdot Q_{l}$ distributions if the combined selection $\left(p_{T}, \eta\right)$ and isolation $(\Delta R)$ cuts at jet level are made. The means of the distributions stay independent of the isolation cuts, whereas the selection cuts on the transverse momenta and pseudo-rapidities of the particles and jets shift the means slightly into the negative direction.

The study at jet level offers the possibility to check the exclusive asymmetry in different jet bins, which means that the events are classified by the numbers of observed jets. By doing this jet-bin study an unexpected effect occurs: the Monte Carlo true value of $A$ shows a linear dependence on the required number of jets in the event. As can be seen in table 5.4 this dependence is valid for each of the three generators. The next-to-leading order asymmetry shows up only in the MC@NLO sample, but the linear dependence of $A$ on the number of jets occurs with the same strength also in the samples generated with PYTHIA and HERWIG.

\begin{tabular}{c|ccccc}
$N_{\text {jets }}=$ & 4 & 5 & 6 & 7 & 8 \\
\hline \hline$A(\mathrm{MC} @ \mathrm{NLO})[\%]$ & 9.0 & 2.8 & -4.5 & -9.3 & -18.9 \\
$A(\mathrm{HERWIG})[\%]$ & 5.5 & -2.8 & -12.4 & -23.0 & -25.2 \\
$A(\mathrm{PYTHIA})[\%]$ & 6.4 & -4.5 & -16.8 & -26.1 & -35.4 \\
\hline \hline
\end{tabular}

Table 5.4: Values of the asymmetry $A$ depending on the number of jets required in the events for the three different generators. No selection cuts are applied except for the jet classification.

Figure 5.4 visualizes the linear character of the jet-bin effect for the next-toleading order sample generated with the MC@NLO event generator. The values of $A$ are plotted against the number $N_{\text {jets }}$ of jets. No selection cuts except for the jet classification are applied. In the four-jets bin $\left(N_{\text {jets }}=4\right)$ the exclusive asymmetry is raised to a value of $9 \%$, which is almost twice the expected value of $A$ in inclusive next-to-leading order calculations. If the number of required jets is increased the exclusive asymmetry is reduced linearly down to sizeable negative values. The linear dependence of the asymmetry value on the number of measured jets remains if all the selection and isolation cuts mentioned in table 5.3 are applied. The corresponding plots are shown in figure 5.5. The application of all cuts only changes slightly the slope and the intersection point of the linear fit function, see table 5.5, but the linear dependence still holds.

The influence of the hardness of the clustered jets on the observed linear effect 


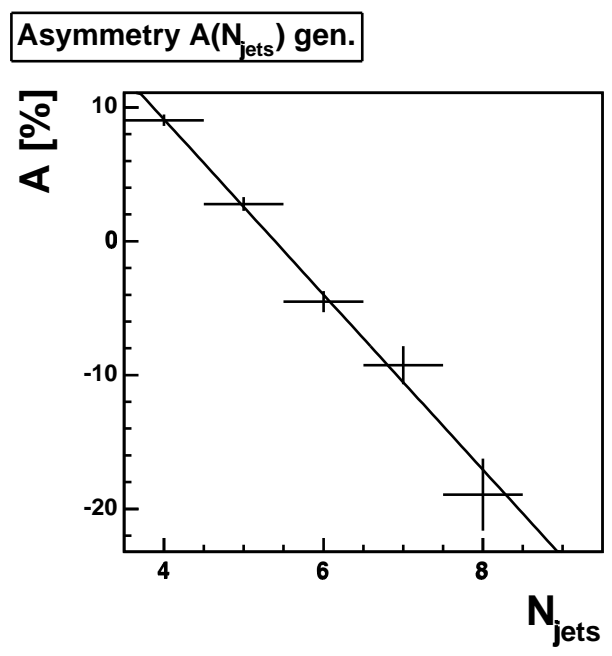

Figure 5.4: The asymmetry as a function of $N_{\text {jets }}$ for the MC@NLO sample (ptop10): a linear fit function describes the trend very well. No selection cuts are applied except for the jet classification demanding $E_{T}^{j e t} \geq 15 \mathrm{GeV}$ and $|\eta| \leq 2.0$.

\begin{tabular}{c||ccc} 
& MC@NLO & PYTHIA & HERWIG \\
\hline \hline$A^{\text {truth }[\%]}$ & 4.83 & -0.10 & 0.14 \\
slope & -6.5 & -11.0 & -9.3 \\
ip & 38 & 50 & 43 \\
\hline$A^{\text {allcuts }(J L)}[\%]$ & 3.04 & -2.72 & -2.12 \\
slope & -6.2 & -10.5 & -7.9 \\
ip & 36 & 47 & 36 \\
\hline \hline
\end{tabular}

Table 5.5: The parameters of the linear fit functions (slope and intersection point "ip") for the different event generators. The upper part of the table shows the value if the Monte Carlo truth is considered. The lower part contains the values if all the cuts at jet level are applied. 


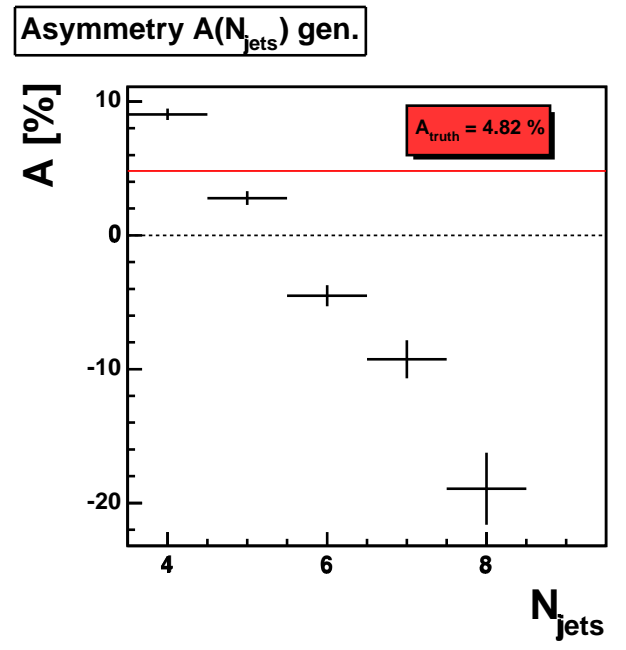

(a)

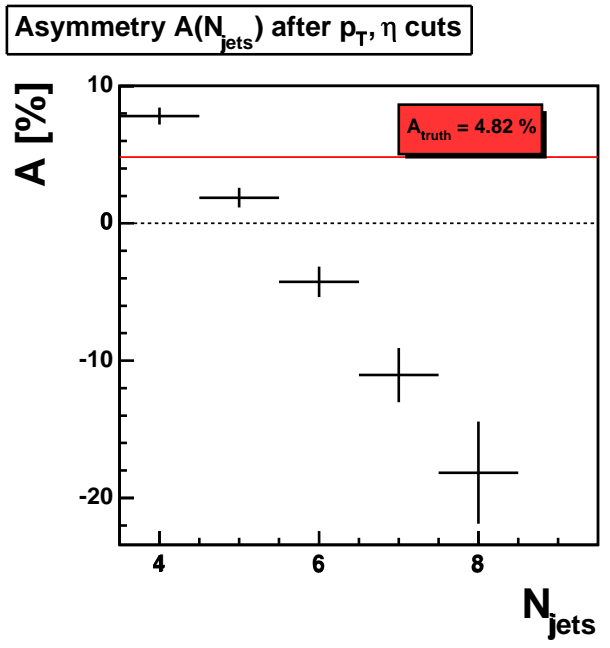

(b)

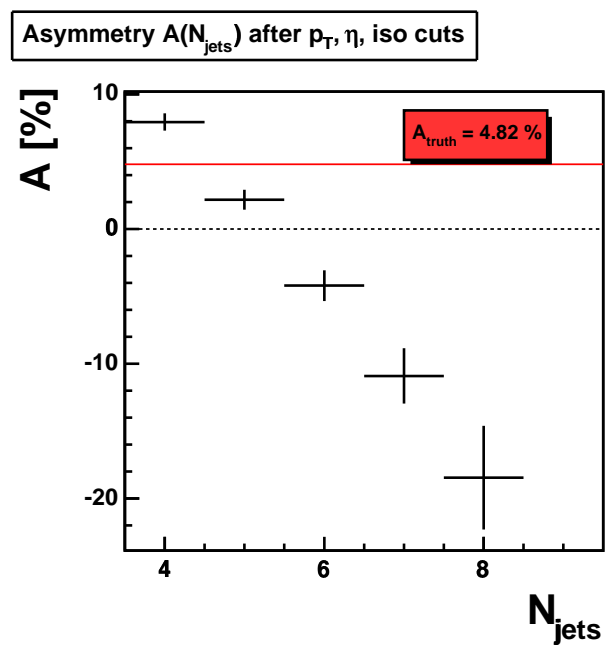

(c)

Figure 5.5: The MC@NLO Monte Carlo asymmetry for different jet numbers: no cuts except for the jet multiplicity requirement is made (a), only selection cuts are applied (c) and the distribution if all cuts are made (b): a linear dependence is observable independent on whether selection cuts are applied or not. The red line represents the value of the inclusive asymmetry for the Monte Carlo truth distribution, i.e. no jet requirement is made and no cuts are applied; the same value is displayed in the red colored box. 
is investigated by varying the lower cut-off $d_{\min }^{\text {cut }}$ of the $K_{T}$ algorithm. The effect of these parameter modifications on the asymmetry $A\left(\Delta y \cdot Q_{l}\right)$ is exemplarily shown for the leading order HERWIG Monte Carlo sample in figure 5.6. We have verified the same behavior for the PYthia and MC@NLO Monte Carlo samples.

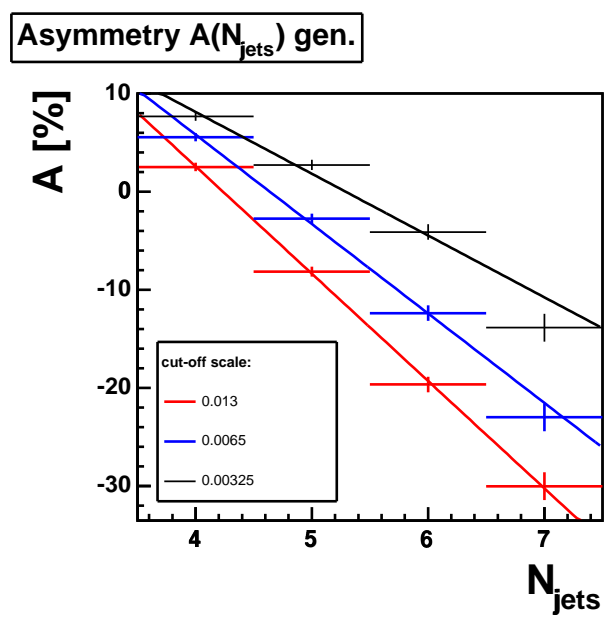

Figure 5.6: The asymmetry as a function of the number of jets for three different cut-off scales: the harder the clustered jets, the steeper the linear trend. The leading order HERWIG Monte Carlo sample is used.

Fitting a straight line to each of the $N_{\text {jets }}$ distributions yields:

$$
\begin{aligned}
& A=-6.88 \cdot N_{\text {jets }}+36.58 \text { (softer jets) } \\
& A=-9.28 \cdot N_{\text {jets }}+43.26 \text { (unchanged setting) } \\
& A=-10.89 \cdot N_{\text {jets }}+46.10 \text { (harder jets) }
\end{aligned}
$$

As can be seen in this figure the slope of the linear fit function decreases if the parameter is halved to $d_{\min }^{c u t}=0.00325$, otherwise if it is doubled to $d_{m i n}^{c u t}=0.013$ the slope gets steeper. This can be explained by the fact that a modification of the cut-off varies the jet multiplicity in the events. This modification leads to a changed population of the different jet bins. Some events observed in a certain jet bin, migrate to a lower jet bin in the case of a harder jet clustering cut-off. Thus, the asymmetry in each jet bin changes. The higher the cut-off scale, the steeper the effect on the asymmetry with the number of jets.

\subsubsection{Influence of the CDF Standard Lepton + Jets Event Selection on $A\left(\Delta y \cdot Q_{l}\right)$}

The previous Monte Carlo study at jet level already indicates the main reason for the event selection offset, namely the treatment of jets by the selection. We now aim to examine the influence of the full lepton + jets event selection used in this analysis 
on the inclusive asymmetry $A\left(\Delta y \cdot Q_{l}\right)$ to explain conclusively the observed selection shift.

To clarify the effect of different cuts, the several elements of the lepton+jets event selection were combined to three categories, each including cuts that affect another class of physical objects. We distinguish between cuts and requirements

- affecting CEM electrons

- on CMUP/CMX muons

- concerning the clustered jets

In the first line of table 5.6 indicated with "no cuts" one can find the asymmetry values of the original Monte Carlo samples before the full event selection. The values are equal to those presented in table 5.1 (middle column). The second line of table 5.6 ("full selection") shows the values of $A$ after the full event selection is applied, corresponding to the values in the right column of table 5.1. Since we limited the number of considered events in the PYTHIA sample to 500.000 the asymmetry values before and after the event selection differ insignificantly from the ones presented in table 5.1. In a first step we consider only events in which the charged lepton is a CEM electron and apply only those cuts concerning this lepton. We repeat the procedure for central muons (CMUP, CMX) and only the corresponding muon cuts are made. The asymmetry values for both cases and for each of the three Monte Carlo event generators can be found in table 5.6. The results indicate that the selection of the charged lepton induces a slight positive shift of the asymmetry. However, the effect is not statistically significant.

In order to study the influence of the jet selection we vary the lower cut-off scale of the transverse energy $E_{T}^{c u t}$ and the upper cut-off scale of the $\eta$-range for the clustered objects and apply only those modified jet requirements, but no further selection cuts. The minimal transverse energy scale is gradually increased in 5 $\mathrm{GeV}$ steps from $E_{T}=10 \mathrm{GeV}$ to $E_{T}=20 \mathrm{GeV}$, the maximal permitted $\eta$-range is reduced in 0.5 steps from $\eta_{\text {jet }}^{\text {cut }}=3.0$ to $\eta_{\text {jet }}^{\text {cut }}=1.0$. In the previous study the jet clustering was performed by the $K_{T}$ algorithm at hadron level and the cut-off scale decided how the jets are clustered. In contrast to it, we now have jets that are already clustered by the JETCLU algorithm, and we can now select a certain sample containing events whose jets fulfill particular requirements. Thus, a variation of $E_{T}^{c u t}$ and $\eta_{\text {jet }}^{\text {cut }}$ changes the choice of events and we obtain samples with different event contents. The resulting asymmetry values are presented in table 5.6 in the lower three sections. The actual event selection used in this analysis requires events with jets of a minimal transverse energy of $E_{T} \geq 15 \mathrm{GeV}$ and a maximal pseudorapidity of $|\eta| \leq 2.0$. The corresponding asymmetry value is $A=(2.2 \pm 0.35) \%$ which is consistent with the observed asymmetry $A=(2.6 \pm 0.87) \%$ after the full event selection. The remaining difference is due to the slight influence of the chargedlepton cuts and all the additional selection criteria [35] that are not considered in this study, but the deviation is insignificant within the statistical errors. For this reason we conclude that the shift of the asymmetry $A$ of about 3 percentage points is 


\begin{tabular}{|c|c|c|c|}
\hline & $\begin{array}{c}\text { РҮтніА } \\
A\left(\Delta y \cdot Q_{l}\right)[\%]\end{array}$ & $\begin{array}{c}\text { HERWIG } \\
A\left(\Delta y \cdot Q_{l}\right)[\%]\end{array}$ & $\begin{array}{c}\mathrm{MC} @ \mathrm{NLO} \\
A\left(\Delta y \cdot Q_{l}\right)[\%]\end{array}$ \\
\hline no cuts & $-0.1 \pm 0.27$ & $0.1 \pm 0.39$ & $4.8 \pm 0.27$ \\
\hline full selection & $-3.1 \pm 0.91$ & $-2.7 \pm 1.28$ & $2.6 \pm 0.87$ \\
\hline only CEM cuts & $1.6 \pm 1.0$ & $0.11 \pm 1.40$ & $5.4 \pm 0.94$ \\
\hline only CMUP/CMX cuts & $1.4 \pm 1.0$ & $0.9 \pm 1.5$ & $5.0 \pm 1.03$ \\
\hline$E_{T}^{\text {jet }} \geq 10 \mathrm{GeV},\left|\eta^{\text {jet }}\right| \leq 1.0$ & $-7.3 \pm 0.45$ & $-5.6 \pm 0.64$ & $-0.9 \pm 0.45$ \\
\hline$E_{T}^{j e t} \geq 10 \mathrm{GeV},\left|\eta^{j e t}\right| \leq 1.5$ & $-4.3 \pm 0.34$ & $-3.2 \pm 0.49$ & $2.0 \pm 0.34$ \\
\hline$E_{T}^{j e t} \geq 10 \mathrm{GeV},\left|\eta^{j e t}\right| \leq 2.0$ & $-2.7 \pm 0.31$ & $-1.9 \pm 0.46$ & $3.3 \pm 0.31$ \\
\hline$E_{T}^{j e t} \geq 10 \mathrm{GeV},\left|\eta^{j e t}\right| \leq 2.5$ & $-2.1 \pm 0.30$ & $-1.4 \pm 0.43$ & $3.7 \pm 0.30$ \\
\hline$E_{T}^{j e t} \geq 10 \mathrm{GeV},\left|\eta^{j e t}\right| \leq 3.0$ & $-1.9 \pm 0.30$ & $-1.3 \pm 0.43$ & $3.8 \pm 0.30$ \\
\hline$E_{T}^{\text {jet }} \geq 15 \mathrm{GeV},\left|\eta^{\text {jet }}\right| \leq 1.0$ & $-7.2 \pm 0.53$ & $-6.0 \pm 0.75$ & $-1.4 \pm 0.53$ \\
\hline$E_{T}^{j e t} \geq 15 \mathrm{GeV},\left|\eta^{j e t}\right| \leq 1.5$ & $-4.9 \pm 0.39$ & $-4.0 \pm 0.55$ & $0.9 \pm 0.38$ \\
\hline$E_{T}^{j e t} \geq 15 \mathrm{GeV},\left|\eta^{j e t}\right| \leq 2.0$ & $-3.7 \pm 0.35$ & $-3.2 \pm 0.50$ & $2.2 \pm 0.35$ \\
\hline$E_{T}^{j e t} \geq 15 \mathrm{GeV},\left|\eta^{j e t}\right| \leq 2.5$ & $-3.2 \pm 0.34$ & $-2.7 \pm 0.48$ & $2.6 \pm 0.33$ \\
\hline$E_{T}^{j e t} \geq 15 \mathrm{GeV},\left|\eta^{j e t}\right| \leq 3.0$ & $-3.2 \pm 0.34$ & $-2.6 \pm 0.48$ & $2.7 \pm 0.33$ \\
\hline$E_{T}^{\text {jet }} \geq 20 \mathrm{GeV},\left|\eta^{\text {jet }}\right| \leq 1.0$ & $-8.0 \pm 0.64$ & $-5.7 \pm 0.90$ & $-1.2 \pm 0.63$ \\
\hline$E_{T}^{j e t} \geq 20 \mathrm{GeV},\left|\eta^{j e t}\right| \leq 1.5$ & $-5.9 \pm 0.47$ & $-4.3 \pm 0.65$ & $0.3 \pm 0.46$ \\
\hline$E_{T}^{j e t} \geq 20 \mathrm{GeV},\left|\eta^{j e t}\right| \leq 2.0$ & $-4.9 \pm 0.43$ & $-3.8 \pm 0.59$ & $1.1 \pm 0.41$ \\
\hline$E_{T}^{j e t} \geq 20 \mathrm{GeV},\left|\eta^{j e t}\right| \leq 2.5$ & $-4.6 \pm 0.42$ & $-3.5 \pm 0.57$ & $1.6 \pm 0.40$ \\
\hline$E_{T}^{j e t} \geq 20 \mathrm{GeV},\left|\eta^{j e t}\right| \leq 3.0$ & $-4.6 \pm 0.42$ & $-3.5 \pm 0.57$ & $1.6 \pm 0.40$ \\
\hline
\end{tabular}

Table 5.6: Overview of the resulting asymmetry values $A\left(\Delta y \cdot Q_{l}\right)$ if different groups of selection cuts are applied to the three Monte Carlo event samples. In the first line ("no cuts") one finds the values if no selection cuts are made on the samples; "full selection" presents the values for a full lepton + jets event selection. The following two lines contain the asymmetry values if only cuts concerning CEM electrons are applied, or if only CMUP/CMX muons have to fulfill certain requirements. The lower three sections list the values if only jet cuts are applied.

mainly due to restricting the jet kinematics. The dependence of the asymmetry on the jet definition is visualized in figure 5.7. From this jet selection study we derive mainly two important conclusions. The negative shift of the asymmetry $A\left(\Delta y \cdot Q_{l}\right)$ increases if

- we require jets with higher transverse energies. (The same effect is seen in section 5.1.2 when changing the cut-off $d_{\min }^{\text {cut }}$ of the $K_{T}$ algorithm.)

- we require jets that are produced in a more central detector region.

The shape of the dependence of the asymmetry on the upper $\eta$-range cut-off (figure 5.7) is similar for each used Monte Carlo event generator. For the next-to-leading order generator MC@NLO the distribution (c) is merely shifted upwards about the expected inclusive charge asymmetry $A \approx 5 \%$ compared to the leading order distributions. 


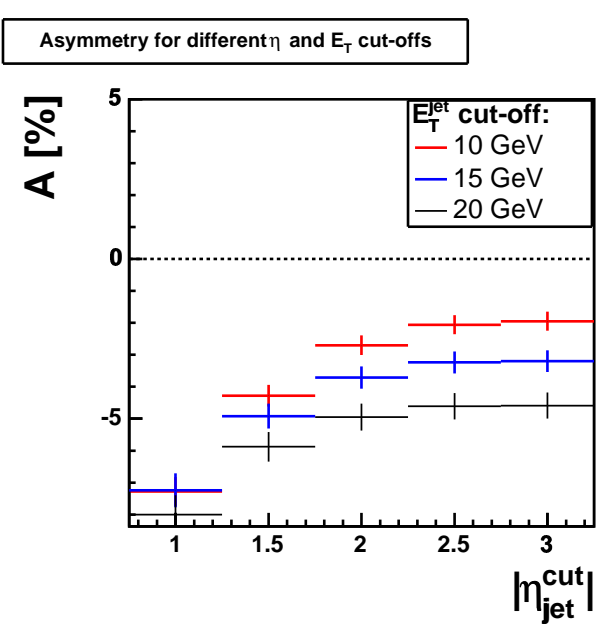

(a) Pythia

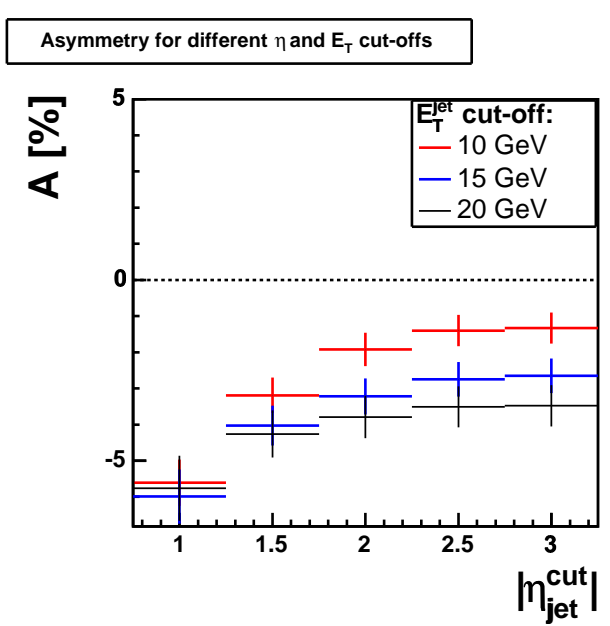

(b) Herwig

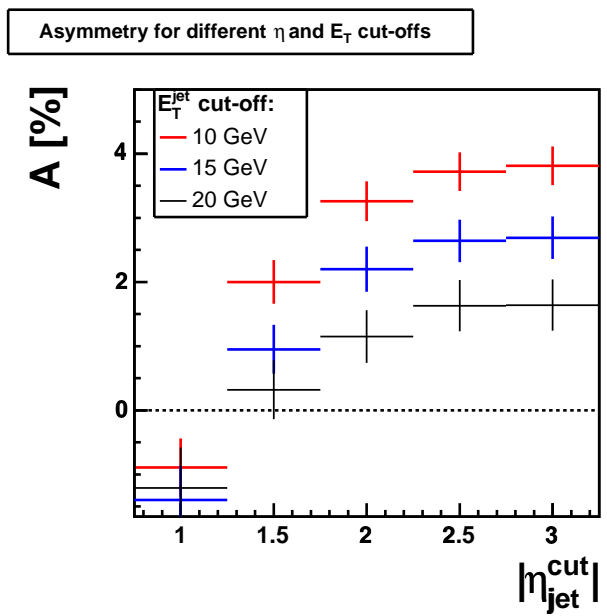

(c) $\mathrm{MC} @ N L O$

Figure 5.7: Visualization of the lower three sections of table 5.6. The dependence of the asymmetry $A$ on the upper $\eta$-range cut-off scale is shown for the three used Monte Carlo samples: PythiA (a), HERwig (b), and MC@NLO (c). The lower cut-off scale of the minimal transverse jet energy $E_{T}^{\text {cut }}$ is varied from $10 \mathrm{GeV}$ to $20 \mathrm{GeV}$. 


\subsection{Effect of Gluon Radiation on Top-Quark Ra- pidity Distributions}

The previous study of the influence of the event selection on the inclusive asymmetry showed, that restricting the jet kinematics leads to a substantial shift of $A$ into the negative direction. Considering the exclusive asymmetry in the different jet bins, we observe a strong dependence. This gives reason to further investigation of the effect of gluon radiation on the inclusive asymmetry. First, we use a PYTHIA Monte Carlo generator to study the influence of different generation processes and the correlation between top-quark rapidity distributions and gluon radiation. Additionally, we use the matrix-element generator MADEVENT and conduct a similar correlation study for the process $q \bar{q} \rightarrow t \bar{t}+j$.

\subsubsection{Asymmetry $A\left(\Delta y \cdot Q_{l}\right)$ for Different $t \bar{t}$ Production Pro- cesses}

To study, whether the production process has an impact on the observed dependence of the asymmetry on the number of jets, we use the PyтHiA Monte Carlo event sample and characterize the events by their production processes. We distinguish between $t \bar{t}$ pair production via gluon fusion and via quark-antiquark annihilation. The corresponding Feynman diagrams are presented in chapter 1.2. The jet clustering at hadron level is done using the $K_{T}$ algorithm within a jet-cone radius set to $\Delta R \leq 0.4$ and a lower cut-off $d_{m i n}^{\text {cut }}$ of 0.0065 . Thus, the events are sorted according to their production process and to the number of observed jets. In figure 5.8 the asymmetry as a function of $N_{\text {jets }}$ is presented for both subsamples. Plot (a) contains

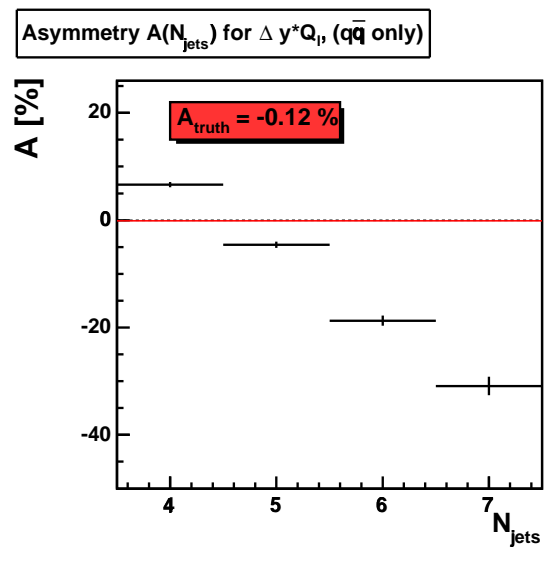

(a)

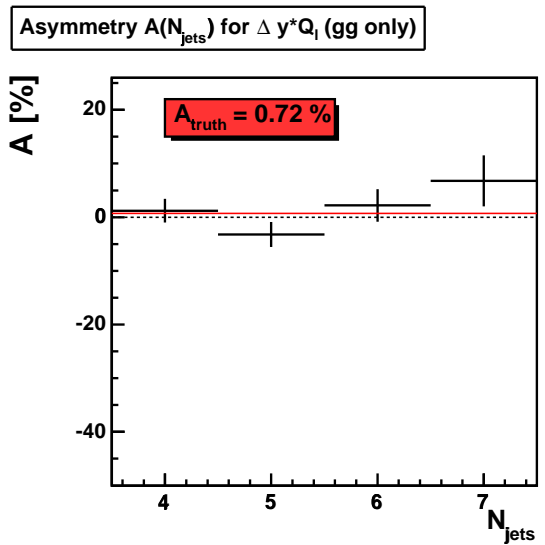

(b)

Figure 5.8: The asymmetry as a function of the number of jets for the PythiA Monte Carlo sample: the linear dependence is only observable for quark-antiquark annihilation (a) whereas $t \bar{t}$ pairs produced via gluon fusion seem to be independent on $N_{\text {jets }}$ (b). 
only events, where the top-quark pair is produced via quark-antiquark annihilation. In this case, the exclusive asymmetry shows the already observed jet bin dependence. On the right hand side (b), the exclusive asymmetry in the gluon-fusion sample is shown for the different jet bins. Unlike the behavior of the exclusive asymmetry in the $q \bar{q}$-annihilation sample, the values of $A$ remain independent of the number of jets in gluon fusion events. The effect that causes the observed linear dependence occurs only if we consider exclusively $t \bar{t}$ pairs that are produced via quark-antiquark annihilation, whereas events with gluon fusion seem to be unaffected. This result is confirmed by the other two Monte Carlo samples, thus we only present the plots for the Pythia Monte Carlo sample.

\subsubsection{Study of the Event Generation with Pythia}

From the studies in the last section we concluded that there is a strong dependence of the measured asymmetry $A$ on the number of observed jets. Additional jets in $t \bar{t}$ events are mostly due to hard gluon radiations off color charged partons like radiation off the incoming partons, or the produced top quarks. We study the influence of initial state gluon radiation (ISR) and final state gluon radiation (FSR) on the charge asymmetry by switching on and off the modeling of these processes in the parton shower Monte Carlo. In order to speedily produce event samples with different settings a stand-alone version of PутніA is used. In addition, the influence of modeling multiple interactions is investigated.

We analyze the influence on the distributions of three different variables: the rapidities of the top and the antitop quark, $y_{t}$ and $y_{\bar{t}}$, and the rapidity difference $\Delta y \cdot Q_{l}$. The asymmetry of these parameters is analogically defined as:

$$
A=\frac{N_{e v t s}(X>0)-N_{e v t s}(X<0)}{N_{e v t s}(X>0)+N_{e v t s}(X<0)},
$$

where $\mathrm{X}$ denotes $y_{t}, y_{\bar{t}}$ and $\Delta y \cdot Q_{l}$ respectively.

In figure 5.9 the asymmetry values of the three distributions $\left(y_{t}, y_{\bar{t}}\right.$ and $\left.\Delta y \cdot Q_{l}\right)$ as a function of $N_{\text {jets }}$ in case of a full event generation are compared to those if ISR (MSTP switch 61) and FSR (MSTP switch 71) are switched off respectively. For each process configuration a sample of 200,000 events was produced. No selection cuts except for the classification of the jets by a $K_{T}$ algorithm $\left(d_{\min }^{\text {cut }}=0.0065\right.$, $\Delta R \leq 0.4)$ are applied to the samples. Since we have seen that the dependence of the asymmetry on $N_{\text {jets }}$ occurs only in the distributions of events whose $t \bar{t}$ pairs are produced via quark-antiquark annihilation, we hence consider only such events. The plot on the upper right shows the distribution of the exclusive asymmetry for the rapidity $y_{t}$ of the top quark. In the four-jets bin, the top quarks are produced rather in the proton direction. The value of $A$ is positive. If one additional jet is produced in the event, the value of $A$ gets negative, i.e. the produced top quarks are rather produced in the direction of the antiproton. The same effect is visible concerning the rapidity of the antitop quark. One has to consider that the $\mathrm{z}$ component of the incoming antiquark is negative due to the oppositional heading of the antiproton, 
but the definition of the asymmetry remains equal. Thus, the asymmetry of the antitop-quark rapidity $y_{\bar{t}}$ behaves reversed compared to the asymmetry of the topquark rapidity $y_{t}$.

If ISR is turned off, the asymmetry remains constant for $N_{\text {jets }}=4$ and $N_{\text {jets }}=5$; if ISR and FSR are both switched off simultaneously, shown in figure 5.10, this even holds for $N_{\text {jets }}=6$. Due to this observation we conclude that the dependence of the asymmetry on the number of jets is associated with the emergence of initial and final state gluon radiation.

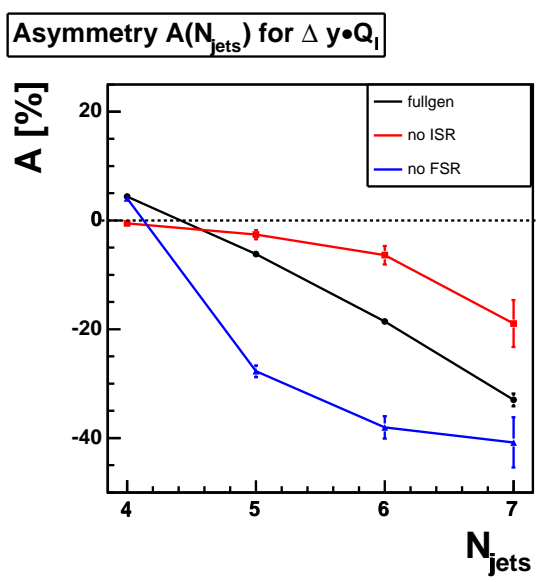

(a)

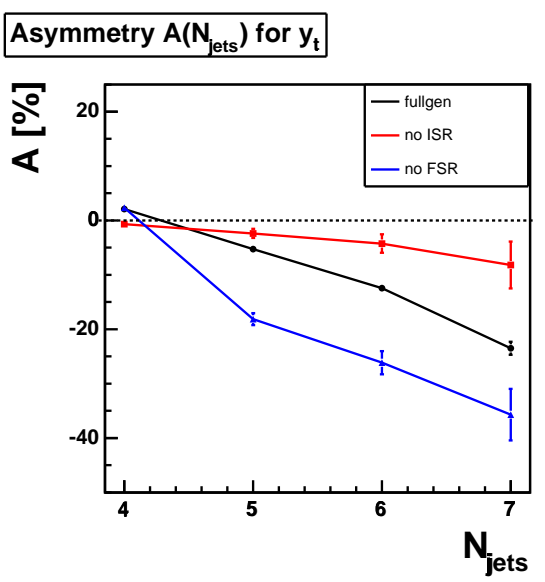

(b)

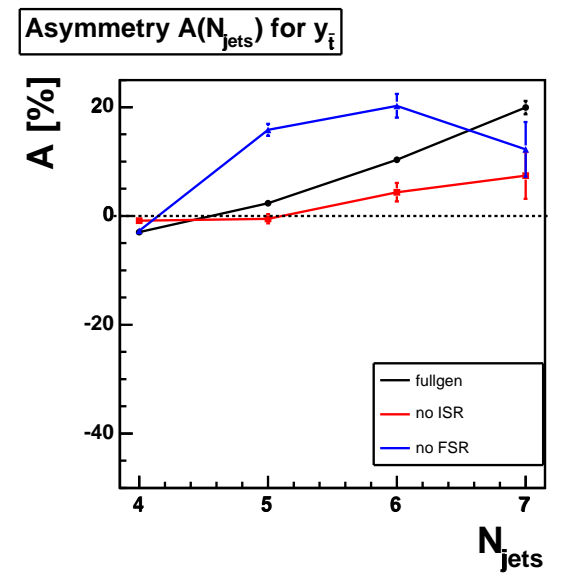

(c)

Figure 5.9: The asymmetry as a function of $N_{\text {jets }}$ for $\Delta y \cdot Q_{l}$ (a), for $y_{t}(\mathrm{~b})$ and for $y_{\bar{t}}(\mathrm{c})$. Different processes are switched off for the event generation. The red line represents the linear behavior in the case of a full event generation with PyтнiA, the black line shows the trend if final state radiation is switched off, the blue line gives the distribution if initial state gluon radiation is turned off. No selection cuts except for the jet clustering are applied. 


\section{2. $\quad$ Effect of Gluon Radiation on Top-Quark Rapidity Distributions 63}

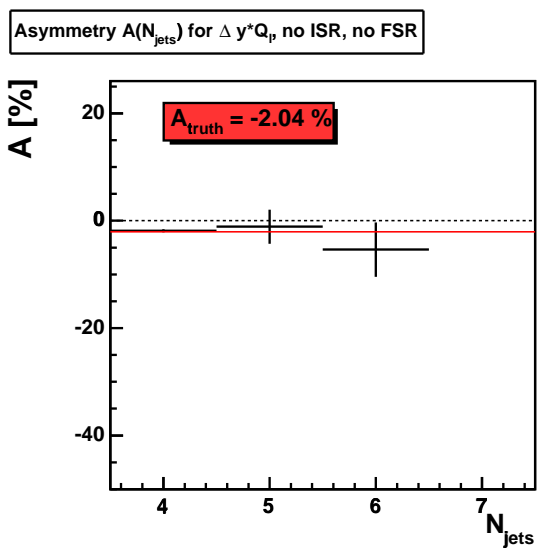

(a)

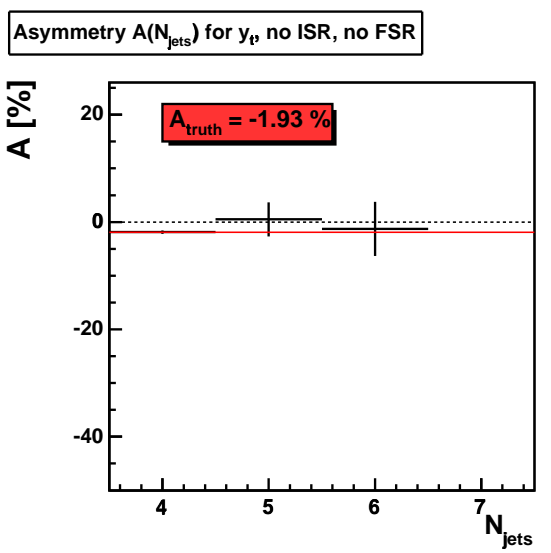

(b)

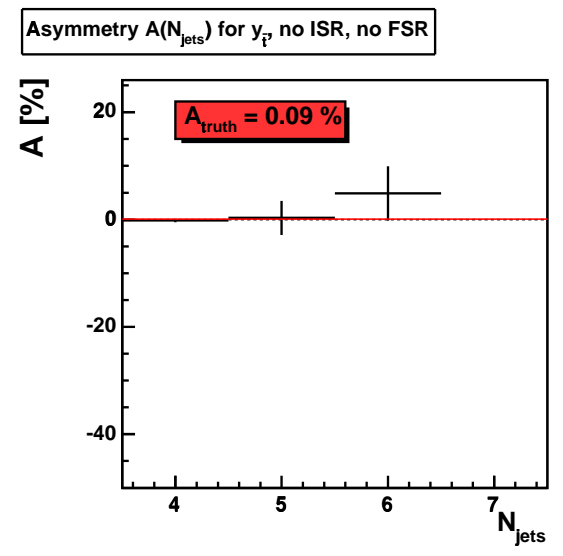

(c)

Figure 5.10: The asymmetry as a function of $N_{\text {jets }}$ for $\Delta y \cdot Q_{l}$ (a), for $y_{t}$ (b) and for $y_{\bar{t}}$ (c), ISR and FSR are switched off simultaneously. The red line represents the value of the asymmetry for the Pythia Monte Carlo truth distribution, i.e. no jet requirement is made; the same value is displayed in the red colored box. No selection cuts except for the jet clustering are applied.

If multiple interactions are turned off (MSTP switch 81), the exclusive asymmetry remains unchanged. The linear trend of $A$ occurs for each of the three variables, see figure 5.11. We conclude that multiple interactions do not influence the rapidity distributions of the produced top and antitop quarks.

Recapitulating the previous studies we can summarize the following findings:

- We observe a linear dependence of the asymmetry $A$ for the variables $\Delta y \cdot Q_{l}$, $y_{t}$ and $y_{\bar{t}}$ on the number of jets $N_{\text {jets }}$ on Monte Carlo level.

- The effect occurs exclusively in events in which the $t \bar{t}$ pairs are produced via quark-antiquark annihilation. 


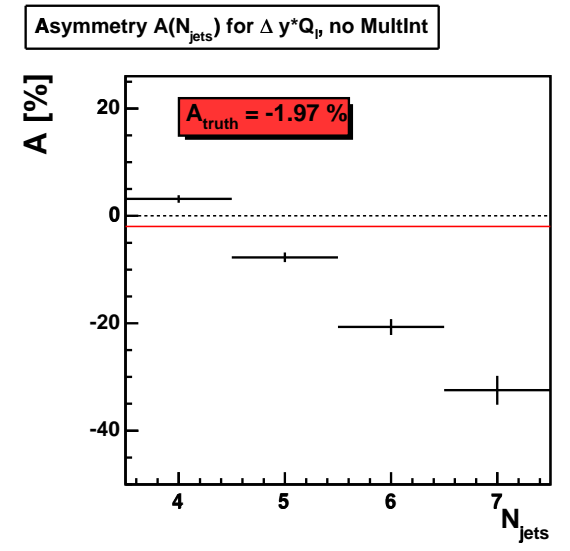

(a)

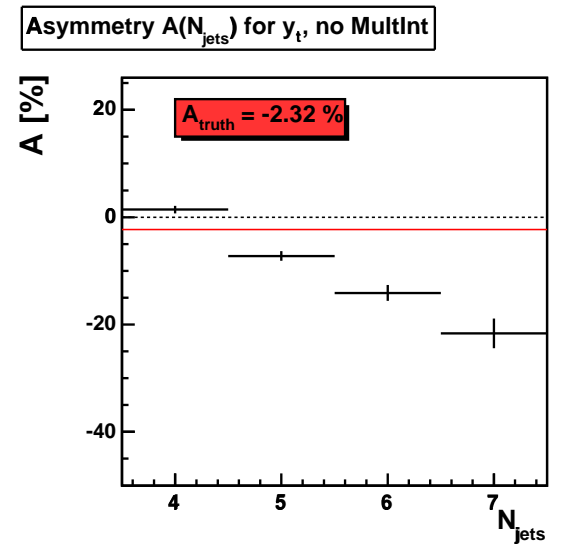

(b)

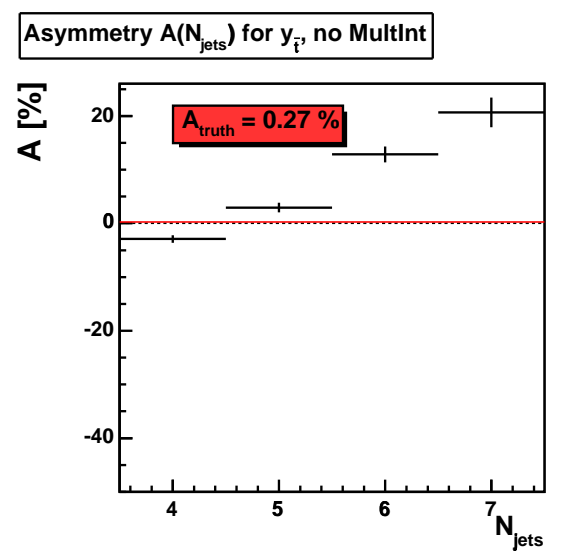

(c)

Figure 5.11: The asymmetry as a function of $N_{\text {jets }}$ for $\Delta y \cdot Q_{l}$ (a), for $y_{t}$ (b) and for $y_{\bar{t}}$ (c) if multiple interactions are switched off in the PyтHIA event generation. No selection cuts except for the jet clustering are applied.

- The rapidity distributions of the top and the antitop quark are strongly affected by gluon radiation.

An effect on the asymmetry, which is calculated in the rapidity difference of the top and the antitop quark, implies that the reason of the effect has to be sensitive to the difference between particle and antiparticle. The fact, that we observe this effect only in events, where the top-quark pair is produced via quark-antiquark annihilation, combined with the finding that gluon radiation plays a role, raises the question, how the incoming quark and the produced top quark (incoming antiquark and produced antitop quark) are connected. We assume that the color flow which 


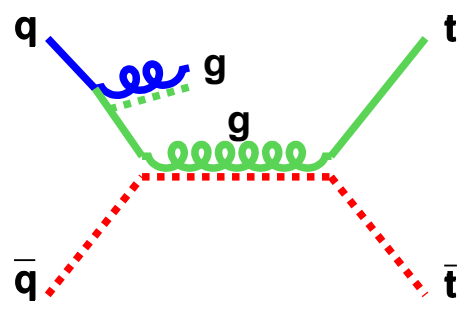

(a)

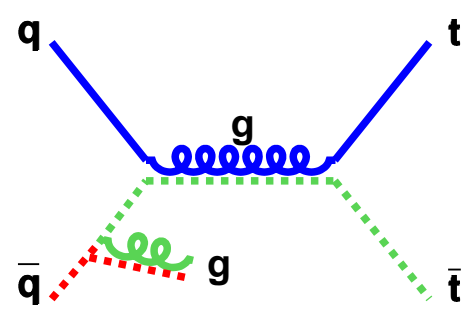

(b)

Figure 5.12: Illustration of the color flow in a $t \bar{t}$ event produced via quark-antiquark annihilation. Graph (a) shows the case that a gluon is radiated off the incoming quark, in (b) the gluon is radiated off the antiquark. Color charges are represented by the solid colored lines, anticolor charges by the dotted lines. Although the color charge of the produced top (antitop) quark is changed by gluon radiation, the connection to the initial quark (antiquark) remains due to the fact that quarks always carry a color charge, whereas antiquarks can only be anticolor-charged.

is a conserved quantity in the hard process may constitute such a connection. If we consider the color flow in top pair production via quark-antiquark annihilation, the incoming quark carries a color charge $c_{q}$ and the antiquark an anticolor charge $\bar{c}_{\bar{q}}$. When they annihilate, a virtual gluon with the color charge $c_{q} \bar{c}_{\bar{q}}$ passes them to the produced top and antitop quark, whereby the top must take over the color charge $c_{q}$. Thus, the color flow at the parton $(q-t)$ and the antiparton $(\bar{q}-\bar{t})$ side can uniquely be associated with the original proton or antiproton. This association remains if a gluon is radiated off the incoming quark or antiquark. Figure 5.12 illustrates qualitatively the color flow in $q \bar{q} \rightarrow t \bar{t}$ events in the case of initial state gluon radiation off the incoming quark (a) or antiquark (b).

The color charge of the produced top quark indeed changes due to the radiation, but the unambiguous connection of the produced quark with the original proton remains. In the case of $t \bar{t}$ pair production via gluon fusion there is no unique connection between proton and top quark, and antiproton and antitop quark. Since an incoming gluon always carries a color charge and an anticolor charge it is impossible to decide whether it comes from a proton or an antiproton. The produced top quark is not necessarily color connected exclusively to the proton, thus there is no observable effect on the top-quark rapidity distributions.

A closer look to the events generated with the PyтнiA Monte Carlo generator delivers further evidence for that hypothesis. In table 5.7 an extract of an event record including the string information is shown for a typical $t \bar{t}$ pair event produced via quark-antiquark annihilation. After the hard scattering process $(\mathrm{KS}=21)$ the built strings are listed, whereby the label "A" indicates the beginning of the string, "I" the appendant particles and "V" the end of the string. As can be seen in the event record a string is built between the $b$ quark from the top quark and the proton remnant $(I=22 \rightarrow I=28)$ and between the $\bar{b}$ quark from the antitop and the antiproton remnant $(I=29 \rightarrow I=40)$, respectively. 
Chapter 5. MC Study of Asymmetry in Top-Pair Production

\begin{tabular}{|c|c|c|c|c|c|c|c|c|c|}
\hline I & particle,jet & $\mathrm{KS}$ & $\mathrm{KF}$ & orig & $\mathrm{px}$ & py & $\mathrm{pz}$ & $\mathrm{E}$ & $\mathrm{m}$ \\
\hline$\overline{1}$ & $! p+!$ & 21 & 2212 & 0 & 0.000 & 0.000 & 980.000 & 980.000 & 0.938 \\
\hline 2 & !pbar-! & 21 & -2212 & 0 & 0.000 & 0.000 & -980.000 & 980.000 & 0.938 \\
\hline 3 & !u! & 21 & 2 & 1 & 1.330 & 3.665 & 323.559 & 323.582 & 0.000 \\
\hline 4 & !ubar! & 21 & -2 & 2 & -0.992 & 0.905 & -170.679 & 170.684 & 0.000 \\
\hline 5 & !u! & 21 & 2 & 3 & 0.159 & 1.814 & 262.340 & 262.347 & 0.000 \\
\hline 6 & !ubar! & 21 & -2 & 4 & 0.982 & 3.287 & -160.250 & 160.287 & 0.000 \\
\hline 7 & !t! & 21 & 6 & 0 & -22.012 & -36.533 & -49.958 & 186.309 & 174.344 \\
\hline 8 & !tbar! & 21 & -6 & 0 & 23.153 & 41.634 & 152.048 & 236.325 & 174.532 \\
\hline 9 & !W+! & 21 & 24 & 7 & -35.822 & -22.768 & -98.043 & 134.198 & 81.210 \\
\hline 10 & !b! & 21 & 5 & 7 & 13.811 & -13.765 & 48.086 & 52.110 & 4.800 \\
\hline 11 & !W-! & 21 & -24 & 8 & -52.503 & 7.683 & 99.288 & 137.922 & 79.679 \\
\hline 12 & !bbar! & 21 & -5 & 8 & 75.656 & 33.951 & 52.760 & 98.403 & 4.800 \\
\hline 13 & !sbar! & 21 & -3 & 9 & 19.211 & -21.850 & -10.356 & 30.886 & 0.500 \\
\hline 14 & !c! & 21 & 4 & 9 & -54.613 & -0.773 & -86.484 & 102.299 & 1.500 \\
\hline 15 & !e-! & 21 & 11 & 11 & -26.254 & -22.238 & 87.912 & 94.405 & 0.001 \\
\hline 16 & !nu_ebar! & 21 & -12 & 11 & -17.790 & 30.916 & 5.090 & 36.030 & 0.000 \\
\hline 17 & $(\mathrm{~W}+)$ & 11 & 24 & 9 & $\begin{array}{l}-35.403 \\
\end{array}$ & $\begin{array}{l}-22.623 \\
\end{array}$ & $\begin{array}{c}-96.840 \\
\end{array}$ & 133.185 & 81.210 \\
\hline 18 & $(\mathrm{~W}-)$ & 11 & -24 & 11 & -44.045 & 8.677 & 93.002 & 130.435 & 79.679 \\
\hline 19 & nu_ebar & 1 & -12 & 16 & -17.790 & 30.916 & 5.090 & 36.030 & 0.000 \\
\hline 20 & e- & 1 & 11 & 15 & -26.254 & -22.238 & 87.910 & 94.403 & 0.001 \\
\hline 21 & gamma & 1 & 22 & 15 & -0.001 & -0.001 & 0.002 & 0.002 & 0.000 \\
\hline 22 & (b) $\mathrm{A}$ & 12 & 5 & 10 & 15.503 & -13.397 & 39.493 & 44.750 & 4.800 \\
\hline 23 & (g) I & 12 & 21 & 10 & 0.161 & 0.721 & 3.265 & 3.348 & 0.000 \\
\hline 24 & (g) I & 12 & 21 & 10 & -1.376 & -1.000 & 1.593 & 2.330 & 0.000 \\
\hline 25 & (g) I & 12 & 21 & 10 & -0.897 & -0.234 & 2.531 & 2.695 & 0.000 \\
\hline 26 & (g) I & 12 & 21 & 3 & 0.534 & 1.862 & 56.090 & 56.123 & 0.000 \\
\hline 27 & (g) I & 12 & 21 & 3 & 0.637 & -0.014 & 4.703 & 4.746 & 0.000 \\
\hline 28 & (ud_0) V & 11 & 2101 & 1 & -1.330 & -3.665 & 656.184 & 656.195 & 0.579 \\
\hline 29 & (bbar) A & 12 & -5 & 12 & 59.044 & 15.721 & 58.293 & 84.584 & 4.800 \\
\hline 30 & (g) I & 12 & 21 & 12 & 3.262 & 1.267 & 1.206 & 3.701 & 0.000 \\
\hline 31 & (g) I & 12 & 21 & 12 & 0.638 & 0.956 & -0.446 & 1.233 & 0.000 \\
\hline 32 & (g) I & 12 & 21 & 12 & 2.781 & 8.901 & 1.778 & 9.494 & 0.000 \\
\hline 33 & (g) I & 12 & 21 & 12 & 0.063 & 1.094 & -0.981 & 1.471 & 0.000 \\
\hline 34 & (g) I & 12 & 21 & 12 & 0.518 & 1.998 & 0.266 & 2.082 & 0.000 \\
\hline 35 & (g) I & 12 & 21 & 12 & 0.891 & 3.020 & -1.070 & 3.325 & 0.000 \\
\hline 36 & (g) I & 12 & 21 & 0 & 1.443 & -0.343 & -2.286 & 2.725 & 0.000 \\
\hline 37 & (g) I & 12 & 21 & 0 & -1.443 & 0.343 & -31.911 & 31.946 & 0.000 \\
\hline 38 & (g) I & 12 & 21 & 4 & -0.313 & -0.936 & -0.716 & 1.220 & 0.000 \\
\hline 39 & (g) I & 12 & 21 & 4 & -1.661 & -1.443 & -9.287 & 9.544 & 0.000 \\
\hline 40 & $(\mathrm{ud}-0 \mathrm{bar}) \mathrm{V}$ & 11 & -2101 & 2 & 0.992 & -0.905 & -774.866 & 774.868 & 0.579 \\
\hline 41 & (sbar) A & 12 & -3 & 13 & 16.375 & -11.922 & -5.577 & 21.015 & 0.500 \\
\hline 42 & (g) I & 12 & 21 & 13 & 1.219 & -0.356 & -0.886 & 1.548 & 0.000 \\
\hline 43 & (g) I & 12 & 21 & 13 & 0.229 & -0.203 & -0.293 & 0.423 & 0.000 \\
\hline 44 & (d) $\mathrm{V}$ & 11 & 1 & 13 & -0.827 & -4.903 & -3.487 & 6.082 & 0.330 \\
\hline 45 & (c) $\mathrm{A}$ & 12 & 4 & 14 & -34.741 & 4.455 & -54.324 & 64.653 & 1.500 \\
\hline 46 & (g) I & 12 & 21 & 14 & -6.310 & 0.253 & -11.143 & 12.808 & 0.000 \\
\hline 47 & (g) I & 12 & 21 & 14 & -2.964 & -0.606 & -4.290 & 5.249 & 0.000 \\
\hline 48 & (g) I & 12 & 21 & 14 & -1.742 & -1.807 & -2.725 & 3.705 & 0.000 \\
\hline 49 & (g) I & 12 & 21 & 14 & -5.486 & -3.955 & -10.388 & 12.395 & 0.000 \\
\hline 50 & (dbar) V & 11 & -1 & 13 & -1.157 & -3.579 & -3.728 & 5.306 & 0.330 \\
\hline
\end{tabular}

Table 5.7: Extract of a Pythia Monte Carlo "Pylist": I is the line number of the particle, KS is the status code, $\mathrm{KF}$ is the identification number (ID); "orig" is the line number of the parent particle $; p_{x}, p_{y}, p_{z}$ are the momenta in $\mathrm{x}, \mathrm{y}, \mathrm{z}$, direction in $[\mathrm{GeV} / \mathrm{c}] ; \mathrm{E}$ is the energy in $[\mathrm{GeV}] ; \mathrm{m}$ is the mass in $\left[\mathrm{GeV} / c^{2}\right]$ 
In these strings gluons are included which were radiated from the incoming quark $(I=26,27)$ (antiquark $(I=38,39))$. Thus the partons, and the antipartons respectively, are color connected objects in the PYTHIA event generation. To support our hypothesis we study the correlation between the strength of the radiation and the shift of the mean of the rapidity of the produced top quark, whereby we only consider events with $t \bar{t}$ pair production via quark-antiquark annihilation. Three different variables are considered to describe the strength of the initial state gluon radiation:

1. The four-vectors of the ISR gluons listed in the corresponding string are summed up to one radiated object. The transverse momentum of this object is given by $p_{T}^{I S R}(q)\left(p_{T}^{I S R}(\bar{q})\right)$.

2. The rapidity of this string-summed-up object is labeled with $y_{I S R}(q)\left(y_{I S R}(\bar{q})\right)$.

3. The four-vectors of the incoming quark (antiquark) before and after the radiation are compared and the difference is taken as the radiated object. The transverse momentum of this object is given by $\Delta p_{T}^{I S R}(q)\left(\Delta p_{T}^{I S R}(\bar{q})\right)$.

The distributions of the transverse momenta of the radiation are shown in figure 5.13. The number of entries on the $y$ axis is plotted logarithmically to show the tails of the distributions.



(a)

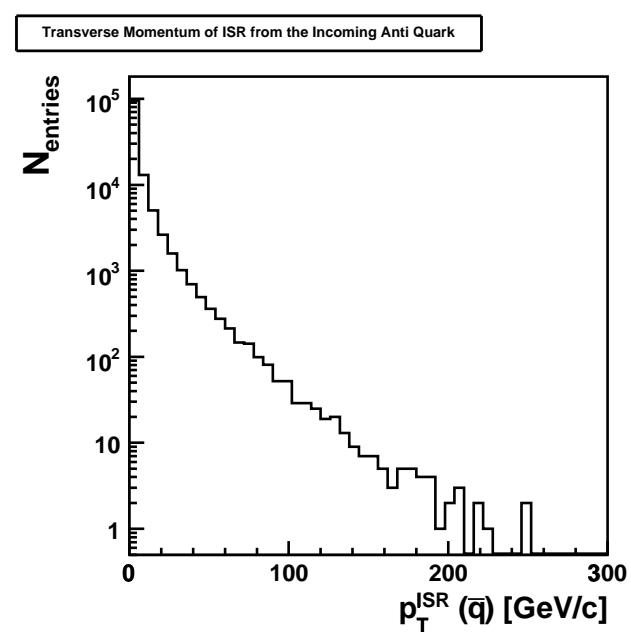

(b)

Figure 5.13: The PyтнiA Monte Carlo distribution of the transverse momentum of the radiation from the incoming quark (a) and the incoming antiquark (b).

To ascertain in which way the rapidity distributions are affected by the strength of the initial state gluon radiation, we plot the rapidity as a function of each of the three radiation variables. Figure 5.14 shows the two dimensional correlation plots 
for the rapidity of the top quark versus the first radiation variable $p_{T}^{I S R}(q)$ (a), and for the antitop quark respectively (c). To improve the visualization of the effect of different radiation strengths on the rapidity distributions, a projection of the slices in $p_{T}$ direction is made. The projections are then fitted with a Gaussian function and the distribution of the corresponding means $\mu$ is plotted as a function of the radiation variable $(b, d)$. We have already seen that in the four-jets bin, i.e. if the jets emerge quasi exclusively from the partons of the decaying top and antitop quark, the value of the asymmetry is a bit above zero. If initial state gluon radiation takes place and additional jets are observed, the mean of the $y_{t}$ distribution is shifted into the negative direction. The same effect can be observed here: in the first bin of the distribution of the means, representing a rather soft radiation, the mean is slightly above zero. As soon as the radiation reaches a certain strength, the mean of the rapidity distribution becomes negative. The description of the strength of the radiation by $\Delta p_{T}^{I S R}(q)\left(\Delta p_{T}^{I S R}(\bar{q})\right)$ delivers similar results except for the first bin, see figure 5.15 (a-d). If the radiation is negligible, the mean of the rapidity distribution is very close to zero. If the rapidity is considered to describe the radiation, it leads to a consistent result, see figure 5.16 (a-d). For a high rapidity, i.e. the radiation points quasi along the proton direction (along the antiproton direction) the rapidity distribution of the top quark (antitop quark) is shifted by a constant factor into the positive (negative) region. Once the rapidity of the radiation falls below a value of $y_{I S R}(q) \approx 3$ (rises above $y_{I S R}(\bar{q}) \approx-3$ ), the rapidity of the top quark (antitop quark) seems to be influenced in the already observed way. 


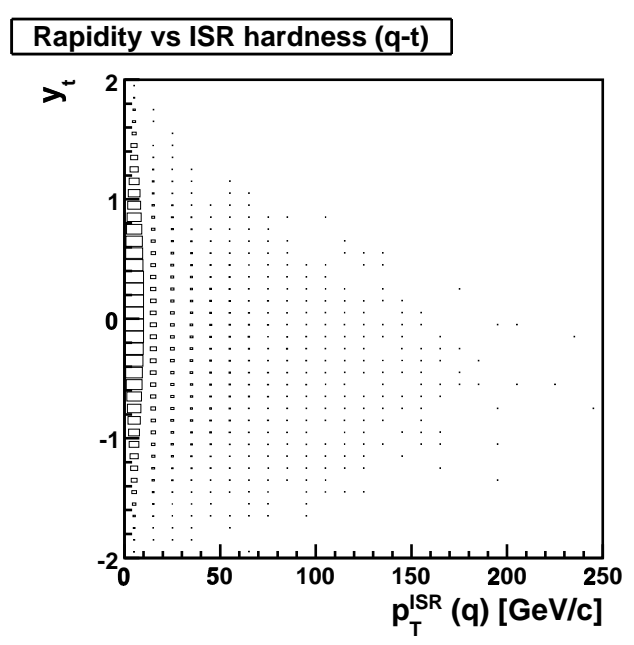

(a)

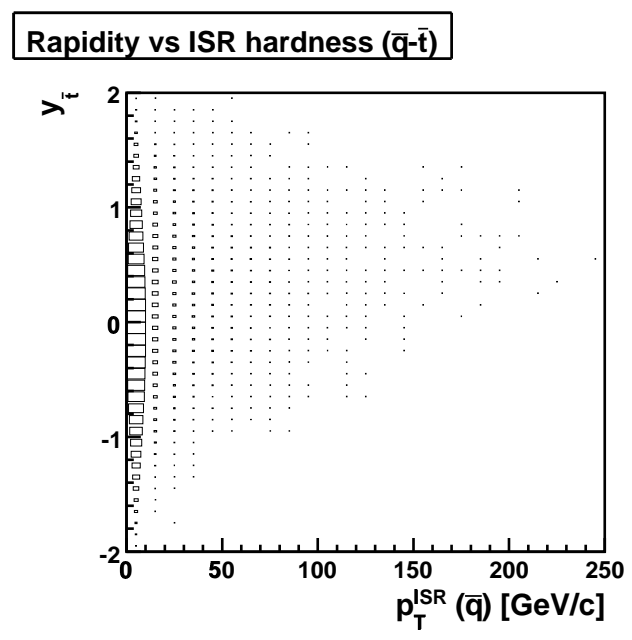

(c)

\section{Mean $\mu$ of Fitted Y Slices $\left(y_{\mathbf{t}}\right)$}

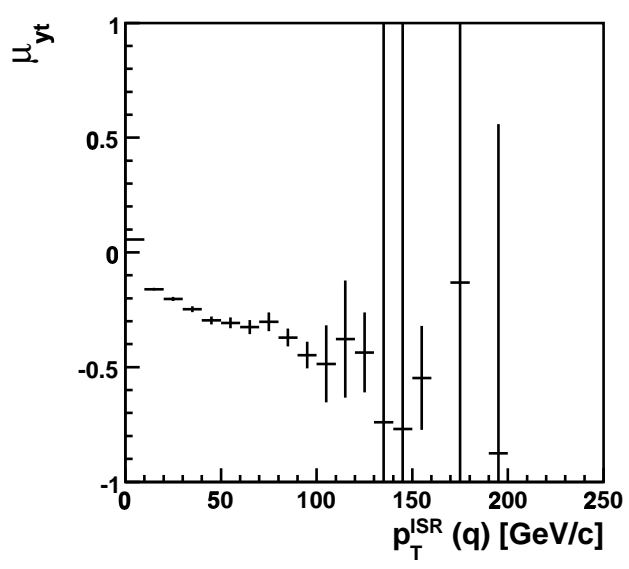

(b)

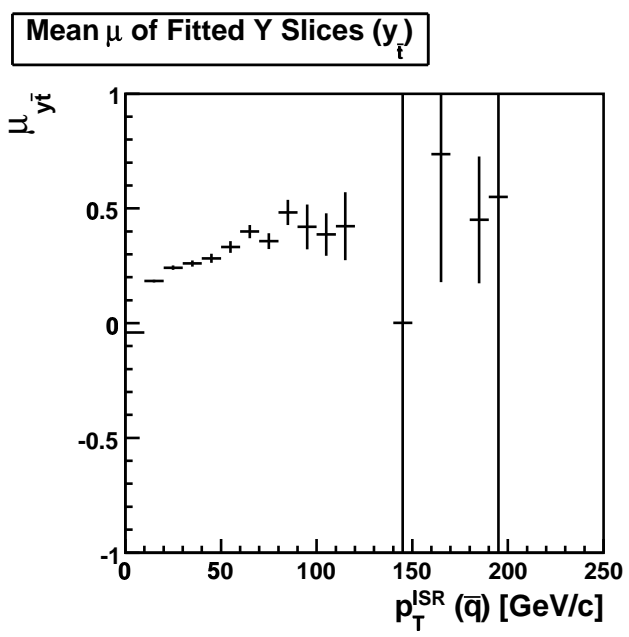

(d)

Figure 5.14: The correlation plots (a, c) and the distributions of the means of the fitted y slices (b, d) for the top quark (a, b) and the antitop quarks (c, d); the strength of the radiation is described by the transverse momentum of the cumulative four-vector of all ISR gluons in the string, $p_{T}^{I S R}(q)$ $\left(p_{T}^{I S R}(\bar{q})\right)$. 


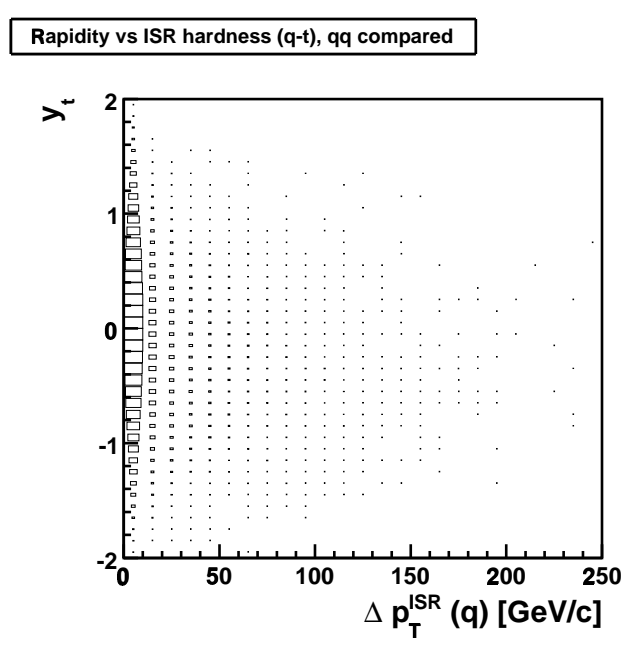

(a)

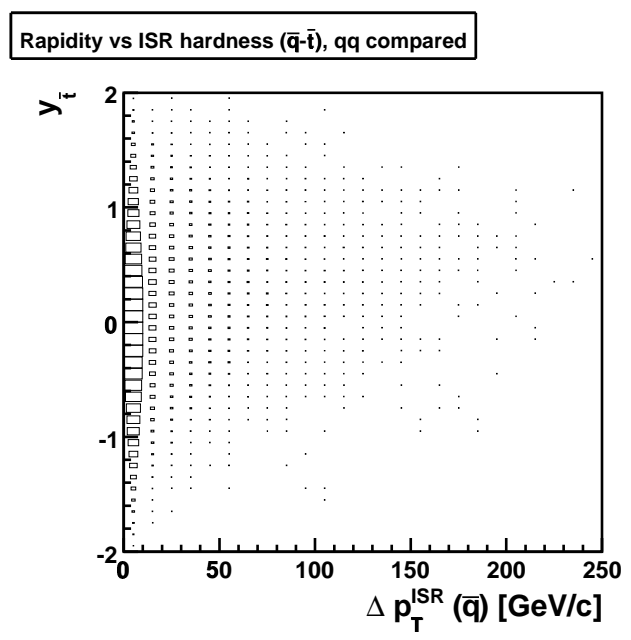

(c)

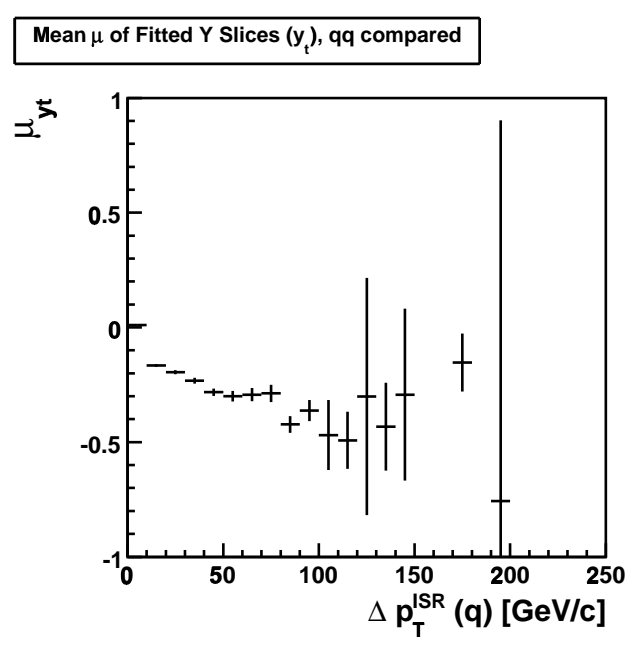

(b)

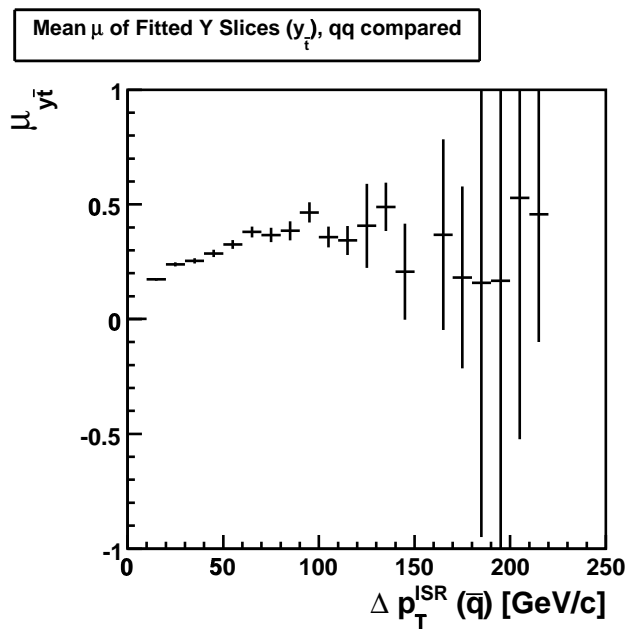

(d)

Figure 5.15: The correlation plots (a, c) and the distributions of the means of the fitted y slices (b, d) for the top quark $(a, b)$ and the antitop quarks $(c, d)$; the strength of the radiation is described by the transverse momentum of the difference between the four-vectors of an incoming parton before and after the radiation, $\Delta p_{T}^{I S R}(q)\left(\Delta p_{T}^{I S R}(\bar{q})\right)$. 


\section{Rapidity t vs Rapidity q}

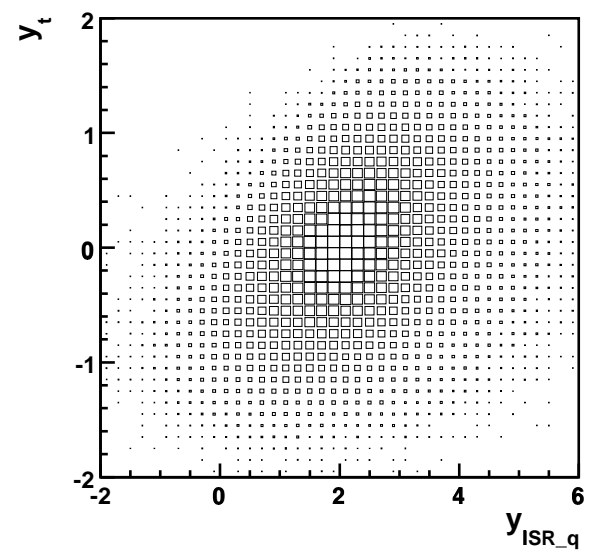

(a)

\section{Rapidity t vs Rapidity $\overline{\mathbf{q}}$}

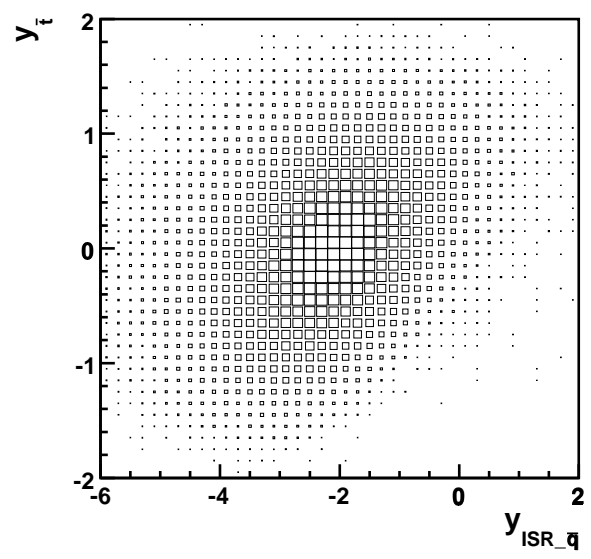

(c)

\section{Mean $\mu$ of Fitted Y Slices $\left(y_{t}\right)$}

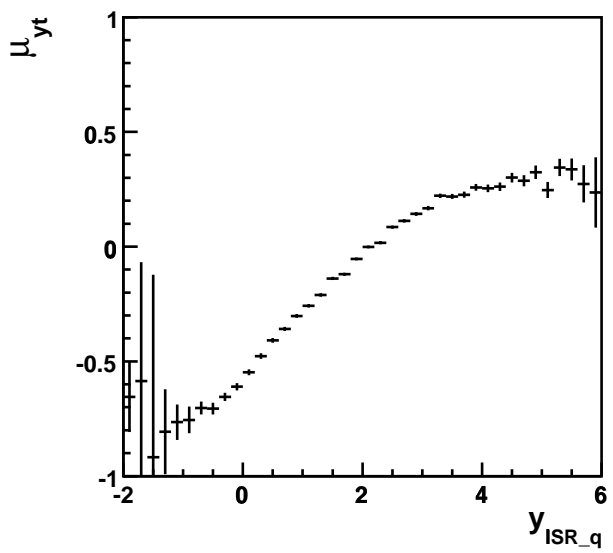

(b)
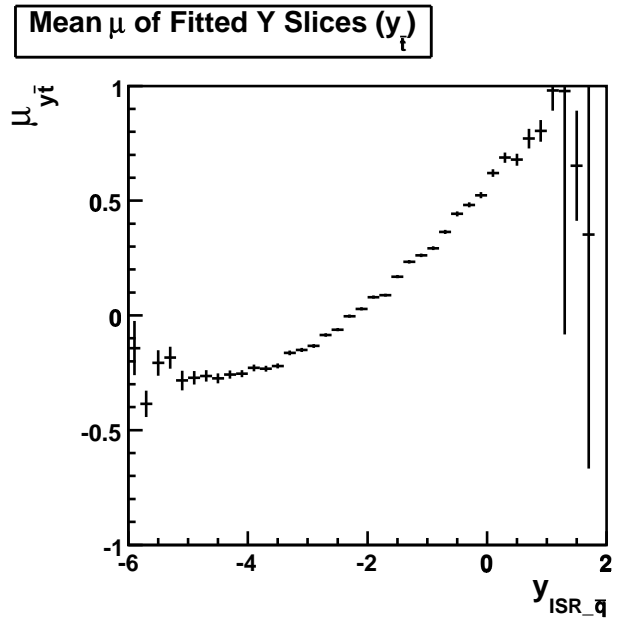

(d)

Figure 5.16: The correlation plots (a, c) and the distributions of the means of the fitted y slices (b, d) for the top quark (a, b) and the antitop quarks (c, d); the strength of the radiation is described by the rapidity of the cumulative four-vector of all ISR gluons in the string, $y_{I S R}(q)\left(y_{I S R}(\bar{q})\right)$. 


\subsubsection{Study of the Event Generation with MadEvent}

So far, the Monte Carlo studies were based on event generators including parton showering (LO: PYThiA, HERwig; NLO: MC@NLO). To check the influence of the parton shower modeling on the observed asymmetry dependence, the matrix-element event generator MADEvENT is used. We generate the process $p \bar{p} \rightarrow t \bar{t} j$, i.e. $t \bar{t}$ events where one additional gluon or $q \bar{q}$-pair labeled with "j" is radiated. If hard gluon radiation at matrix-element level plays a role in top pair production, the rapidity distributions of the top quarks in this process should be shifted. Since the generated process corresponds to the event topology in the five-jets bin, we expect a negative shift of the rapidity distribution of top quarks, and a positive mean of the antitop quark one.

Similar as in section 5.2.2 we consider only the dominant production mechanism, quark-antiquark annihilation, because no asymmetry was found in case of gluon fusion. Table 5.8 shows a typical printout of the MADEvent generator. The particles occurring in the process $p \bar{p} \rightarrow t \bar{t} j$ are: two incoming quarks (particle 1 and particle 2 ), the radiated hard gluon (particle 5) and the produced top and antitop quark (particle 3 and particle 4). The color attribute of the particles is encoded in the lines ICol1 and ICol2, whereby the anticolors are always listed in ICol1 and the colors in ICol2. The numbers 501, 502 and 503 represent the three different color charges.

\begin{tabular}{c||c|c|c|c|c} 
& particle 1 & particle 2 & particle 3 & particle 4 & particle 5 \\
\hline \hline ID & 2 & -2 & 6 & -6 & 21 \\
\hline ICol1 & 0 & 503 & 0 & 502 & 503 \\
ICol2 & 501 & 0 & 501 & 0 & 502 \\
\hline $\mathrm{E}$ & $0.20607 \mathrm{E}+03$ & $0.27394 \mathrm{E}+03$ & $0.23456 \mathrm{E}+03$ & $0.20617 \mathrm{E}+03$ & $0.39281 \mathrm{E}+02$ \\
$\mathrm{Px}$ & $0.00000 \mathrm{E}+00$ & $0.00000 \mathrm{E}+00$ & $-0.1144 \mathrm{E}+03$ & $0.100432 \mathrm{E}+03$ & $0.10043 \mathrm{E}+03$ \\
$\mathrm{Py}$ & $0.00000 \mathrm{E}+00$ & $0.00000 \mathrm{E}+00$ & $0.72595 \mathrm{E}+02$ & $-0.4375 \mathrm{E}+02$ & $-0.28843 \mathrm{E}+02$ \\
$\mathrm{Pz}$ & $0.20607 \mathrm{E}+03$ & $-0.2739 \mathrm{E}+03$ & $-0.79248 \mathrm{E}+02$ & $-0.11333 \mathrm{E}+02$ & $0.22714 \mathrm{E}+02$ \\
\hline \hline
\end{tabular}

Table 5.8: A MADEvent event record: ID is the particle identity, ICol1 the anticolor charge, ICol2 the color charge, $\mathrm{E}$ the energy in $\mathrm{GeV}, \mathrm{Px}, \mathrm{Py}$ and $\mathrm{Pz}$ the transverse momentum components in $\mathrm{GeV} / c$ for each of the five particles.

Several Feynman diagrams contribute to the process $q \bar{q} \rightarrow t \bar{t} j$, including radiation in the initial as well as in the final state. Since MADEVEnT generates inclusive event rates, there is no possibility to differentiate between pure ISR and FSR events. But, to investigate the radiation effects observed in section 5.2.2, it is sufficient to trace back whether the object was radiated off the quark side or off the antiquark side. This is done on the basis of the color flow information encoded in ICOL1 and ICOL2. As can be seen from the example event in table 5.8 the color flow is conserved regardless whether the event was ISR or FSR. In the case of ISR the incoming antiquark with anticolor 503 radiates a gluon that carries anti-503 and 502, and thus changes to an anti-502 colored particle. This anti-502 is then passed on to the produced antitop quark. The produced top quark receives its color of the type 501 from the incoming quark. In the case this event has been a FSR event the two 


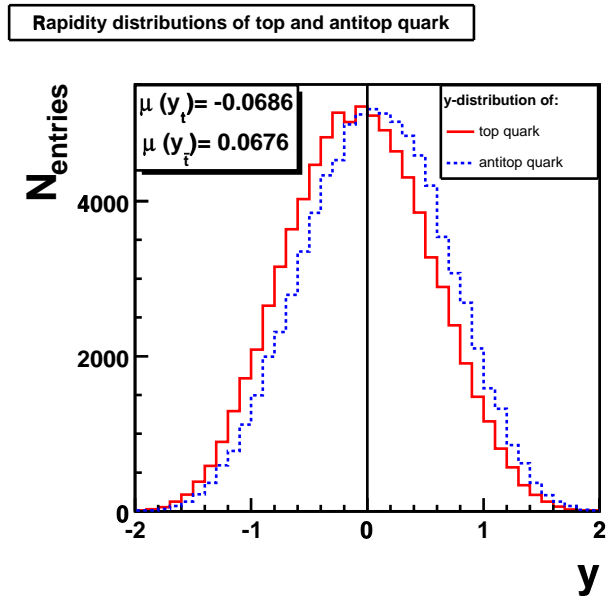

Figure 5.17: The distributions of the rapidities of the top quark and of the antitop quark, the events are generated with MADEvENT.

incoming quarks build an intermediate gluon with color 501 and anti-503, the 501 is then passed to the top quark as before while the anti-503 is passed to the antitop. The antitop then radiates a gluon with anti-503 and 502 leaving an antitop with anti-502.

In the following we study the mean of the top or antitop rapidity distribution as a function of the transverse momentum and the rapidity of the object radiated by partons (q: initial quarks and produced top) or by antipartons ( $\bar{q}$ : initial antiquarks and produced antitops). The generation of the process $q \bar{q} \rightarrow t \bar{t} j$ requires a cut on the transverse momentum of the radiated object to stay away from soft/collinear singularities. The cut-off for $p_{T}^{g}$ is set to $10 \mathrm{GeV}$. Figure 5.17 presents the rapidity distributions of the produced top and antitop quarks. As expected, they show a similar shift as for PyThIA, HERWIG and MC@NLO in the exclusive jet bins greater than four. The mean of the distribution for the top quarks is slightly below zero, $\mu_{y t} \sim-0.07$. For the antitop quarks the distribution is shifted into the positive direction, the mean constitutes $\mu_{y \bar{t}} \sim 0.07$.

Similar to the correlation studies above using PYTHIA, we investigate the behavior of the mean of the top-quark rapidity distribution for different transverse momenta $\left(p_{T}^{g}\right)$, and for different rapidities $y_{g}$ of the radiated object. Figure 5.18 shows the transverse momentum of objects radiated from the partons $p_{T}^{g}(q)$ (a) as well as from antipartons $p_{T}^{g}(\bar{q})(\mathrm{b})$. Most radiations have a transverse momentum up to $100 \mathrm{GeV} / c$.

In figure 5.19 the upper plots (a) and (b) present the correlation on the parton side, i.e. between the rapidity of the top quark and the transverse momentum of the radiated object off a parton. The lower ones, (c) and (d), show the according plots for the antitop quark and radiations off the antiparton side. On the left hand side the correlation plots of the rapidity $y$ versus transverse momentum $p_{T}^{g}$ of the radiated object are shown, while the plots on the right hand side show the means of the $y$ distribution as a function of $p_{T}^{g}$. The mean values are obtained from a Gaussian fit to the rapidity distribution in a certain $p_{T}^{g}$ bin. The means of the top-quark rapidity 


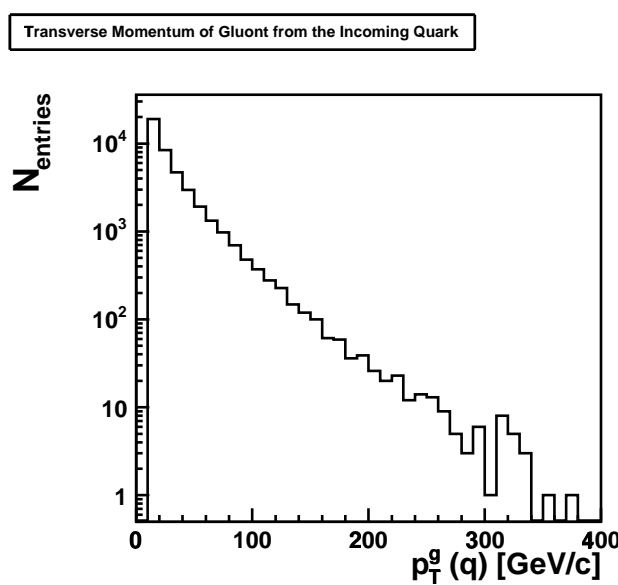

(a)

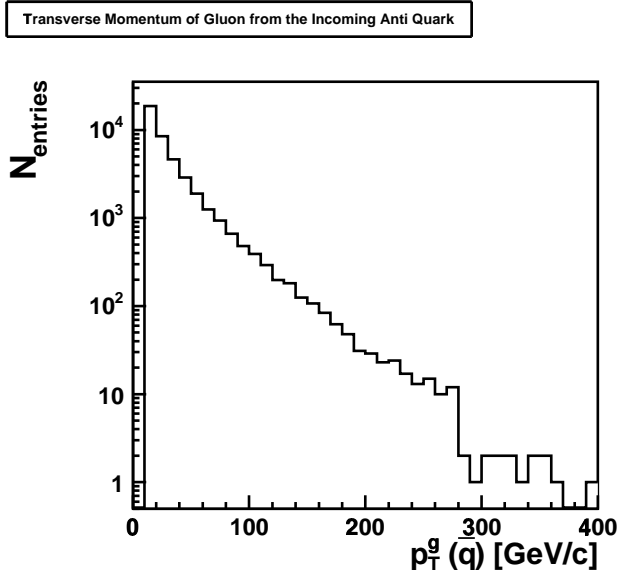

(b)

Figure 5.18: The distributions of the transverse momentum of gluons radiated from initial and final state quarks (a) as well as radiated from initial and final state antiquarks (b). The events are generated with MADEvent $(t \bar{t} j)$, where a cut on $p_{T}^{g}>10 \mathrm{GeV}$ is applied.

distributions (b) are slightly shifted to negative values for all $p_{T}^{g}$, where the absolute value of this shift seems to increase towards larger $p_{T}^{g}$. This increase is largest at small transverse momenta of the radiation. The corresponding effect is seen with opposite sign for the rapidity distributions of the antitop quark (d).

In figure 5.20 the corresponding results are presented if we consider the influence of the radiation rapidity. The upper plots (a) and (b) show the behavior of the top-quark rapidity distribution, the lower ones, (c) and (d), for the antitop quark. On the left hand side the correlation plots of rapidity $y$ versus rapidity $y_{g}$ of the radiated object are shown, while the plots on the right hand side show the means of the $y$ distribution as a function of $y_{g}$. Again, each mean value is obtained from a fit of a Gaussian to the rapidity distribution in a certain $y_{g}$ bin. The top rapidity distributions (b) are shifted to negative values for objects emitted centrally or in backward direction from both initial or final state quarks, while essentially no shift is seen for objects radiated into forward direction. In the case of the rapidity distribution of antitops $(d)$, where the object is radiated off the antipartons, this behavior is turned around.

The dependence of the asymmetry of the top and antitop quark on the transverse momentum and on the rapidity of the radiated gluon obtained with MADEvENT are very similar to the dependences obtained with the LO+PS Monte Carlo PyтHIA. 


\section{Rapidity vs Gluon hardness (q-t)}

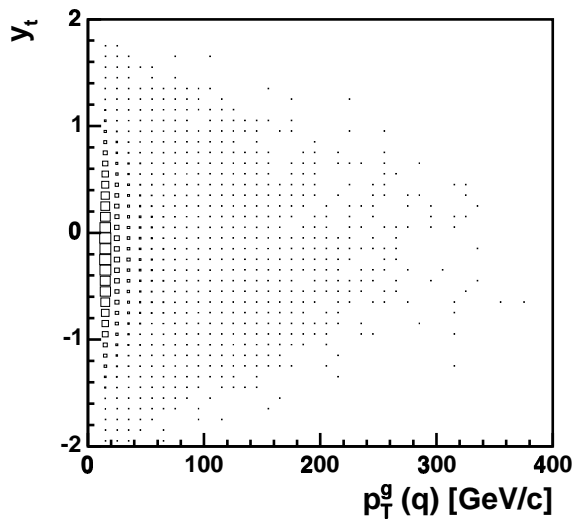

(a)

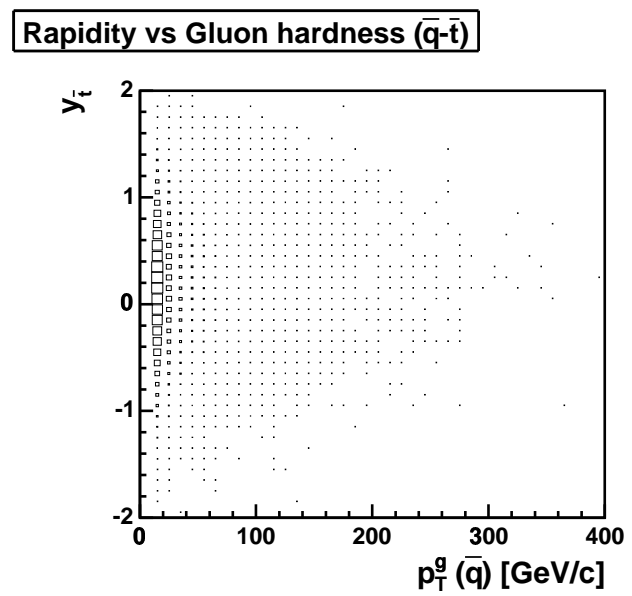

(c)

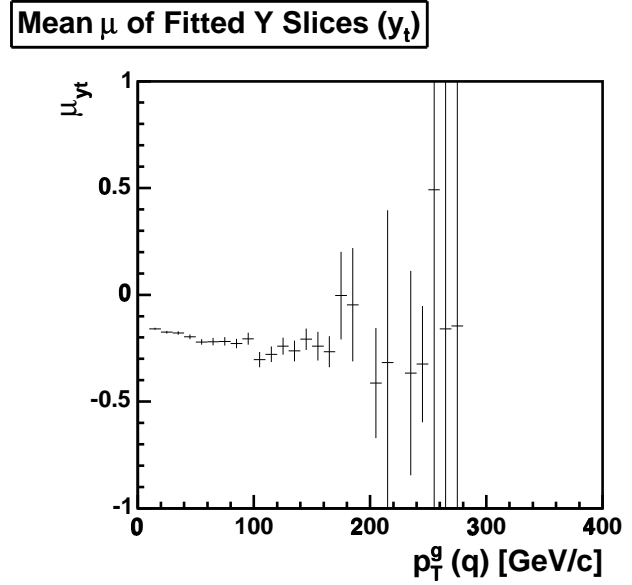

(b)

Mean $\mu$ of Fitted Y Slices $\left(y_{\bar{t}}\right)$

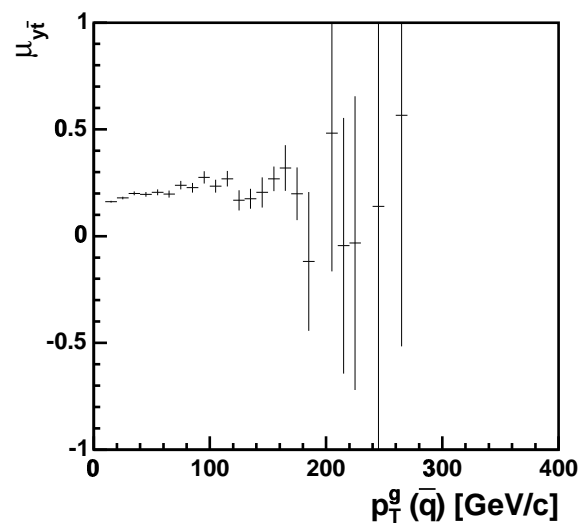

(d)

Figure 5.19: Correlation between the rapidity of the top (a, b) and the antitop quark (c, d) and the transverse momenta of the gluon radiated either from initial and final state quarks $(\mathrm{a}, \mathrm{b})$ or from initial and final state antiquarks (c, d). Left: Correlation plots rapidity $y$ versus transverse momentum $p_{T}^{g}$ of the radiated gluon; right: means of the $y$ distribution as a function of $p_{T}^{g}$. Here, each mean value is obtained from a fit of a Gaussian to the rapidity distribution in a certain $p_{T}^{g}$ bin. All distributions are obtained using MAdEvent $(t \bar{t} j)$ with a cut $p_{T}^{g}>10 \mathrm{GeV}$. 


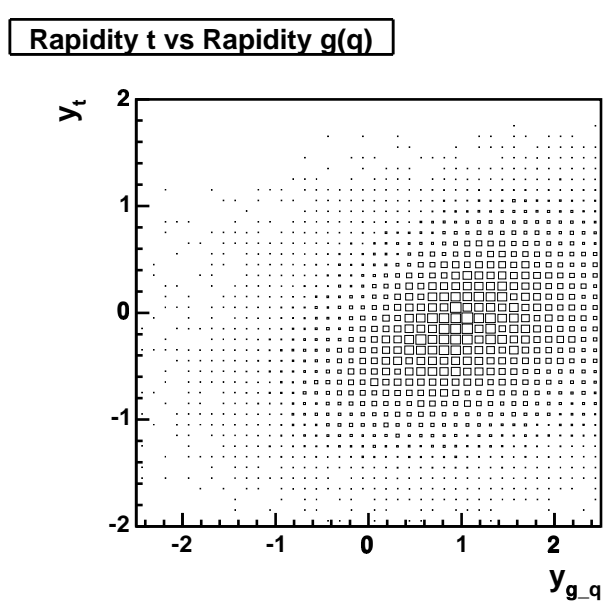

(a)

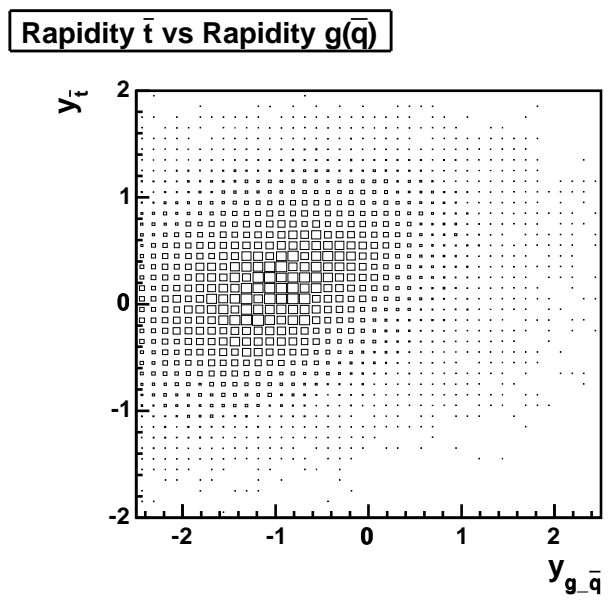

(c)

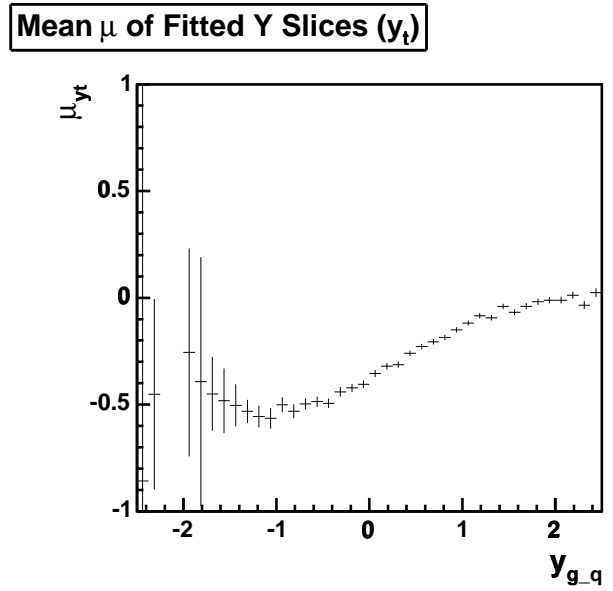

(b)

Mean $\mu$ of Fitted $Y$ Slices $\left(y_{\bar{t}}\right)$

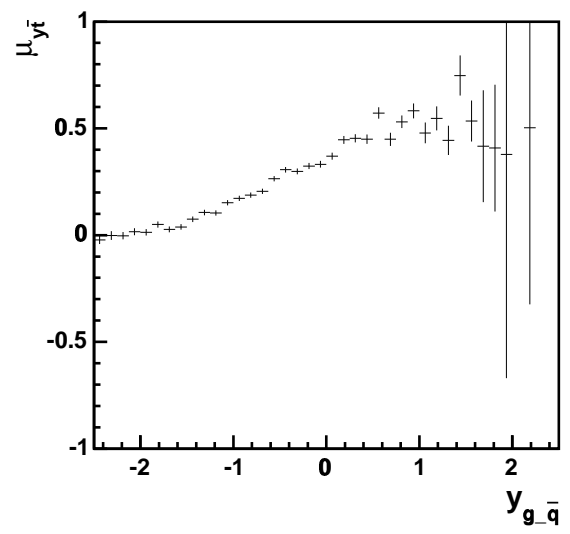

(d)

Figure 5.20: Correlation between the rapidity of the top (a, b) and the antitop quark (c, d) and the rapidity of the gluon radiated either from initial and final state quarks (a, b) or from initial and final state antiquarks $(\mathrm{c}, \mathrm{d})$. Left: Correlation plots rapidity $y$ versus rapidity $y_{g}$ of the radiated gluon; right: means of the $y$ distribution as a function of $y_{g}$. Here, each mean value is obtained from a fit of a Gaussian to the rapidity distribution in a certain $y_{g}$ bin. All distributions are obtained using MADEvent $(t \bar{t} j)$ with a cut $p_{T}^{g}>10 \mathrm{GeV}$. 


\subsection{Summary}

Studying the influence of the event selection on the charge asymmetry $A$ we observe a strong dependence of the asymmetry on the number of jets in the event. This effect is not only seen in NLO+PS Monte Carlo MC@NLO, but also in the LO+PS Monte Carlos Pythia and HeRwig. As a result of the event selection study we find, that cuts on the jet kinematics have a certain impact on the asymmetry. The more central and the harder the jets are required to be, the more negative becomes the observed asymmetry. This explains the shift of the inclusive asymmetry after applying all selection cuts to smaller values, which was the original reason for this Monte Carlo study. Since events with additional jets show a more negative asymmetry, further studies investigate the influence of gluon radiation on the rapidity distribution of the produced top quarks. Only top quarks produced via $q \bar{q}$ annihilation are affected by the jet bin dependence due to a color connection between the initial quarks and the produced top quarks. We also observe a dependence of the top-quark rapidity on the strength and direction of the radiation, which is consistent with the findings of the event selection study. 


\section{Chapter 6}

\section{Measurement of the Charge Asymmetry in Top-Pair Production}

In this chapter we present measurements of the asymmetry $A\left(\Delta y \cdot Q_{l}\right)$ in the inclusive $t \bar{t}$ sample $\left(N_{\text {jets }} \geq 4\right)$ as well as in the exclusive four- and five-jets samples. The MC studies in the previous chapter have shown, that a measurement of the asymmetry in an event sample with at least four jets contains different asymmetric effects. In pure $t \bar{t}$ production, the interference of different contributions leads to a slight positive asymmetry as described in chapter 1.3. Considering hard gluon radiation, the interference of the corresponding hard initial- and final-state gluon radiation leads to a negative asymmetry. In higher jet bins the contribution of hard gluon radiation events increases. The exclusive measurements in the four- and fivejets bin are a first attempt to separate the negative contribution to the asymmetry in top-pair production. In the following the different analysis steps are presented. First, the background contributions are discussed and an estimate of the rates is given. A method is developed to correct the measured rapidity difference for reconstruction and event-selection effects. This is done on the base of the PYTHIA Monte Carlo signal sample. Taking these effects and several background contributions into account, we perform a measurement of the asymmetry in the different $t \bar{t}$ jet samples obtained from the experiment. Finally, the systematic uncertainties of the results are estimated.

\subsection{Background Estimation}

Although the event selection accomplishes a background reduction up to a signal to background ratio of $S: B \sim 7: 1$, the extracted $t \bar{t}$ sample still contains certain types of background. One source of background events is $W$ production in association with heavy quark and light quark production. In the case of light quark production, one jet is misidentified as a $b$-quark jet (mistags). Events with one or more jets originating from a $c$ or $b$ quark are called " $W+$ heavy flavor" events. Furthermore, there are events in which no real $W$ boson is produced (non- $W$ background), e.g. 
direct $b \bar{b}$ production with additional gluon radiation. For this background category, one jet has to fake a charged lepton to overcome the tight lepton requirement of the event selection. In addition, electroweak processes like single $Z$ boson, diboson $(Z W, W W, Z Z)$ and single-top production contribute to the background. The fraction of these electroweak events is rather small and can be determined from their theoretical cross section and the acceptance and efficiencies derived from MC simulation and measured data.

Different methods are employed to model the background shape of differential distributions. The mistags and $W+$ heavy flavor contribution as well as the diboson and single-top background is determined from different Monte Carlo samples, listed in table 4.1. For the mistag background we use the $W+4 p$ sample ( $W$ plus four light quarks) and for the $W+$ heavy flavor background as well as for the diboson and single-top background we use the $W+2 p+2 b$ sample ( $W$ plus two light quarks plus two $b$ quarks). The shape of the non- $W$ background (QCD background) is modeled by a multi-jet data sample containing events with at least 5 jets, whereof one jet has to be misidentified as the required charged lepton. In this control data sample it is not possible to require one of the jets to be $b$ tagged because too few events would remain. Therefore, a special treatment is necessary. Unlike to the original event selection, all events considered as taggable are accepted to obtain sufficient statistics. The $b$-tag information is afterwards assigned randomly to one of the remaining jets in the event.

\begin{tabular}{c|c|c|c} 
& Inclusive & Exclusive $(4$ jets) & Exclusive $(5$ jets $)$ \\
\hline \hline$W+c$ & $0.60 \pm 0.15$ & - & - \\
$W+c \bar{c}$ & $3.04 \pm 0.95$ & - & - \\
$W+b \bar{b}$ & $6.38 \pm 1.80$ & - & - \\
Diboson & $1.63 \pm 0.29$ & - & - \\
Single-Top & $0.62 \pm 0.10$ & - & $1.61 \pm 0.32$ \\
\hline$\sum_{E W+H F}$ & $12.27 \pm 2.48$ & $10.28 \pm 2.08$ & $1.27 \pm 0.34$ \\
$\mathrm{QCD}($ non- $W)$ & $6.80 \pm 1.80$ & $5.31 \pm 1.41$ & $2.01 \pm 0.30$ \\
Mistags & $12.25 \pm 1.83$ & $9.51 \pm 1.42$ & $4.89 \pm 0.52$ \\
\hline \hline$N_{\text {tot }}^{B g}$ & $31.32 \pm 3.81$ & $25.1 \pm 3.16$ & \\
\hline \hline
\end{tabular}

Table 6.1: Overview of the background estimation for the different contributions. The $W+$ heavy flavor and the electroweak fractions are combined.

All those background samples, except the non- $W$ sample, are passed through the CDF detector simulation and afterwards the same reconstruction as for real data is applied. The correct normalization of the different contributions is received from the background estimation determined by reference [58]. The total number of expected background events in the inclusive $t \bar{t}$ sample is $31.32 \pm 3.81$. The estimates for each background contribution are listed in table 6.1. These estimates are only available for the inclusive case. To obtain the background numbers for the exclusive jet bins, we scale the inclusive estimate. The scaling factors for the different contributions are obtained from a comparison of the number of events in the three inclusive back- 
ground samples $\left(N_{\text {jets }} \geq 4\right)$ with the number of events in the four- and five-jets bin samples. Thus, we obtain a total background estimate of $25.1 \pm 3.16$ in the four-jets bin and $4.89 \pm 0.52$ in the five-jets bin. The estimates for the different contributions are listed in table 6.1 in the center and right column.

\subsection{Correction for Acceptance and Smearing Ef- fects}

An asymmetry calculated in the reconstructed measured $t \bar{t}$ sample does not reflect the initial value, due to detector acceptance, event selection and reconstruction effects that distort the true $\Delta y \cdot Q_{l}$ distribution. Since the asymmetry is given by the difference of events with $\Delta y \cdot Q_{l}>0$ and $\Delta y \cdot Q_{l}<0$, the rapidity distribution is partitioned into these two bins $p, n$ (positive, negative). Thus, the influence of the different analysis steps on the shape of $\Delta y \cdot Q_{l}$ is only interesting in view of effects that change the population of the two bins. A quantitative description of the impact of event selection and reconstruction on the number of positive and negative events is derived from the PyTHIA signal sample.

From the reconstructed $\Delta y \cdot Q_{l}$ distribution we obtain $N_{p}^{r e c}$ events with $\Delta y \cdot Q_{l}>0$, and $N_{n}^{r e c}$ events with $\Delta y \cdot Q_{l}<0$. In the following, a correction of these event numbers for detector acceptance and reconstruction effects is derived. Since this is a Monte Carlo based process, we start from the true $\Delta y \cdot Q_{l}$ distribution yielding the generated event numbers $N_{p}^{g e n}$ and $N_{n}^{g e n}$.

\section{Event Selection:}

The impact of the event selection on the $\Delta y \cdot Q_{l}$ distribution can be divided into mainly to aspects. The acceptance of $t \bar{t}$ events is take into account by the total efficiency $\epsilon_{t o t}=N^{s e l} / N^{g e n}$, representing the fraction of generated signal events, that pass the event selection. Since the detector acceptance may not be homogeneous over the observed $\Delta y \cdot Q_{l}$ range, it is reasonable to consider the efficiency in each bin of the distribution, $\epsilon_{i}=N_{i}^{\text {sel }} / N_{i}^{g e n}$. The relative efficiency is then given by:

$$
\epsilon_{r e l, i}=\frac{\epsilon_{i}}{\epsilon_{t o t}}=\frac{N_{i}^{s e l} / N^{s e l}}{N_{i}^{g e n} / N^{g e n}} .
$$

Thus, the number of observed events in a certain bin $i$ after the selection can be obtained from:

$$
N_{i}^{s e l}=\epsilon_{t o t} \cdot \epsilon_{r e l, i} \cdot N_{i}^{g e n} .
$$

In figure 6.1 the relative efficiencies of each bin of the $\Delta y \cdot Q_{l}$ distribution are presented. Comparing the resulting shapes of the three used MC signal samples there are no significant differences. In the case of the inclusive distribution, shown in plot (a), the relative efficiencies are distributed symmetrically around zero. The symmetric character of $\epsilon_{r e l, i}$ remains in the four-jets bin as can be seen in plot (b). In the event sample with five clustered jets, we obtain a quite asymmetric efficiency. 
The plot on the right hand side of figure 6.1 (c) shows, that events with a negative rapidity difference are more often accepted than events with positive $\Delta y \cdot Q_{l}$.

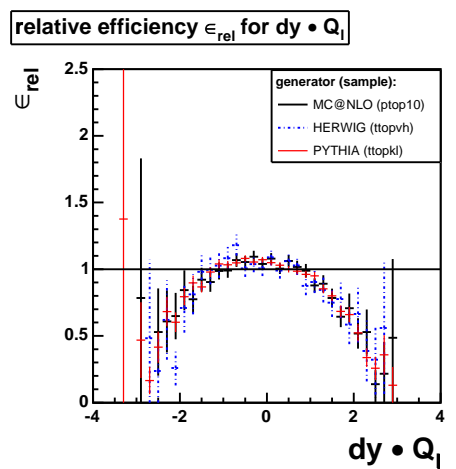

(a) Inclusive

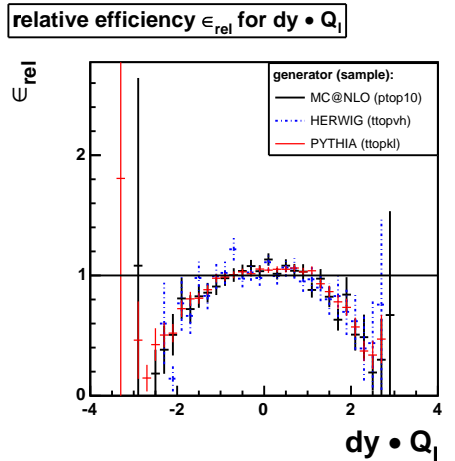

(b) Exclusive (4 jets)

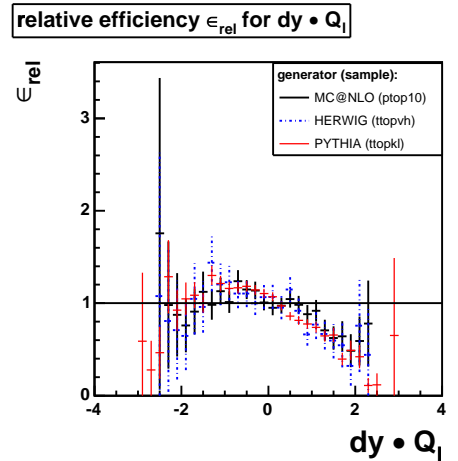

(c) Exclusive (5 jets)

Figure 6.1: Relative efficiencies of the $\Delta y \cdot Q_{l}$ distribution. Compared are the values for the three different Monte Carlo signal samples. The study is made in the inclusive sample (a), in the four-jets sample (b) and in the five-jets sample (c). One can see, that for $N_{\text {jets }}=4$ there is a slightly higher selection efficiency for events with a positive rapidity, whereas in the five-jets sample events with a negative $\Delta y \cdot Q_{l}$ are preferred.

The impact of the event selection on the population of the two bins $p$ and $n$ is described by the relative efficiencies $\epsilon_{p}$ and $\epsilon_{n}$ :

$$
\epsilon_{p}=\frac{N^{s e l}\left(\Delta y \cdot Q_{l}>0\right)}{N^{g e n}\left(\Delta y \cdot Q_{l}>0\right)} \cdot \frac{1}{\epsilon_{t o t}}, \quad \epsilon_{n}=\frac{N^{s e l}\left(\Delta y \cdot Q_{l}<0\right)}{N^{g e n}\left(\Delta y \cdot Q_{l}<0\right)} \cdot \frac{1}{\epsilon_{t o t}} .
$$

We have seen, that the choice of the $\mathrm{MC}$ event generator has no significant influence on the distribution of the relative efficiency, although the initial asymmetry is different in in the MC@NLO sample. Due to the similarity of the shapes, we are able to use the Pythia $t \bar{t}$ sample for the calculation of the efficiencies $\epsilon_{p}$ and $\epsilon_{n}$. The resulting values are listed below in table 6.2 .

\section{Reconstruction of the Top-Quark Pair:}

The migration of events from one bin to another due to uncertainties in the reconstruction of the top-quark four-vectors is described by the smearing matrix $S_{i k}$. The matrix elements give the probability for an event which was generated in bin $i$ of the Monte Carlo generated $\Delta y \cdot Q_{l}$ distribution to occur in bin $k$ of the reconstructed one. Since our measurement considers only the two bins $p$ and $n$, the smearing matrix $S$ is given by:

$$
S=\left(\begin{array}{cc}
S_{p p} & S_{n p} \\
S_{p n} & S_{n n}
\end{array}\right)
$$




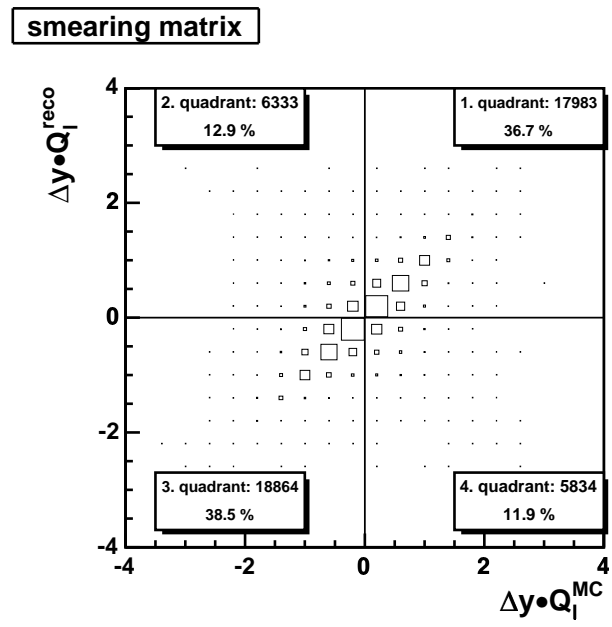

(a) Inclusive

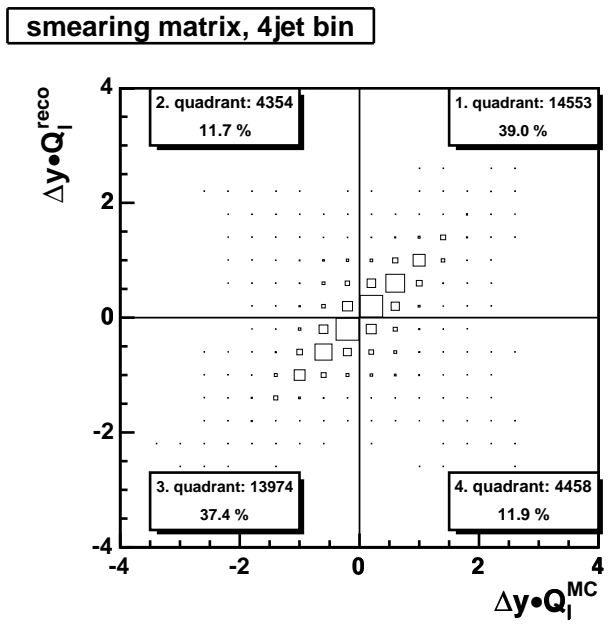

(b) Exclusive (4 jets)

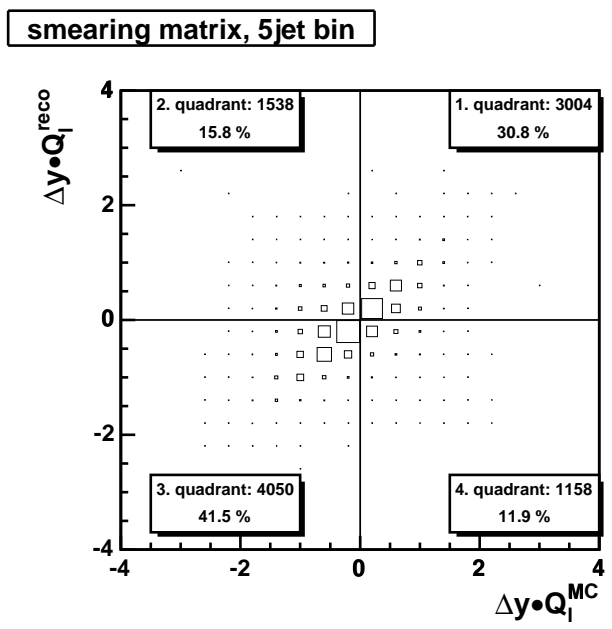

(c) Exclusive (5 jets)

Figure 6.2: Migration plots of $\Delta y \cdot Q_{l}$ for the inclusive PYTHIA samples (a) as well as for the exclusive cases ((b) $N_{\text {jets }}=4$, (c) $\left.N_{\text {jets }}=5\right)$. The interpretation of the content of different quadrants is given in the text.

The matrix elements are obtained from the comparison of the $\Delta y \cdot Q_{l}^{M C}$ distribution of Monte Carlo signal events after passing the event selection with the distribution of these events after they were reconstructed $\left(\Delta y \cdot Q_{l}^{\text {reco }}\right)$. This is done using the Pyтhia $t \bar{t}$ sample. The corresponding two dimensional plots are presented in figure 6.2. The distribution for the inclusive $t \bar{t}$ sample is shown in (a), the one for the four-jets bin in (b), and the distribution for the five-jets bin in (c). The first (third) quadrant always contains events, for which the rapidity difference remains 
positive (negative) during the reconstruction process. But there are also events, where the reconstruction of the top-quark four-vectors changes the sign of the rapidity difference. Thus, they migrate from the positive into the negative bin and v.v. Since this flow of events is not equal in both directions, an artificial asymmetry is generated which has to be corrected. Because all events of bin $i$ have to occur somewhere in the reconstructed $\Delta y \cdot Q_{l}$ distribution, the matrix elements $S_{i k}$ are defined in such a way, that $\sum_{k} S_{i k}=1$ with $i(k)=p, n$ holds for all bins $i$ :

$$
S_{p p}=\frac{N_{p p}}{N_{p p}+N_{p n}}, \quad S_{p n}=\frac{N_{p n}}{N_{p p}+N_{p n}}, \quad S_{n p}=\frac{N_{n p}}{N_{n p}+N_{n n}}, \quad S_{n n}=\frac{N_{n n}}{N_{n p}+N_{n n}}
$$

The precise values of the matrix elements for the inclusive and the exclusive case can be found in table 6.2. A comparison of the smearing matrix elements obtained from the different MC generators shows, that $S$ is independent of the initial asymmetry. Thus, we decide to use the matrix based on the PyTHIA signal to correct the measured data.

\section{Correction Matrix $C$ :}

Accounting for the effects of event selection and reconstruction in the discussed way, it is possible to relate the true event numbers $N_{p}^{g e n}$ and $N_{n}^{g e n}$ of the generated MC signal sample to the remaining, fully reconstructed numbers $N_{p}^{r e c o}$ and $N_{n}^{r e c o}$. This relation is given by:

$$
\left(\begin{array}{c}
N_{p}^{\text {reco }} \\
N_{n}^{\text {reco }}
\end{array}\right)=\epsilon_{\text {tot }} \cdot\left(\begin{array}{cc}
\epsilon_{p} & 0 \\
0 & \epsilon_{n}
\end{array}\right) \cdot\left(\begin{array}{cc}
S_{p p} & S_{n p} \\
S_{p n} & S_{n n}
\end{array}\right) \cdot\left(\begin{array}{c}
N_{p}^{g e n} \\
N_{n}^{g e n}
\end{array}\right)
$$

Introducing the matrix $C$ :

$$
C=\left(\begin{array}{cc}
\epsilon_{p} & 0 \\
0 & \epsilon_{n}
\end{array}\right) \cdot\left(\begin{array}{cc}
S_{p p} & S_{n p} \\
S_{p n} & S_{n n}
\end{array}\right)=\left(\begin{array}{cc}
\epsilon_{p} \cdot S_{p p} & \epsilon_{p} \cdot S_{n p} \\
\epsilon_{n} \cdot S_{p n} & \epsilon_{n} \cdot S_{n n}
\end{array}\right)
$$

equation (6.6) leads to:

$$
\left(\begin{array}{c}
N_{p}^{\text {reco }} \\
N_{n}^{\text {reco }}
\end{array}\right)=\epsilon_{t o t} \cdot\left(\begin{array}{cc}
C_{p p} & C_{n p} \\
C_{p n} & C_{n n}
\end{array}\right) \cdot\left(\begin{array}{c}
N_{p}^{g e n} \\
N_{n}^{g e n}
\end{array}\right)
$$

Because the asymmetry is independent of a constant factor, $\epsilon_{\text {tot }}$ has no impact on the asymmetry and can thus be absorbed by defining $\hat{N}_{p}=N_{p}^{g e n} \cdot \epsilon_{\text {tot }}$ and $\hat{N}_{n}=N_{n}^{g e n} \cdot \epsilon_{\text {tot }}$. Equation (6.8) then modifies to:

$$
\left(\begin{array}{c}
N_{p}^{\text {reco }} \\
N_{n}^{\text {reco }}
\end{array}\right)=\left(\begin{array}{ll}
C_{p p} & C_{n p} \\
C_{p n} & C_{n n}
\end{array}\right) \cdot\left(\begin{array}{c}
\hat{N}_{p} \\
\hat{N}_{n}
\end{array}\right)
$$

Knowing the initial event distribution, equations (6.6-6.9) provide the corresponding distribution after selection and reconstruction.

In the experiment the situation is converse. We start with the reconstructed, background subtracted event numbers $N_{p}^{B g S u b}$ and $N_{n}^{B g S u b}$, corresponding to the 


\begin{tabular}{c|c|c|c|c|c|c} 
& \multicolumn{2}{|c|}{ Inclusive } & \multicolumn{2}{c|}{ Exclusive $(4$ jets $)$} & \multicolumn{2}{c}{ Exclusive (5 jets) } \\
& PYTHIA & MC@NLO & PYTHIA & MC@NLO & PYTHIA & MC@NLO \\
\hline \hline$\epsilon_{p}$ & 0.97 & 0.98 & 1.02 & 1.01 & 0.85 & 0.91 \\
$\epsilon_{n}$ & 1.03 & 1.02 & 0.98 & 0.99 & 1.15 & 1.09 \\
\hline$S_{p p}$ & 0.755 & 0.745 & 0.765 & 0.756 & 0.721 & 0.725 \\
$S_{n p}$ & 0.251 & 0.265 & 0.237 & 0.255 & 0.275 & 0.288 \\
$S_{p n}$ & 0.245 & 0.255 & 0.235 & 0.244 & 0.279 & 0.275 \\
$S_{n n}$ & 0.749 & 0.735 & 0.763 & 0.745 & 0.725 & 0.721 \\
\hline$C_{p p}^{-1}$ & 1.535 & 1.567 & 1.423 & 1.472 & 1.909 & 1.795 \\
$C_{n p}^{-1}$ & -0.516 & -0.511 & -0.443 & -0.504 & -0.725 & -0.725 \\
$C_{p n}^{-1}$ & -0.472 & -0.473 & -0.451 & -0.490 & -0.542 & -0.579 \\
$C_{n n}^{-1}$ & 1.452 & 1.456 & 1.471 & 1.521 & 1.407 & 1.528 \\
\hline \hline
\end{tabular}

Table 6.2: Efficiencies $\epsilon_{p / n}$, smearing matrix elements $S(i, k)$, and correction matrix elements $C^{-1}(i, k)$. They are determined with PYтнIA for the inclusive analysis as well as for the analysis performed in the four- and five-jets bins. The uncertainties are small and thus not listed here.

"reco" event numbers in equation 6.9, from which we need to extract the initial event numbers $\hat{N}_{p}$ and $\hat{N}_{n}$. This can be achieved by multiplying equation 6.9 with the inverted matrix $C^{-1}$. The initial events numbers in the two bins $p, n$ are then obtained from the reconstructed event numbers by:

$$
\begin{aligned}
\left(\begin{array}{c}
\hat{N}_{p} \\
\hat{N}_{n}
\end{array}\right) & =C^{-1} \cdot\left(\begin{array}{c}
N_{p}^{\text {reco }} \\
N_{n}^{\text {reco }}
\end{array}\right) \\
& =\frac{1}{C_{p p} \cdot C_{n n}-C_{n p} \cdot C_{p n}} \cdot\left(\begin{array}{cc}
C_{n n} & -C_{n p} \\
-C_{p n} & C_{p p}
\end{array}\right)\left(\begin{array}{c}
N_{p}^{\text {reco }} \\
N_{n}^{\text {reco }}
\end{array}\right)
\end{aligned}
$$

The resulting matrix elements of the inverted matrix obtained from the PYTHIA signal sample are given in table 6.2. By applying this correction matrix $C^{-1}$ to the background subtracted event numbers $N_{p}^{B g S u b}$ and $N_{n}^{B g S u b}$ of the measured $\Delta y \cdot Q_{l}$ distribution, it is possible to extract the true event numbers. Thus, we are able to calculate the asymmetry $A\left(\Delta y Q_{l}\right)$ in the unfolded distribution:

$$
A\left(\Delta y Q_{l}\right)=\frac{\hat{N}_{p}-\hat{N}_{n}}{\hat{N}_{p}+\hat{N}_{n}},
$$

which can now also be compared to theoretical predictions.

\subsection{Inclusive and Exclusive Measurement}

We analyze a $t \bar{t}$ data sample corresponding to a total integrated luminosity of $955 \mathrm{pb}^{-1}$, which is divided into three electron and three muon samples. They con- 
tain, amongst others, information about the final state particles as they are reconstructed by data processing, i.e. the charged lepton, missing transverse energy, and all observed jets. After applying the event selection, we fully reconstruct the topquark four-vectors based on the information contained in the measured data sample. Using the reconstructed top-quark kinematics, the rapidity difference $\Delta y \cdot Q_{l}$ is calculated. In figure 6.3 the $\Delta y \cdot Q_{l}$ distributions for the inclusive and the exclusively reconstructed data samples are presented.

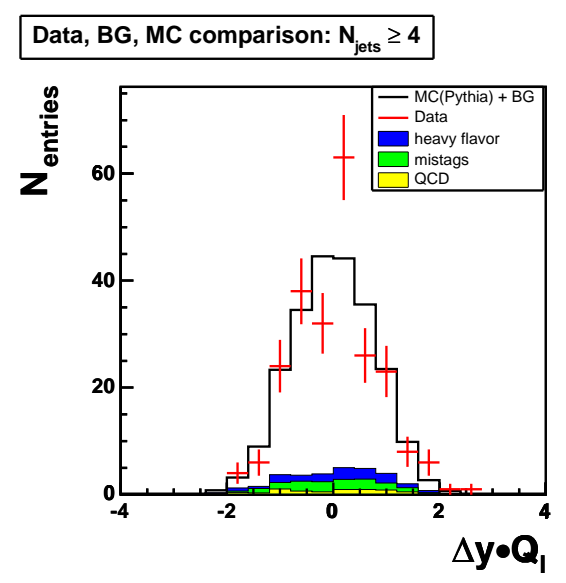

(a)

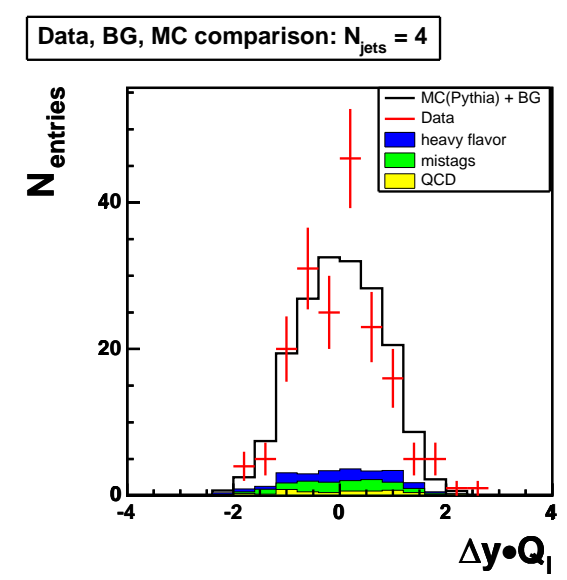

(b)

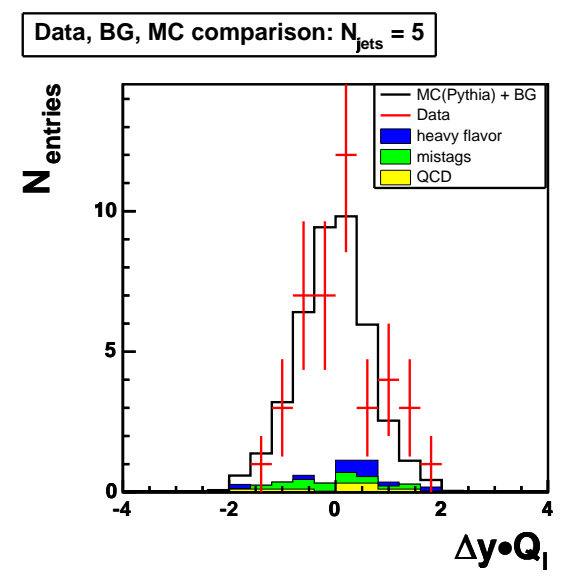

(c)

Figure 6.3: Comparison of the $\Delta y \cdot Q_{l}$ distribution measured in data (red crosses) with the MC expectation generated with PYTHIA plus background estimation.

This distribution, represented by the red crosses, is compared to the reconstructed Pyтhia Monte Carlo expectation, to which the different background contributions are added. We observe, that the distribution of the background fraction is symmetric in the inclusive sample, (a), as well as in the exclusive four-jets sample, 
(b). In the five-jets sample, (c), the heavy flavor and the QCD fractions are mainly cumulated in the positive region of $\Delta y \cdot Q_{l}$.

\begin{tabular}{c|c|c|c} 
& Inclusive & Exclusive $(4$ jets $)$ & Exclusive $(5$ jets $)$ \\
\hline \hline Reconstructed: & & & 7 \\
bhel0d & 48 & 38 & 11 \\
bhel0h & 51 & 32 & 7 \\
bhel0i & 42 & 20 & 3 \\
bhmu0d & 36 & 26 & 6 \\
bhmu0h & 32 & 26 & 7 \\
bhmu0i & 23 & 182 & 42 \\
$N^{\text {tot }}$ & 232 & 97 & 23 \\
$N_{p}$ & 128 & 85 & 18 \\
$N_{n}$ & 104 & $0.06 \pm 0.074$ & $0.12 \pm 0.155$ \\
\hline$A^{\text {rec }}\left(\Delta y \cdot Q_{l}\right)$ & $0.10 \pm 0.065$ & & 3.1 \\
\hline Background subtracted: $_{p}^{\text {Bg }}$ & & 17.95 & 1.8 \\
$N_{n}^{B g}$ & 14.32 & 12.15 & $19.9 \pm 4.5$ \\
$N_{p}^{B g S u b}$ & $111 \pm 10.5$ & $84.05 \pm 9.2$ & $16.2 \pm 4.0$ \\
$N_{n}^{B g S u b}$ & $89.68 \pm 9.5$ & $72.85 \pm 8.5$ & $0.10 \pm 0.176$ \\
\hline$A^{\text {BgSub }}\left(\Delta y \cdot Q_{l}\right)$ & $0.11 \pm 0.075$ & $0.07 \pm 0.086$ & \\
\hline Corrected: & & & $23.5 \pm 10.3$ \\
$\hat{N}_{p}$ & $123.40 \pm 19.3$ & $83.75 \pm 15.5$ & $12.6 \pm 6.8$ \\
$\hat{N}_{n}$ & $77.28 \pm 16.8$ & $73.15 \pm 15.3$ & $0.37 \pm 0.30$ \\
\hline \hline$A\left(\Delta y \cdot Q_{l}\right)$ & $0.23 \pm 0.12$ & $0.11 \pm 0.14$ & \\
\hline \hline
\end{tabular}

Table 6.3: Overview of the number of $t \bar{t}$ events following the order of the analysis. The numbers are given for the inclusive sample, as well as for the exclusive four- and five-jets samples. First, the number of $t \bar{t}$ events in the used electron and muon data samples are listed. The estimated background events in the bins $p$ and $n$ lead to the background subtracted event numbers, that can then be corrected to obtain the true numbers $\hat{N}_{p}$ and $\hat{N}_{n}$. Based on these the asymmetry is calculated. The statistical uncertainties of the event numbers in the first section ("reconstructed") are the Poisson errors of the event numbers. The calculation of the uncertainties of the background subtracted and corrected event numbers can be found in appendix A. The statistical uncertainty of the asymmetry is then obtained from equation 6.13 .

The number of observed $t \bar{t}$ candidate events in the different reconstructed data samples (inclusive, four- and five jets samples) are listed in table 6.3. The table is organized in three sections: the first part gives the observed number of events. The $\Delta y \cdot Q_{l}$ distributions of the background, scaled to the expected rate, deliver the positive and negative background event numbers which have to be subtracted from the reconstructed numbers $N_{p}$ and $N_{n}$. Figure 6.4 shows a comparison of the exclusive asymmetry calculated using the background subtracted event numbers with the expectation of the different MC signal samples. With the obtained background subtracted numbers, the correction can be applied to receive the true numbers $\hat{N}_{p}$ 


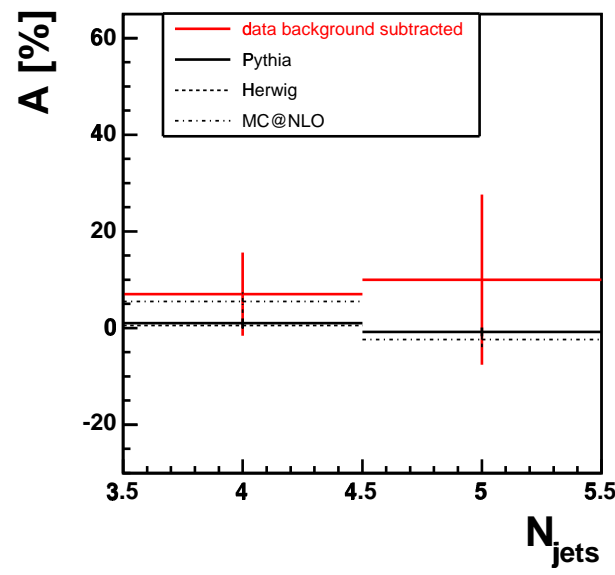

Figure 6.4: Comparison of asymmetry of the background subtracted event numbers with the MC expectation in the exclusive four- and five-jets bins. The solid, red crosses show the asymmetry extracted from the background subtracted event numbers. The black lines represent the MC expectations, whereby the solid line represents the PүтнIA, the dashed one the HERWIG, and the dash-dotted one the MC@NLO signal sample. Since the predictions of the leading-order MC samples PYTHIA and HERWIG are similar, they are hard to distinguish in the plot.

and $\hat{N}_{n}$ according to:

$$
\begin{gathered}
\left(\begin{array}{c}
\hat{N}_{p} \\
\hat{N}_{n}
\end{array}\right)_{i n c .}=C_{i n c .}^{-1} \cdot\left(\begin{array}{c}
111 \\
89.68
\end{array}\right), \\
\left(\begin{array}{c}
\hat{N}_{p} \\
\hat{N}_{n}
\end{array}\right)_{4}=C_{4}^{-1} \cdot\left(\begin{array}{c}
84.05 \\
72.85
\end{array}\right), \\
\left(\begin{array}{c}
\hat{N}_{p} \\
\hat{N}_{n}
\end{array}\right)_{5}=C_{5}^{-1} \cdot\left(\begin{array}{c}
19.9 \\
16.2
\end{array}\right) .
\end{gathered}
$$

Based on these corrected event numbers the asymmetry in the different samples can be calculated using equation 6.11. The statistical uncertainties of the asymmetry are calculated by Gaussian error propagation:

$$
\sigma_{A}=\left[\left(\frac{2 \hat{N}_{n}}{\left(\hat{N}_{n}+\hat{N}_{p}\right)^{2}}\right)^{2} \cdot \sigma_{\hat{N}_{p}}^{2}+\left(\frac{2 \hat{N}_{p}}{\left(\hat{N}_{n}+\hat{N}_{p}\right)^{2}}\right)^{2} \cdot \sigma_{\hat{N}_{n}}^{2}\right]^{1 / 2},
$$

whereby $\hat{N}_{p}$ and $\hat{N}_{n}$ depend on the efficiencies $\epsilon_{p} / \epsilon_{n}$ and on the smearing matrix elements $S_{i k}$. The uncertainties due to the correction matrix are again taken into account by Gaussian error propagation, at which those of the efficiencies and of the smearing matrix elements are described by binomial errors. The complete error propagation can be found in appendix A. The statistical error of an asymmetry 
depends generally on the measured value. This can be illustrated by rewriting equation 6.13 and expressing $\sigma_{A}$ as a function of the number of $\hat{N}_{p}$ :

$$
\sigma_{A}\left(\hat{N}_{p}\right)=\frac{1}{2} \cdot\left[1-\left(\frac{2 \hat{N}_{p}}{\hat{N}}-1\right)^{2}\right] \cdot \sqrt{\frac{1}{\hat{N}_{p}}+\frac{1}{\hat{N}-\hat{N}_{p}}},
$$

whereby $\hat{N}=\hat{N}_{p}+\hat{N}_{n}$, and the statistical uncertainty of the positive and negative event numbers is given by the Poisson error.

Figure 6.5 visualizes exemplarily the quadratic shape of the statistical uncertainty. It is always dominated by the larger fraction of events. The error becomes maximal for an asymmetry of zero, when the number of positive and negative events is equal. If the absolute value of the asymmetry increases, either $N_{p}$ or $N_{n}$ becomes larger and the statistical uncertainty is reduced.

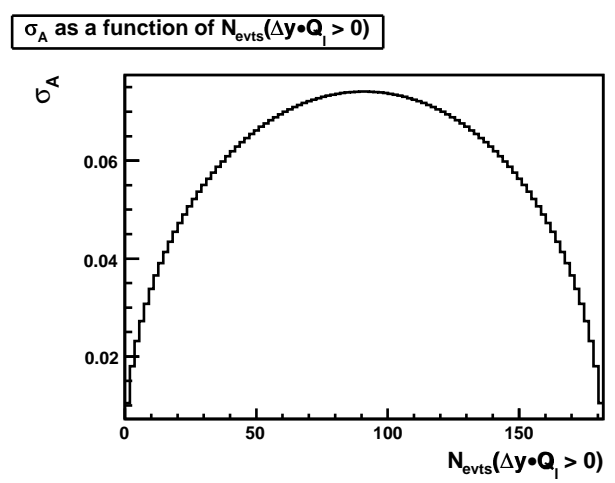

Figure 6.5: Statistical uncertainty $\sigma_{A}$ as a function of $N_{\text {evts }}\left(\Delta y \cdot Q_{l}>0\right)=N_{p} . N_{p}$ is varied in a range from zero to the total number of events $N_{t \bar{t}}^{t o t}$ in the four-jets sample.

The measured asymmetry in the different $t \bar{t}$ samples with the according statistical uncertainty amount to:

$$
\begin{aligned}
& A_{\text {inc }}\left(\Delta y \cdot Q_{l}\right)=0.23 \pm 0.12(\text { stat }) \\
& A_{4}\left(\Delta y \cdot Q_{l}\right)=0.11 \pm 0.14 \text { (stat) } \\
& A_{5}\left(\Delta y \cdot Q_{l}\right)=0.37 \pm 0.30 \text { (stat) }
\end{aligned}
$$

In the $t \bar{t}$ sample containing events with four observed jets, we measure an asymmetry of $0.11 \pm 0.14$ (stat). The statistical error is still dominant, but the exclusively measured asymmetry in the four-jets bin is in agreement with the next-to-leading order Monte Carlo expectation of about $\sim 5 \%$ (see figure 6.6). In the five-jets sample we obtain an asymmetry of $0.37 \pm 0.30$ (stat). On the basis of the MC studies performed in chapter 5 , we would have expected a slightly negative asymmetry. Due to the low statistics in the five-jets bin the measured value is statistically not significant. In the inclusive sample containing $t \bar{t}$ events with at least four jets, the measured asymmetry is $0.23 \pm 0.12$ (stat).

The exclusive values of the asymmetry in the four- and five-jets bins are illustrated in figure 6.6. They are compared to the simulated Monte Carlo expectation of 
the different signal samples generated with PYTHIA, HERWIG, and MC@NLO, which are the same as in figure 6.4.

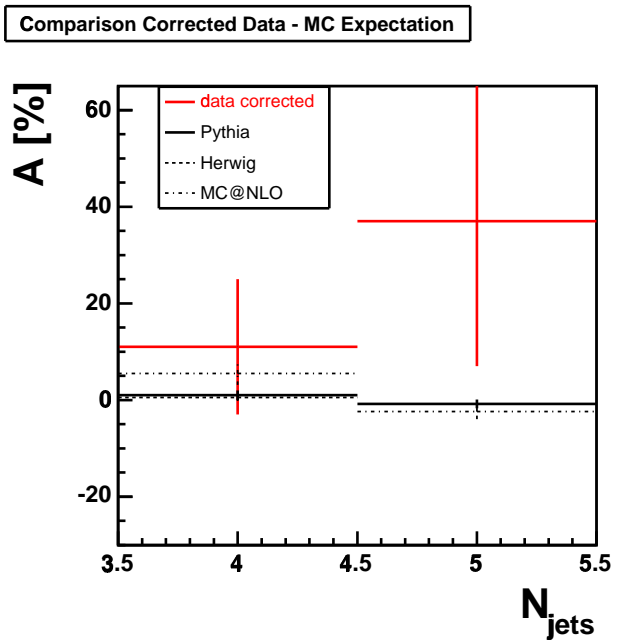

Figure 6.6: Comparison of measured exclusive results to the corresponding MC predictions in the four- and five-jets bin. The solid, red crosses show the asymmetry extracted from measured data. The black lines represent the MC expectations, whereby the solid line represents the PYтнIA, the dashed one the HERWIG, and the dash-dotted one the MC@NLO signal sample. Since the predictions of the leading-order MC samples PYTHIA and HERWIG are similar, they are hard to distinguish in the plot.

\subsection{Systematics}

Systematic uncertainties arise from uncertainties of theoretical parameters as well as from uncertainties concerning the experimental set up and the analysis method. Different contributions have been studied using pseudo experiments. Since the results of pseudo experiments fluctuate around the statistical uncertainty of the real measurement, performing series of pseudo experiments provides a statistically almost independent estimate of the behavior of a measurement method. This allows to study the influence of systematic modifications on the measured asymmetry. Therefore, we need to simulate the observed signal as well as the expected background events. The expected event numbers are thrown randomly according to a Poisson distribution whose mean corresponds to the expected event number from the real experiment. The means of the background fractions are the estimated ones listed in table 6.1, the mean for the signal events is then $N^{t o t}-N_{t o t}^{B g}$. The arrangement of the thrown events in a $\Delta y \cdot Q_{l}$ distribution is done based on the corresponding $\mathrm{MC}$ signal and background templates.

If we want to study the impact of a certain systematic effect on the measured asymmetry, the default signal sample is replaced by a MC sample generated with settings modified in a way, that corresponds to the studied systematic effect. A pseudo experiment is only considered, if the sum of thrown signal and background 
events corresponds to the expected number of observed data events $N^{\text {tot }}$. On each randomly generated $\Delta y \cdot Q_{l}$ distribution, we apply the full correction method. Efficiencies and smearing matrix described above in section 6.2 obtained from the default Pyтнia signal sample are applied to the simulated experiment.

Thus, we obtain from each pseudo experiment an asymmetry value and its statistical uncertainty. The mean of the asymmetry distribution $\mu_{A}^{i}$, obtained by performing several pseudo experiments on the base of different MC signal templates $i$, can be compared to each other. The variation of the mean is then a measure for the impact of the corresponding systematic uncertainty source on the extracted asymmetry.

Considering systematic uncertainties, we distinguish between sources due to theoretical parameters, and uncertainties due to the experimental setup and analysis method. Theoretical uncertainties arise during the MC event generation due to uncertainties of the used parameters. The different contributions taken into account by this analysis are:

\section{- MC Generator:}

The choice of a certain MC event generator may have an influence on the measured asymmetry, due to differences in the modeling of $t \bar{t}$ events. The impact is estimated by comparing the result $\mu_{A}$ if using a HERWIG signal sample to the one obtained from the default Pythia signal sample, both of which use a top-quark mass of $m_{t}=175 \mathrm{GeV} / \mathrm{c}^{2}$.

\section{- Parton Distribution Function:}

The impact of the PDF uncertainty is determined by using MC signal samples generated with different underlying PDFs. We compare MC signal samples, that use MRST72 and MRST75 [59] respectively for the generation, to a PythiA signal sample using CTEQ5L [60]. They were all generated with a top-quark mass of $m_{t}=178 \mathrm{GeV} / \mathrm{c}^{2}$. The systematic effect is then taken as the larger of the two deviations from the default value.

\section{- Top-Quark Mass:}

To estimate the influence of a varied top-quark mass we compare the two PythiA signal samples generated with a top-quark mass of $m_{t}=178 \mathrm{GeV} / \mathrm{c}^{2}$ and $m_{t}=175 \mathrm{GeV} / \mathrm{c}^{2}$ respectively.

\section{- Initial and Final State Radiation:}

The impact of ISR and FSR is estimated by using PyTHIA signal samples in which the parameters for the gluon radiation are varied to produce more or less ISR or FSR compared to the standard setup. The systematic uncertainty is given by the difference of the results obtained for the settings of more and less ISR/FSR.

The systematic uncertainties arising from theoretical uncertainties are summarized in the upper part of table 6.4. We study the following systematic uncertainties caused by the experimental setup and analysis method: 


\begin{tabular}{l|cc|cc|cc} 
& \multicolumn{2}{|c|}{ Inclusive } & \multicolumn{2}{c|}{ Exclusive (4 jets) } & \multicolumn{2}{c}{ Exclusive (5 jets) } \\
Source & $-\Delta A_{\text {inc. }}$ & $+\Delta A_{\text {inc. }}$ & $-\Delta A_{4}$ & $+\Delta A_{4}$ & $-\Delta A_{5}$ & $+\Delta A_{5}$ \\
\hline \hline MC gen. & 0.0001 & -0.0001 & - & - & - & - \\
PDF & 0.0308 & -0.0308 & - & - & - & - \\
Top-mass & 0.0100 & -0.0100 & 0.0265 & -0.0265 & 0.0102 & -0.0102 \\
ISR & 0.0079 & -0.0079 & - & - & - & - \\
FSR & 0.0321 & -0.0321 & - & - & - & - \\
\hline$E_{\text {Jet }}$ scale & 0.0084 & -0.0049 & 0.0103 & -0.0022 & 0.0032 & -0.0269 \\
BG, rate & 0.0160 & -0.0291 & 0.0050 & 0.0000 & 0.0529 & -0.0334 \\
BG, shape & 0.0302 & -0.0161 & 0.0212 & -0.0212 & 0.0517 & -0.0486 \\
\hline Total & 0.056 & -0.057 & 0.036 & -0.034 & 0.075 & -0.066 \\
\hline \hline
\end{tabular}

Table 6.4: Summary of systematic uncertainties, the total error is calculated by adding all single uncertainties in quadrature. In the exclusive case, some sources become part of the measurement and therefore are not taken into account as systematic uncertainty.

\section{- Jet Energy Scale (JES):}

The reconstructed $\Delta y \cdot Q_{l}$ distribution is altered by the jet energy corrections, since a modification of the jet energy scale changes the selected events and their reconstruction. The impact is estimated by processing the default PYTHIA sample with a jet correction varied within one standard deviation in both the negative (JES-) and the positive (JES + ) direction. The resulting $\mu_{A}( \pm)$ are compared to the one obtained from the PYтніA signal sample processed with the default jet energy corrections.

\section{- Background Rate:}

The quantitative influence of the background uncertainty is obtained from a series of pseudo experiments based on modified background rates. The Poisson means of all the different background contributions are simultaneously enhanced or reduced within one standard deviation, whereby the number of thrown data events remains unchanged. The results are compared to the default.

\section{- Background Shape Modeling:}

To check the uncertainty due to the background shape modeling, we use each shape of the three background distributions alone and simulate the total number of background events, instead of distributing them based on a composition of the three templates. The uncertainty obtained from the case with the largest deviation in the extracted asymmetry compared to the values obtained from the default background composition is then stated as systematic uncertainty.

The determined uncertainties of these sources are summarized in the lower part of table 6.4. By adding all single uncertainties in quadrature we obtain a total systematic uncertainty of +0.056 and -0.057 for the inclusive measurement of the asymmetry. For the measurement in the exclusive four- and five-jets bins one has to consider, that the measured asymmetry is due to gluon radiation effects and its 
theoretical description. This means, that certain effects considered in the inclusive case as a systematic uncertainty, become part of the measurement content in the exclusive samples. Thus, several sources listed in table 6.4 are not specified for the exclusive cases. First of all, ISR and FSR are rather a reason for the observed jet bin effect than a systematic effect. The Monte Carlo studies of the asymmetry in top-pair production have shown, that the parton shower modeling is able to simulate gluon radiation up to some limit. A variation of the PDF involves a variation of the QCD scale which also has an impact on gluon radiation modeling. Thus, these issues are not taken into account as systematic effects. The remaining contributions are again added in quadrature. This yields a systematic uncertainty of +0.036 and -0.034 for the asymmetry measured in the four-jets sample, while a total systematic uncertainty of +0.075 and -0.066 is determined for the measurement in the 5 jet bin. 
94Chapter 6. Measurement of the Charge Asymmetry in Top-Pair Production 


\section{Chapter 7}

\section{Conclusion and Outlook}

In this thesis I have presented the measurement of the charge asymmetry in top-pair production, analyzing a data sample corresponding to an integrated luminosity of $955 \mathrm{pb}^{-1}$. Only $t \bar{t}$ events, where one top quark decays semileptonically and the other hadronically are considered, leading to a signature of at least four jets, a charged lepton, and missing transverse energy due to the neutrino. The charge asymmetry is measured in the observable $\Delta y \cdot Q_{l}$, the rapidity difference of the semileptonically and hadronically decaying top quark times the charge of the charged lepton. The asymmetry is then given by the difference in the number of events with positive and negative $\Delta y \cdot Q_{l}$ values divided by the sum.

In preparation of this measurement, I conducted several Monte Carlo based studies concerning the asymmetry. As described in chapter 1.3 the charge asymmetry is caused by the interference of different matrix element amplitudes for the same final state, e.g. $t \bar{t}, t \bar{t} g$.... The harder the additional gluon, the more negative is the interference contribution of initial state (ISR) and final state gluon radiation (FSR) amplitudes. Overall a slightly positive value of about $5 \%$ is expected. Using the $t \bar{t}$ signal sample generated with $\mathrm{MC} @ \mathrm{NLO}$, the expected inclusive asymmetry value $A_{\text {inc }}$ is well reproduced. Leading order Monte Carlo generators like PyThiA and HERWIG lead, as expected, to an inclusive asymmetry of zero.

A Monte Carlo based study of the asymmetry at jet level has shown, that the value of the asymmetry depends on the number of reconstructed jets. In detail, we observe a linear dependence of the asymmetry on the number of jets, where the slope is negative. This jet bin effect is the result of a growing contribution of hard ISR and FSR interference. A similar linear dependence of the asymmetry on the number of jets is observed for leading-order parton showering Monte Carlos like PythiA and HERWIG. Here, the ISR and FSR interference is partly modeled by the angular ordering of gluon radiation implemented in the parton showering.

These findings motivated further studies concerning the effect of gluon radiation on the rapidity distribution of the produced top quarks. Thereby, we observe a certain dependence of the rapidity of the top quark on the strength and direction of the 
radiation. The higher the transverse momentum and the more central the radiation, the more negative becomes the mean of the rapidity distribution of the produced top quarks. This behavior explains the observed shift of the inclusive asymmetry value in the negative direction after applying our event selection cuts, which was the original motivation for the Monte Carlo based studies.

Due to these results, I performed the measurement in the inclusive data sample, as well as in the four- and five-jets sub-samples exclusively in order to be sensitive to the negative contribution of the asymmetry expected to occur in the five-jets subsample. I used a matrix method to correct the background subtracted event numbers for event selection and reconstruction effects. Because the matrix obtained from a PythiA signal sample $\left(A_{i n c}=0 \%\right)$ and obtained from a MC@NLO signal sample $\left(A_{i n c}=5 \%\right)$ are very similar, the correction method is proven to be independent of the inclusive asymmetry as long as $A_{i n c}$ is relatively close to the standard model prediction. Using the PYTHIA correction matrix, I obtain the following results:

$$
\begin{gathered}
A_{\text {inc }}\left(\Delta y \cdot Q_{l}\right)=0.23 \pm 0.12(\text { stat }) \pm \underset{0.057}{0.056}(\text { sys }) \\
A_{4}\left(\Delta y \cdot Q_{l}\right)=0.11 \pm 0.14(\text { stat }) \pm \underset{0.036}{0.036}(\text { sys }) \\
A_{5}\left(\Delta y \cdot Q_{l}\right)=0.37 \pm 0.30(\text { stat }) \pm \underset{0.066}{0.075}(\text { sys })
\end{gathered}
$$

The value measured in the four-jets bin is comparable to the expected slightly positive value of the charge asymmetry of about 5\%. Although we would have expected a negative asymmetry in the five-jets bin, the measured large positive value is not very significant due to low statistics. Since the measured asymmetry in the inclusive data sample reflects the measurement in the exclusive samples, it is higher than expected. The exclusive as well as the inclusive measurements are still statistically limited.

The main focus of this thesis was to understand the results of the performed MC studies of the asymmetry in top-pair production, and therefore to reach an appropriate interpretation of a measurement result. The studies revealed, that a measurement in exclusive jet bins offers the possibility to study the different contributions to the asymmetry. To optimize this attempt, we need to estimate the composition of the corresponding event sample, i.e. how many $t \bar{t}, t \bar{t} j, t \bar{t} j j, \ldots$ events contribute to an asymmetry measured in the respective jet bin. Due to the interpretation approach yielded by this thesis, a more precise analysis can be performed to understand better the origin of the charge asymmetry in top-pair production. 


\section{Appendix A}

\section{Statistical Uncertainty of Corrected Event Numbers}

Given are the efficiencies $\epsilon_{p}$ and $\epsilon_{n}$ with the corresponding binomial uncertainties $\sigma_{\epsilon_{p}}$ and $\sigma_{\epsilon_{n}}$, and the elements of the smearing matrix $S$ :

$$
S_{p p}=\frac{N_{p p}}{N_{p}^{s e l}}, \quad S_{p n}=\frac{N_{p n}}{N_{p}^{s e l}}, \quad S_{n p}=\frac{N_{n p}}{N_{n}^{s e l}}, \quad S_{p n}=\frac{N_{n n}}{N_{n}^{s e l}},
$$

The uncertainty of the elements $S_{i k}$ are described by the binomial error:

$$
\begin{aligned}
\sigma_{S_{p p}} & =\frac{1}{N_{p}^{s e l}} \sqrt{N_{p}^{s e l} \cdot S_{p p} \cdot\left(1-S_{p p}\right)} \\
\sigma_{S_{p n}} & =\frac{1}{N_{p}^{s e l}} \sqrt{N_{p}^{s e l} \cdot S_{p n} \cdot\left(1-S_{p n}\right)} \\
\sigma_{S_{n p}} & =\frac{1}{N_{n}^{s e l}} \sqrt{N_{n}^{s e l} \cdot S_{n p} \cdot\left(1-S_{n p}\right)} \\
\sigma_{S_{n n}} & =\frac{1}{N_{n}^{s e l}} \sqrt{N_{n}^{s e l} \cdot S_{n n} \cdot\left(1-S_{n n}\right)}
\end{aligned}
$$

The correction matrix $C$ connects smearing and efficiency:

$$
C=\left(\begin{array}{cc}
\epsilon_{p} & 0 \\
0 & \epsilon_{n}
\end{array}\right) \cdot\left(\begin{array}{cc}
S_{p p} & S_{n p} \\
S_{p n} & S_{n n}
\end{array}\right)=\left(\begin{array}{cc}
\epsilon_{p} \cdot S_{p p} & \epsilon_{p} \cdot S_{n p} \\
\epsilon_{n} \cdot S_{p n} & \epsilon_{n} \cdot S_{n n}
\end{array}\right)
$$

The error of the matrix elements of the correction matrix are calculated by Gaussian error propagation:

$$
\begin{aligned}
\sigma_{C_{p p}} & =\sqrt{\epsilon_{p}^{2} \cdot \sigma_{S_{p p}}+S_{p p}^{2} \cdot \sigma_{\epsilon_{p}}} \\
\sigma_{C_{p n}} & =\sqrt{\epsilon_{p}^{2} \cdot \sigma_{S_{p n}}+S_{p n}^{2} \cdot \sigma_{\epsilon_{p}}} \\
\sigma_{C_{n p}} & =\sqrt{\epsilon_{n}^{2} \cdot \sigma_{S_{n p}}+S_{n p}^{2} \cdot \sigma_{\epsilon_{n}}} \\
\sigma_{C_{n n}} & =\sqrt{\epsilon_{n}^{2} \cdot \sigma_{S_{n n}}+S_{n n}^{2} \cdot \sigma_{\epsilon_{n}}}
\end{aligned}
$$


Inverting the correction matrix yields:

$$
C^{-1}=\frac{1}{C_{p p} \cdot C_{n n}-C_{n p} \cdot C_{p n}} \cdot\left(\begin{array}{cc}
C_{n n} & -C_{n p} \\
-C_{p n} & C_{p p}
\end{array}\right)
$$

We introduce the abbreviation $X=1 /\left(C_{p p} \cdot C_{n n}-C_{n p} \cdot C_{p n}\right)$, whose statistical uncertainty $\sigma_{X}$ is given by:

$$
\sigma_{X}=\sqrt{C_{n n}^{2} \cdot \sigma_{C_{p p}}^{2}+C_{p n}^{2} \cdot \sigma_{C_{n p}}^{2}+C_{n p}^{2} \cdot \sigma_{C_{p n}}^{2}+C_{p p}^{2} \cdot \sigma_{C_{n n}}^{2}}
$$

The uncertainty of the inverted matrix elements is again derived from Gaussian error propagation:

$$
\begin{aligned}
\sigma_{C_{p p}^{-1}} & =\sqrt{\left(\frac{-C_{n n}}{X^{2}}\right)^{2} \cdot \sigma_{X}^{2}+\left(\frac{1}{X}\right)^{2} \cdot \sigma_{C_{n n}}^{2}} \\
\sigma_{C_{p n}^{-1}} & =\sqrt{\left(\frac{-C_{p n}}{X^{2}}\right)^{2} \cdot \sigma_{X}^{2}+\left(\frac{1}{X}\right)^{2} \cdot \sigma_{C_{p n}}^{2}} \\
\sigma_{C_{n p}^{-1}} & =\sqrt{\left(\frac{-C_{n p}}{X^{2}}\right)^{2} \cdot \sigma_{X}^{2}+\left(\frac{1}{X}\right)^{2} \cdot \sigma_{C_{n p}}^{2}} \\
\sigma_{C_{n n}^{-1}} & =\sqrt{\left(\frac{-C_{p p}}{X^{2}}\right)^{2} \cdot \sigma_{X}^{2}+\left(\frac{1}{X}\right)^{2} \cdot \sigma_{C_{p p}}^{2}}
\end{aligned}
$$

The true event numbers are calculated on the base of the inverted matrix from the background subtracted event numbers:

$$
\begin{gathered}
\hat{N}_{p}=C_{p p}^{-1} \cdot N_{p}^{B g S u b}+C_{n p}^{-1} \cdot N_{n}^{B g S u b} \\
\hat{N}_{n}=C_{p n}^{-1} \cdot N_{p}^{B g S u b}+C_{n n}^{-1} \cdot N_{n}^{B g S u b}
\end{gathered}
$$

whereby the uncertainty of the background subtracted numbers is given as:

$$
\begin{gathered}
\sigma_{N_{p}^{\text {BgSub }}}=\sqrt{N_{p}+N_{p}^{B g}} \\
\sigma_{N_{n}^{\text {BgSub }}}=\sqrt{N_{n}+N_{n}^{B g}}
\end{gathered}
$$

Finally, the statistical uncertainty of the corrected event numbers $\hat{N}_{p}$ and $\hat{N}_{n}$, which then enters the uncertainty of the asymmetry, can be obtained from Gaussian error propagation:

$$
\begin{aligned}
\sigma_{\hat{N}_{p}} & =\sqrt{\left(N_{p}^{B g S u b}\right)^{2} \cdot \sigma_{C_{p p}^{-1}}^{2}+\left(N_{n}^{B g S u b}\right)^{2} \cdot \sigma_{C_{n p}^{-1}}^{2}+\left(C_{p p}^{-1}\right)^{2} \cdot \sigma_{N_{p}^{B g S u b}}^{2}+\left(C_{n p}^{-1}\right)^{2} \cdot \sigma_{N_{n}^{B g S u b}}^{2}} \\
\sigma_{\hat{N}_{n}} & =\sqrt{\left(N_{p}^{B g S u b}\right)^{2} \cdot \sigma_{C_{p n}^{-1}}^{2}+\left(N_{n}^{B g S u b}\right)^{2} \cdot \sigma_{C_{n n}^{-1}}^{2}+\left(C_{p n}^{-1}\right)^{2} \cdot \sigma_{N_{p}^{B g S u b}}^{2}+\left(C_{n n}^{-1}\right)^{2} \cdot \sigma_{N_{n}^{B g S u b}}^{2}}
\end{aligned}
$$




\section{Bibliography}

[1] CDF Collaboration, F. Abe et al., Phys. Rev. Lett. 74, 2626 (1995).

[2] DØ Collaboration, S. Abachi et al., Phys. Rev. Lett. 74, 2632 (1995).

[3] S.L. Glashow, Nucl. Phys. 22, 579 (1961).

[4] S. Weinberg, Phys. Rev. Lett. 19, 1264 (1967).

[5] W.-M. Yao et al., J. Phys. G 33, 1 (2006).

[6] N. Cabbibo, Phys. Rev. Lett. 10, 531 (1963).

[7] M. Kobayashi and T. Maskawa, Prog. Theor. Phys. 49, 652 (1973).

[8] D.J. Griffiths, "Introduction to Elementary Particles", New York: Wiley, 1987.

[9] P.W. Higgs, Phys. Rev. Lett. 13, 508 (1964).

[10] T.E.W. Group (2006), hep-ex/0603039.

[11] M. Cacciari et al., J. High Energy Phys. 0404, 068 (2004).

[12] W. Wagner, Rep. Prog. Phys. 68, 2409-2494 (2005).

[13] H. L. Lai et al., Eur. Phys. G 33, 1 (2000).

[14] J.H. Kühn and G.Rodrigo, Phys. Rev. Lett. 81, 49 (1998).

[15] J.H. Kühn and G.Rodrigo, Phys. Rev. D59, 054017 (1999).

[16] D. Hirschbühl, "Measurement of the Charge Asymmetry and the $W$ Boson Helicity in Top-Antitop Quark Events with the CDF II Experiment", FERMILAB-THESIS-2005-80 (2005).

[17] P.H. Garbinicus, hep-ex/0406013.

[18] J. Farrington, " $H^{-}$Ion Beam Characterization", Fermilab Summer 2002 SIST Program.

[19] R.F. Welton, "Overview of High-Brightness $H^{-}$Ion Sources", Proceedings of LINAC 2002, Korea. 
[20] CDF II Collaboration, FERMILAB-PUB-96/390-E (1996).

[21] C.S. Hill et al., Nucl. Instrum. Meth. A 511, 118 (2003).

[22] A. Sill et al., Nucl. Instrum. Meth. A 447, 1 (2000).

[23] A. Afforder et al., Nucl. Instrum. Meth. A 453, 84 (2000).

[24] T. Affolder et al., Nucl. Instrum. Meth. A 526, 249 (2004).

[25] L. Balka et al., Nucl. Instrum. Meth. A 267, 272 (1988).

[26] S. Bertolucci et al., Nucl. Instrum. Meth. A 267, 301 (1988).

[27] M.G. Albrow et al., Nucl. Instrum. Meth. A 480, 524 (2002).

[28] A. Artikov et al., Nucl. Instrum. Meth. A 538, 358 (2000).

[29] M.Albrow, "CDF Run II Trigger Table and Dataset plan", CDF Internal Note No. 4718.

[30] B. Ashmanskas et al., Nucl. Instrum. Meth. A 518, 532 (2004).

[31] G. Gomez-Ceballos et al., Nucl. Instrum. Meth. A 518, 522 (2004).

[32] W. Badgett, H. Frisch, R.St. Denis, T. Vaiciulis, "The Good Run List", Internal CDF Note No. 5613.

[33] E. Sexton-Kennedy and P. Murat, eConf C0303241 (2003) THJT001, physics/0306112.

[34] Good Run List V13

http://www-cdf.fnal.gov/internal/dqm/goodrun/v13/goodv13.html.

[35] R. Erbacher et al., "Event Selection and t-tbar Signal Acceptance of the Winter 2005 Top Lepton + Jets Sample", Internal CDF Note No 7372.

[36] C. Hill, J. Incandela, and C. Mills, "Electron Identification in Offline Release 5.3", Internal CDF Note No. 7309.

[37] V. Martin, "High $p_{T}$ muons, recommended cuts and efficiencies for release 5.3.1", Internal CDF Note No. 7031.

[38] M. Tecchio, A. Varganov, B. Jayatilaka, D. Amidei, Y. Takeuchi, R. Tsuchiya, D. Whitson, and V. Boisvert, "Top Dilepton Cross Section Measurement using the GEN5 DIL Selection", Internal CDF Note No 7851.

[39] B. Flaugher, J. Müeller, "A Guide to JETCLU: The CDF Jet Cluster Algorithm", Internal CDF Note No. 1814.

[40] Generic Jet Corrections for Run II, Internal CDF Note No. 7358. 
[41] C. Rott et al., "SecVtx Optimization for the 2003 Winter Conferences", Internal CDF Note No. 6242.

[42] T. Chwalek, D. Hirschbühl, T. Müller, J. Wagner, W. Wagner, "Improvement of the Full Reconstruction of Top Anti-Top Pairs in Lepton+Jets Events", Internal CDF Note No. 8338.

[43] D. Acosta et al., "Introduction to Run II Jet Probability Heavy Flavor Tagger", Internal CDF Note No. 6315.

[44] T. Chwalek, "Measurement of the $W$ Boson Helicity in Top-Antitop Quark Events with the CDF II Experiment" (2006), FERMILAB-MASTERS-200604 (2006).

[45] M. Feindt, S.Richter, and W. Wagner, "A Neural Network $b$ Tagger for SingleTop Analyses", Internal CDF Note No. 7816.

[46] G. Altarelli, G. Parisi, "Asymptotic Freedom in Parton Language", Nucl. Phys. B 126 (1977) 298.

[47] T. Sjöstrand et al., Comput. Phys. Commun. 135, 238 (2001).

[48] HERWIG 6.5, G. Corcella, I.G. Knowles, G. Marchesini, S. Moretti, K. Odagiri, P. Richardson, M.H. Seymour, and B.R. Webber, hep-ph/0011363, hep$\mathrm{ph} / 0210213$.

[49] S. Frixione, P. Nason, and B.R. Webber, "Matching NLO QCD and parton showers in heavy flavour production", hep-ph/0305252. P. Richardson, M.H. Seymour and B.R. Webber, hep-ph/0011363, hep-ph/0210213.

[50] J. Collins, "Subtraction method for NLO corrections in Monte Carlo event generators for leptonproduction", JHEP 0005, 004 (2000), hep-ph/0001040.

[51] M.L. Mangano, M.Moretti, F. Piccinini, R. Pittau, and A. Polesa, "ALPGEN, a generator for hard multiparton processes in hadronic collisions", hep$\mathrm{ph} / 0206293$.

[52] F. Maltoni and T. Stelzer, hep-ph/0208156.

[53] T. Stelzer and W.F. Long, hep-ph/9401258.

[54] R. Brun and F. Carminati, CERN Programming Library Long Writeup W5013 (1993).

[55] Y. Kemp, "Identification of Electrons in the Forward Region of the CDF Experiment for the Search for Electroweak Top Quark Production", IEKP-KA/20061 (2006).

[56] D. Acosta et al., Phys. Rev. D71, 012005 (2005). 
[57] S.Catani, Y. L. Dokshitzer, M. H. Seymour, and B. R. Webber, Nucl. Phys. B406, 187 (1993).

[58] Talk of Tatsuya Masabuchi, Top Properties Meeting 07/07/06.

[59] A.D. Martin, R.G. Roberts, W.J. Stirling, and R.S. Thorne, Eur. Phys. J., C 4, 463 (1998).

[60] H.L. Lai et al., Eur. Phys. J., C 12, 375 (2000). 


\section{Danksagung}

An erster Stelle möchte ich mich bei Herrn Prof. Dr. Th. Müller für die Möglichkeit bedanken, in seiner Top-Quark-Arbeitsgruppe diese Diplomarbeit durchzuführen, und auch dafür, dass ich die Ergebnisse meiner Arbeit während eines zweiwöchigen Aufenthalts am Fermilab vorstellen durfte.

Ebenso bedanke ich mich bei Herrn Priv.-Doz. Dr. Wolfgang Wagner für die Übernahme des Korreferats meiner Arbeit und die sehr engagierte Betreuung.

Meiner Betreuerin Dr. Jeannine Wagner danke ich für die sehr gute Betreuung der Arbeit und ihren unermüdlichen Einsatz bei der Ergründung der unerwarteten Aspekte der Analyse. Besonders danke ich ihr für das sehr aufmerksame Korrekturlesen dieser Arbeit und die daraus resultierten Verbesserungen.

Dr. Dominic Hirschbühl, der sich meiner Betreuung vor Ort angenommen hat, möchte ich besonders danken für die geduldige Unterstützung sowie sein Engagement bei der Suche nach dem Schlüssel zur Asymmetrie. Deweiteren danke ich ihm für das Korrekturlesen dieser Arbeit und die abwechslungsreichen zwei Wochen in Chicago.

Auch Thorsten Chwalek danke ich für das Korrekturlesen der Arbeit und für die vielen interessanten Diskussionen während unserer angenehmen gemeinsamen Bürozeit. Svenja Richter, Adonis Papaikonomou, Jan Lück und Matthias Bühler für das freundschaftliche Arbeitsklima und jegliche Hilfestellungen während meiner Arbeit. Ganz besonders möchte ich mich bei denen bedanken, die mit ihren Diskussionsbeiträgen und Rechercheaktivitäten zur Entwicklung eines umfassenden Verständnisses der theoretischen Grundlagen meiner Arbeit beigetragen haben. Mein Dank gilt allen Mitgliedern des EKP im 8. und 9. Stock, die durch ihr Engagement, besonders das des Admin-Teams und des Sekretariats, und ihre offene Art ein sehr angenehmes, professionelles Arbeitsumfeld schaffen. Dr. Yves Kemp danke ich für seine schnelle und unkomplizierte Hilfe bei diversen Computerproblemen.

Meinem Freund Benny danke ich für seine Liebe und sein Verständnis und dafür, dass er mein Leben um so vieles bereichert. Ich bedanke mich bei Stefanie Elbracht und Britta Wawerzinek für die tolle Freundschaft seit unseres gemeinsamen Studienbeginns und die gegenseitige Unterstützung, ohne die die Physik bestimmt nicht zu zähmen gewesen wäre. Meinen Großeltern und Verwandten, die meine Wünsche und Pläne immer respektiert haben, danke ich für all ihre Unterstützung. Ein Dank geht auch an meinem Bruder für unser vertrauensvolles gutes Verhältnis, das mir sehr viel bedeutet.

Abschließend möchte ich mich ganz besonders bei meinen Eltern bedanken, zum einen, dass sie mir dieses Ziel finanziell ermöglicht haben, aber viel mehr dafür, dass 
sie mir die Freiheit gaben, meinen Weg zu gehen und dabei nie an mir gezweifelt haben. Dass nun auch das letzte Stück dieses Weges geschafft ist, verdanke ich vor allem meiner Mutter. Sie hat mir trotz ihres zähen Kampfes gegen $\mathrm{MH}$ während der letzten Jahre immer den Rücken frei gehalten. Ihre Stärke und Ausdauer wird mir stets ein Vorbild sein.

Hiermit versichere ich, die vorliegende Arbeit selbständig verfasst und nur die angegebenen Hilfsmittel verwendet zu haben.

Julia Weinelt

Karlsruhe, 15. Dezember 2006 